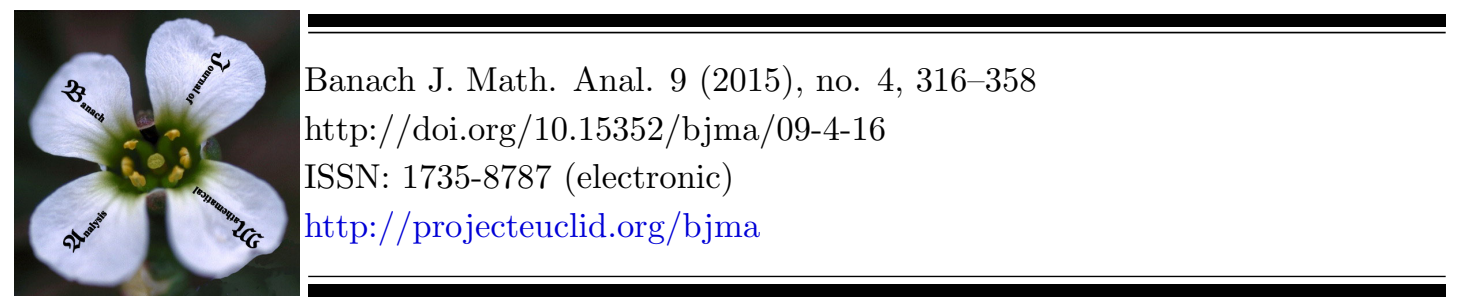

\title{
CROSSED PRODUCTS OF ALGEBRAS OF UNBOUNDED OPERATORS
}

\author{
MARIA FRAGOULOPOULOU ${ }^{1 *}$, ATSUSHI INOUE ${ }^{2}$ AND KLAUS-DETLEF KÜRSTEN ${ }^{3}$ \\ Communicated by M. Joiţa
}

\begin{abstract}
Consider a closed $O^{*}$-algebra $\mathcal{M}$ on a dense linear subspace $\mathcal{D}$ of a Hilbert space $\mathcal{H}$, a locally compact group $G$ with left invariant Haar measure $d s$ and an action $\alpha$ of $G$ on $\mathcal{M}$. Under some natural conditions, the $O^{*}-$ crossed product $\mathcal{M} \underset{\alpha}{\stackrel{O^{*}}{\rtimes}} G$ of $\mathcal{M}$ and the $G W^{*}$-crossed product $\mathcal{M} \underset{\alpha}{G W^{*}} G$ are introduced. When $G$ is also abelian, the dual action $\widehat{\alpha}$ of the dual group $\widehat{G}$ on $\mathcal{M}{ }_{\rtimes}^{O^{*}} G$ and on $\mathcal{M} \stackrel{G W^{*}}{\rtimes} G$ is defined, which makes it possible to study the crossed products

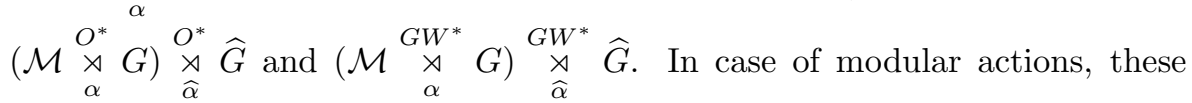
constructions are used to obtain results on duality of type II-like and type III-like $G W^{*}$-algebras.
\end{abstract}

\section{INTRODUCTION AND PRELIMINARIES}

1.1. Introduction. Research papers on crossed products exist in the literature since 1958; see, for instance, [31, 34]. Though, a strong impetus to the development of the theory of crossed products was given by the thesis of A. Connes (1973) [5] and the paper of M. Takesaki (1973) [28] "Duality for crossed products and the structure of von Neumann algebras of type III". A crossed product in the theory of von Neumann algebras is a method of construction of a new von Neumann algebra from a given one on which a group acts. There are various forms

Date: Received: Jan. 12, 2015; Accepted: Feb. 21, 2015.

* Corresponding author.

2010 Mathematics Subject Classification. Primary 47L65, secondary 47L60, 46L55, 46L60.

Key words and phrases. $O^{*}$-covariant system, $O^{*}$-crossed product, duality of $G W^{*}$-crossed products, modular action. 
and generalizations of crossed products and their construction is considered as one of the most significant ideas in the theory of operator algebras not only for the theory itself, but also for the applications. Recall that in quantum physics the observables are represented by non-commuting operators and in some physical models the observables are assumed to form a $C^{*}$-algebra. So time evolution of the observables is described by a $C^{*}$-dynamical system $[4,7,14,18,19,30]$. Since observables are often modeled by unbounded operators, dynamical systems based on unbounded operator algebras or even on more general systems of unbounded operators occur in a natural way, see [3, 16]. The interplay between operator algebras and dynamical systems is realized through crossed products. The latter offer very powerful tools in various studies connected with operator algebras and their strong link with dynamical systems relates them with quantum and statistical mechanics. Moreover, the construction of crossed products gave rise to the first non-trivial examples of factors and besides reduced the study of the purely infinite algebras to the study of semifinite algebras, which is much more familiar. For all these "Tomita-Takesaki Modular Theory" and its applications to mathematical physics (see $[13,24,26]$ ) has played a fundamental role.

All the above gave us the motivation for the study of "crossed products of algebras of unbounded operators", provided that the yeast has already existed (see e.g., $[1,8,9,10,11,12,13]$ ). Our study is mainly connected with the results presented in the monograph of A. Van Daele [32] on "Continuous crossed products and type III von Neumann algebras" (see also [18, Chapter 7], [24, Chapter IV], [29, Chapter X]). There is a rich literature on "classical" crossed products, in general, and apart from the monograph [32] and the chapters on crossed products cited before, various books have been published on the algebraic or topological aspects of the subject; see, for example, [14, 15, 17, 33]. Crossed products of unbounded operator algebras by group actions appeared in the article of $\mathrm{Yu}$. Savchuk and K. Schmüdgen [20], Section 4. Unbounded crossed products by actions of quantum groups appeared even earlier in the literature (see [22, 23]). In [20] there was used an approach different to the one presented here and it could be an interesting question of further investigation, whether crossed products treated there could adapted to our setting of crossed products of generalized von Neumann algebras (for short, $G W^{*}$-algebras).

The structure of the present paper is as follows: In the preliminary Subsection 1.2, some basic background material concerning $O^{*}$-algebras, $G W^{*}$-algebras, traces, and tensor products is collected.

In Section 2, $O^{*}$-crossed products $\mathcal{M}^{O^{*}} G$ are defined and investigated. Here $\mathcal{M}$ stands for a closed $O^{*}$-algebra with domain $\mathcal{D}, G$ is a locally compact group with left invariant Haar measure $d s$ and $\alpha$ is an action of $G$ on $\mathcal{M}$. Denoting by $\mathcal{H}$ the Hilbert space completion of $\mathcal{D}$, there will be considered also the Hilbert space $L^{2}(G, \mathcal{H})$ completion of the linear space $\mathcal{C}_{c}(G, \mathcal{H})$ of all continuous $\mathcal{H}$-valued functions on $G$ with compact support, with respect to the inner product

$$
(\xi \mid \eta):=\int_{G}(\xi(s) \mid \eta(s)) d s
$$


The triple $(\mathcal{M}, G, \alpha)$ is said to be an $O^{*}$-covariant system, whenever

$$
\begin{aligned}
& D_{0}(\mathcal{M}, G, \alpha)=\left\{\xi \in \mathcal{C}_{c}(G, \mathcal{H}) ; \xi(s) \in \mathcal{D}, \text { for all } s \in G\right. \text { and the map } \\
&\left.\quad G \ni s \mapsto \alpha_{s^{-1}}(x) \xi(s) \in \mathcal{H} \text { is continuous, for all } x \in \mathcal{M}\right\}
\end{aligned}
$$

is dense in $L^{2}(G, \mathcal{H})$. In this case, the $O^{*}$-crossed products $\mathcal{M} \underset{\alpha}{O_{\alpha}^{*}} G$ of $\mathcal{M}$ by the action $\alpha$ of $G$ is defined to be the closure of the $O^{*}$-algebra generated by operators $A$ with domain $D_{0}(\mathcal{M}, G, \alpha)$ defined by

$$
(A \xi)(s)=\alpha_{s^{-1} t}(x) \xi\left(t s^{-1}\right)
$$

where $x \in \mathcal{M}$ and $t \in G$ (Definition 2.7).

In Section 3, using the results of Section 2 and in particular the domain $D(\mathcal{M}, G, \alpha)$ of the $O^{*}$-crossed product, we introduce the $G W^{*}$-crossed product. Assuming that $(\mathcal{M}, G, \alpha)$ is an $O^{*}$-covariant system such that $\mathcal{M}_{w}^{\prime} \mathcal{D} \subset \mathcal{D}$, where $\mathcal{M}_{w}^{\prime}$ is the weak commutant of $\mathcal{M}$, and that the action $\alpha$ is spatial and strongly continuous, the $G W^{*}$-crossed product $\mathcal{M} \underset{\alpha}{G W^{*}} G$ of $\mathcal{M}$ by $\alpha$ is defined to be the space of elements of $\mathcal{L}^{\dagger}(D(\mathcal{M}, G, \alpha))$, which are affiliated to the von

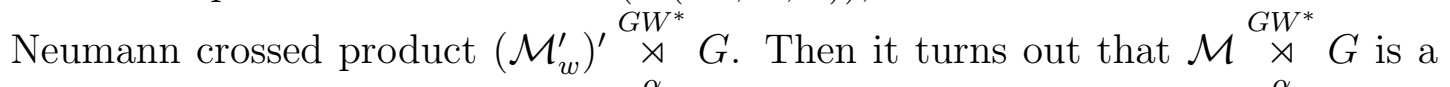
$G W^{*}$-algebra on $D(\mathcal{M}, G, \alpha)$ over $\left(\mathcal{M}_{w}^{\prime}\right)^{\prime} \underset{\alpha}{G W^{*}} G$ containing $\mathcal{M} \underset{\alpha}{\underset{\sim}{O^{*}}} G$ (Theorem 3.5). A characterization of a crossed product as an algebra of fixed points for a certain action of $G$ on a tensor product algebra, which is known for von Neumann algebras, will be generalized to $G W^{*}$-crossed products (Proposition 3.6).

In Section 4 under the additional assumption that $G$ is abelian, there will be

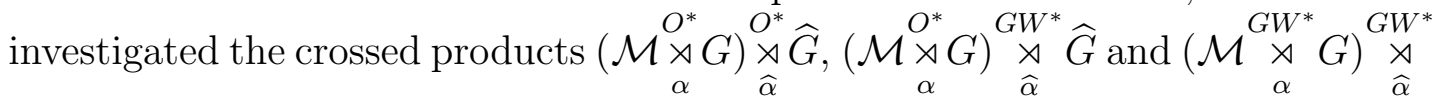
$\widehat{G}$ by a dual action $\widehat{\alpha}$ of the dual group $\widehat{G}$ on $\underset{\mathcal{M}}{\mathcal{O}_{\alpha}^{*}} G$ and $\mathcal{M}_{\alpha} \underset{\alpha}{G W^{*}} G$, respectively. It is proved that $\widehat{\alpha}$ is spacial on both $\mathcal{M} \underset{\alpha}{O^{*}} G$ and $\mathcal{M} \underset{\alpha}{\stackrel{G}{\rtimes}} G$ and strongly continuous on $\mathcal{M} \underset{\alpha}{O_{\alpha}^{*}} G$ (Lemma 4.3). For a complete characterization of $\left(\mathcal{M}_{\alpha}^{\mathcal{O}_{\alpha}^{*}} G\right) \underset{\widehat{\alpha}}{\rtimes} \widehat{O^{*}} \widehat{G}$ see Theorems 4.12 and 4.13. For $\left(\mathcal{M} \underset{\alpha}{O^{*}} G\right) \underset{\widehat{\alpha}}{\stackrel{G W^{*}}{\rtimes}} \widehat{G}$ and $\left(\mathcal{M}_{\underset{\alpha}{\rtimes}}^{G W^{*}} G\right) \underset{\widehat{\alpha}}{\stackrel{G W^{*}}{\rtimes}} \widehat{G}$, we show that if the domain $D(\mathcal{M}, G, \alpha))$ is invariant under the weak commutant of $\mathcal{M} \underset{\alpha}{O_{\alpha}^{*}} G$

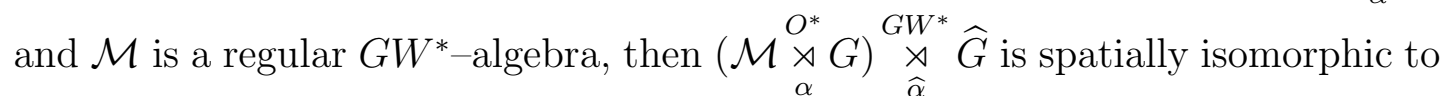
a specific $G W^{*}$-algebra and isomorphic to a $G W^{*}$-tensor product defined by the von Neumann algebras $\left(\mathcal{M}_{w}^{\prime}\right)^{\prime}, \mathcal{B}\left(L^{2}(G)\right)$ and $W D(\mathcal{M}, G, \alpha)$ (Proposition 4.14).

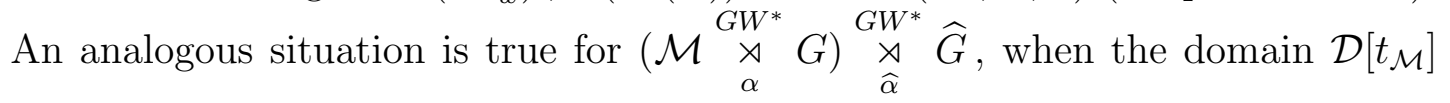


is a Fréchet locally convex space; in this particular case, $\left(\mathcal{M} \underset{\alpha}{\underset{\rtimes}{O^{*}} G}\right) \underset{\widehat{\alpha}}{\stackrel{G W^{*}}{\rtimes}} \widehat{G}$ and $\left(\mathcal{M} \underset{\alpha}{G W^{*}} G\right) \underset{\widehat{\alpha}}{G W^{*}} \widehat{G}$ coincide whenever $\mathcal{M}$ is regular (Theorem 4.16).

The subjects of the final Section 5 are crossed products by modular actions. Given an $O^{*}$-algebra $\mathcal{M}$ on $\mathcal{D}$ in $\mathcal{H}$ such that $\mathcal{M}_{w}^{\prime} \mathcal{D} \subset \mathcal{D}$, the notion of a standard vector $\xi_{0}$ for $\mathcal{M}$ is defined in such a way that the modular operator $\Delta_{\xi_{0}}$ of the left Hilbert algebra $\left(\mathcal{M}_{w}^{\prime}\right)^{\prime} \xi_{0}$ exists in the sense of [27, 29] and that the formula $\sigma_{t}(x):=\Delta_{\xi_{0}}^{i t} x \Delta_{\xi_{0}}^{-i t}(x \in \mathcal{M}$ and $t \in \mathbb{R})$ defines a 1-parameter group of *-automorphisms of $\mathcal{M}$, called the modular action associated with the standard vector $\xi_{0}$. Then it turns out that $(\mathcal{M}, \mathbb{R}, \sigma)$ is an $O^{*}$-covariant system and the crossed products $\mathcal{M} \underset{\sigma}{\underset{O^{*}}{x}} \mathbb{R}$ and $\mathcal{M} \underset{\sigma}{\underset{\rtimes}{\rtimes}} \mathbb{R}$ are well-defined. By using the generalized Connes cocycle theorem [13, Theorem 2.5.6], it will be shown, that these crossed products as well as $(\mathcal{M} \underset{\sigma}{\stackrel{G}{\rtimes}} \mathbb{R}) \underset{\widehat{\sigma}}{\underset{\sim}{\rtimes}} \mathbb{R}$ are independent of the special choice of the standard vector $\xi_{0}$ up to a unitary equivalences (Propositions 5.3, 5.4). Furthermore, there will be defined a faithful normal trace $\tau$ on $\mathcal{M}_{\underset{\sigma}{\rtimes}}^{G W^{*}} G\left(\sigma=\sigma^{\xi_{0}}\right.$, depending on the standard vector $\xi_{0}$ ). Under some additional conditions, $\tau$ is also semifinite (Theorem 5.9). All constructions will be illustrated by an example. Finally, these constructions are used to obtain results on duality of type II-like $G W^{*}$-algebras and type III-like $G W^{*}$-algebras (Theorem 5.14).

1.2. Preliminaries. Let $\mathcal{D}$ be a dense subspace of a complex Hilbert space $\mathcal{H}$ with inner product denoted by $(\mid)$. Denote by $\mathcal{B}(\mathcal{H})$ the $*$-algebra of all bounded linear operators on $\mathcal{H}$ and by $\mathcal{L}(\mathcal{D})$ the algebra of all linear operators from $\mathcal{D}$ to $\mathcal{D}$. Let

$$
\mathcal{L}^{\dagger}(\mathcal{D}):=\left\{x \in \mathcal{L}(\mathcal{D}) ; \mathcal{D} \subset \mathcal{D}\left(x^{*}\right) \text { and } x^{*} \mathcal{D} \subset \mathcal{D}\right\}
$$

where $\mathcal{D}\left(x^{*}\right)$ means domain of $x^{*}$. Then $\mathcal{L}^{\dagger}(\mathcal{D})$ is a $*$-algebra under the usual algebraic operations and the involution defined by $x \longmapsto x^{\dagger}:=x^{*}\left\lceil_{\mathcal{D}}\right.$. A $*-$ subalgebra $\mathcal{M}$ of $\mathcal{L}^{\dagger}(\mathcal{D})$ that contains the identity operator of $\mathcal{D}$ as an element is called an $O^{*}$-algebra on $\mathcal{D}$ in $\mathcal{H}$. Given an $O^{*}$-algebra as before, $\mathcal{D}$ is endowed with the graph topology $t_{\mathcal{M}}$ given by the seminorms $\left\{\|\cdot\|_{x}: x \in \mathcal{M}\right\}$ (see $[13,21]$ ), with $\|\xi\|_{x}=\|x \xi\|, \xi \in \mathcal{D}$. Then $\mathcal{M}$ is called closed if the locally convex space $\mathcal{D}\left[t_{\mathcal{M}}\right]$ is complete. Denote by $\widetilde{\mathcal{D}}(\mathcal{M})$ the completion of $\mathcal{D}\left[t_{\mathcal{M}}\right]$. Then $\widetilde{\mathcal{D}}(\mathcal{M})=$ $\bigcap_{x \in \mathcal{M}} D(\bar{x})$, where $\bar{x}$ denotes the closure of the operator $x$. Put now $\widetilde{x}=\bar{x} \uparrow_{\widetilde{\mathcal{D}}(\mathcal{M})}$, $x \in \mathcal{M}$ and $\widetilde{\mathcal{M}}=\{\widetilde{x} ; x \in \mathcal{M}\} . \widetilde{\mathcal{M}}$ is then a closed $O^{*}$-algebra on $\widetilde{\mathcal{D}}(\mathcal{M})$ in $\mathcal{H}$, which is called the closure of $\mathcal{M}$. We say that $\mathcal{M}$ is closed, if $\mathcal{D}=\widetilde{\mathcal{D}}(\mathcal{M})$. If $\mathcal{D}$ coincides with $\mathcal{D}^{*}(\mathcal{M}):=\bigcap_{x \in \mathcal{M}} D\left(x^{*}\right), \mathcal{M}$ is said to be self-adjoint.

We define now the notion of a " $G W^{*}$-algebra", which is an unbounded generalization of a von Neumann algebra. Let $\mathcal{L}^{\dagger}(\mathcal{D}, \mathcal{H})$ be the set of all linear operators $x$ from $\mathcal{D}$ to $\mathcal{H}$ such that $\mathcal{D} \subset \mathcal{D}\left(x^{*}\right)$. Then $\mathcal{L}^{\dagger}(\mathcal{D}, \mathcal{H})$ is a $\dagger$-invariant vector space under the usual operations $x+y, \lambda x$ and the involution $x^{\dagger}:=x^{*} \uparrow \mathcal{D}$, for any $x, y \in \mathcal{L}^{\dagger}(\mathcal{D}, \mathcal{H})$ and $\lambda \in \mathbb{C}$. A $\dagger$-invariant subspace of $\mathcal{L}^{\dagger}(\mathcal{D}, \mathcal{H})$ is called an $O^{*}$-vector space on $\mathcal{D}$. Among other locally convex topologies on $\mathcal{L}^{\dagger}(\mathcal{D}, \mathcal{H})$ 
are the so-called strong and strong* topologies, which will be denoted by $\tau_{s}, \tau_{s^{*}}$, respectively. These topologies are defined by the families of seminorms

$$
p_{\xi}(x):=\|x \xi\|, p_{\xi}^{*}(x):=p_{\xi}(x)+p_{\xi}\left(x^{\dagger}\right) \text { resp., } \forall \xi \in \mathcal{D} \text { and } \forall x \in \mathcal{L}^{\dagger}(\mathcal{D}, \mathcal{H}) \text {. }
$$

The induced strong or strong* topology on an $O^{*}$-vector space $\mathcal{M}$ is called strong or strong* topology on $\mathcal{M}$. Let $\mathcal{M}$ be an $O^{*}$-vector space on $\mathcal{D}$. We define the commutants $\mathcal{M}_{w}^{\prime}$ and $\mathcal{M}_{c}^{\prime}$ of $\mathcal{M}$ as follows:

$$
\begin{gathered}
\mathcal{M}_{w}^{\prime}:=\left\{c \in \mathcal{B}(\mathcal{H}) ;(c x \xi \mid \eta)=\left(c \xi \mid x^{\dagger} \eta\right), \forall x \in \mathcal{M} \text { and } \xi, \eta \in \mathcal{D}\right\}, \\
\mathcal{M}_{c}^{\prime}:=\left\{s \in \mathcal{L}^{\dagger}(\mathcal{D}) ; s x=x s, \forall x \in \mathcal{M}\right\} .
\end{gathered}
$$

Then $\mathcal{M}_{w}^{\prime}$ is a $*$-invariant subspace of $\mathcal{B}(\mathcal{H})$, but it is not necessarily an algebra; $\mathcal{M}_{c}^{\prime}$ is an $O^{*}$-algebra on $\mathcal{D}$. Suppose that $\mathcal{M}$ is self-adjoint. Then it follows that $\mathcal{M}_{w}^{\prime} \mathcal{D} \subset \mathcal{D}$, which implies that $\mathcal{M}_{w}^{\prime}$ is a von Neumann algebra and $\bar{x} \eta\left(\mathcal{M}_{w}^{\prime}\right)^{\prime}$, for every $x \in \mathcal{M}$. Recall that $\bar{x} \eta\left(\mathcal{M}_{w}^{\prime}\right)^{\prime}$ means that the operator $\bar{x}$ is affiliated with the von Neumann algebra $\left(\mathcal{M}_{w}^{\prime}\right)^{\prime}$. Let $[\cdot]^{\tau_{s^{*}}}$ denote $\tau_{s^{*}}$-closure and let $\mathcal{M}$ be an $O^{*}$-algebra on $\mathcal{D}$ in $\mathcal{H}$. If there exists a von Neumann algebra $\mathcal{M}_{0}$ on $\mathcal{H}$ such that $\mathcal{M}_{0}^{\prime} \mathcal{D} \subset \mathcal{D}$ and $\mathcal{M}=\left[\mathcal{M}_{0}\lceil\mathcal{D}]^{\tau_{s^{*}}} \cap \mathcal{L}^{\dagger}(\mathcal{D})\right.$, then $\mathcal{M}$ is said to be a $G W^{*}$-algebra (abbreviation of the term "generalized $W^{*}$-algebra") on $\mathcal{D}$ over $\mathcal{M}_{0}$. It is well-known that

$$
\begin{gathered}
\mathcal{M} \text { is a } G W^{*} \text { - algebra over } \mathcal{M}_{0} \\
\Longleftrightarrow \mathcal{M}=\left(\mathcal{M}_{0}^{\prime} \uparrow \mathcal{D}\right)_{c}^{\prime}:=\left\{x \in \mathcal{L}^{\dagger}(\mathcal{D}) ; x c \xi=c x \xi, \forall c \in \mathcal{M}_{0}^{\prime} \text { and } \xi \in \mathcal{D}\right\} \\
\Longleftrightarrow \mathcal{M}=\left\{x \in \mathcal{L}^{\dagger}(\mathcal{D}) ; \bar{x} \eta \mathcal{M}_{0}\right\} .
\end{gathered}
$$

In particular, if $\mathcal{M}_{w}^{\prime} \mathcal{D} \subset \mathcal{D}$, then considering the unbounded bicommutant

$$
\mathcal{M}_{w c}^{\prime \prime}:=\left(\mathcal{M}_{w}^{\prime}\lceil\mathcal{D})_{c}^{\prime}=\left\{x \in \mathcal{L}^{\dagger}(\mathcal{D}) ; x c \xi=c x \xi, \forall c \in \mathcal{M}_{w}^{\prime} \text { and } \xi \in \mathcal{D}\right\},\right.
$$

one has the following (in this regard, see also [13, Proposition 1.7.5])

$$
\mathcal{M}_{w c}^{\prime \prime}=\left[\left(\mathcal{M}_{w}^{\prime}\right)^{\prime}\lceil\mathcal{D}]^{\tau_{s}^{*}} \cap \mathcal{L}^{\dagger}(\mathcal{D})=\left\{x \in \mathcal{L}^{\dagger}(\mathcal{D}) ; \bar{x} \eta\left(\mathcal{M}_{w}^{\prime}\right)^{\prime}\right\} .\right.
$$

Then $\mathcal{M}_{w c}^{\prime \prime}$ is a $G W^{*}$-algebra over $\left(\mathcal{M}_{w}^{\prime}\right)^{\prime}$; in this case, we shall simply say that $\mathcal{M}_{w c}^{\prime \prime}$ is a $G W^{*}$-algebra. A closed $O^{*-a l g e b r a} \mathcal{M}$ on $\mathcal{D}$ is said to be a $G W^{*}$-algebra if $\mathcal{M}_{w}^{\prime} \mathcal{D} \subset \mathcal{D}$ and $\mathcal{M}=\mathcal{M}_{w c}^{\prime \prime}$. We remark that when $\mathcal{M}$ is a $G W^{*}$-algebra over $\mathcal{M}_{0}, \mathcal{M}_{0} \neq\left(\mathcal{M}_{w}^{\prime}\right)^{\prime}$, in general; therefore $\mathcal{M}$ is not necessarily a $G W^{*}$-algebra. We introduce now the notion of "regularity" for $G W^{*}$-algebras. A $G W^{*}$-algebra $\mathcal{M}$ on $\mathcal{D}$ is said to be regular if $\left(\overline{\mathcal{M}_{b}}\right)^{\prime \prime}=\left(\mathcal{M}_{w}^{\prime}\right)^{\prime}$, where $\mathcal{M}_{b}:=\{a \in \mathcal{M} ; \bar{a} \in \mathcal{B}(\mathcal{H})\}$, corresponds to the bounded part of $\mathcal{M}$ and $\overline{\mathcal{M}_{b}}:=\left\{\bar{a} ; a \in \mathcal{M}_{b}\right\}$. A $G W^{*}$-algebra $\mathcal{M}$ on $\mathcal{D}$ over $\mathcal{M}_{0}$ is called regular if $\left(\overline{\mathcal{M}_{b}}\right)^{\prime \prime}=\mathcal{M}_{0}$. Clearly, if $\mathcal{M}$ is a regular $G W^{*}$-algebra, then it is a regular $G W^{*}$-algebra over $\left(\mathcal{M}_{w}^{\prime}\right)^{\prime}$. Furthermore, it is easily shown that if $\mathcal{M}$ is a regular $G W^{*}$-algebra over $\mathcal{M}_{0}$, then $\left(\overline{\mathcal{M}_{b}}\right)^{\prime \prime}=\mathcal{M}_{0}=$ $\left(\mathcal{M}_{w}^{\prime}\right)^{\prime}$, so that $\mathcal{M}$ is a regular $G W^{*}$-algebra too.

Now, let $\mathcal{A}$ be a unital $*$-algebra and $\mathcal{D}$ a dense subspace of a Hilbert space $\mathcal{H}$. A $*$-representation $\pi$ of $\mathcal{A}$ on $\mathcal{D}$ is a homomorphism of $\mathcal{A}$ onto an $O^{*}$-algebra $\mathcal{M}$ on $\mathcal{D}$ in $\mathcal{H}$, such that $\pi\left(a^{*}\right)=\pi(a)^{\dagger}$, for every $a \in \mathcal{A}$ and $\pi(1)=\mathbb{I}$, where 1 is the unit of $\mathcal{A}$ and $\mathbb{I}$ is the identity operator in $\mathcal{M}$. We shall say that $\pi$ is faithful, if $\pi(a)=0, a \in \mathcal{A}$, implies $a=0$. Given a $*$-representation $\pi$ of $\mathcal{A}$ on $\mathcal{D}$, we shall write, for distinction, $\mathcal{D}(\pi)$ instead of just $\mathcal{D}$. Denote by $t_{\pi}$ the graph 
topology $t_{\pi(\mathcal{A})}$ on $\mathcal{D}(\pi)$ with respect to the $O^{*}$-algebra $\mathcal{M}=\pi(\mathcal{A})$. Whenever $\mathcal{D}(\pi)\left[t_{\pi}\right]$ is complete, $\pi$ will be called closed.

The normality of a $*$-representation of an $O^{*}$-algebra is defined as follows: The positive wedge $\mathcal{M}_{+}$of an $O^{*}$-algebra $\mathcal{M}$ on $\mathcal{D}$ is defined by

$$
\mathcal{M}_{+}=\{x \in \mathcal{M} ;(x \xi \mid \xi) \geq 0, \forall \xi \in \mathcal{D}\} .
$$

We say that a net $\left\{x_{i}\right\}$ in $\mathcal{M}_{+}$converges increasingly to an element $x$ of $\mathcal{M}_{+}$ and write $x_{i} \uparrow x$ if $\left(x_{i} \xi \mid \xi\right) \leqq\left(x_{j} \xi \mid \xi\right)$, for each $\xi \in \mathcal{D}$, whenever $i \leqq j$ and $\lim _{i}\left(x_{i} \xi \mid \xi\right)=(x \xi \mid \xi)$, for each $\xi \in \mathcal{D}$. A $*$-representation $\pi$ of $\mathcal{M}$ is said to be normal if $\pi\left(x_{i}\right) \uparrow \pi(x)$, whenever $x_{i} \uparrow x$; cf. [18].

If $\mathcal{M}$ is an $O^{*}$-algebra on $\mathcal{D}$ in $\mathcal{H}$, the symbol $A u t(\mathcal{M})$ will stand for the set of all $*$-automorphisms of $\mathcal{M}$. An element $\alpha \in A u t(\mathcal{M})$ will be called spacial [21, p. 167], if there is an isometry $u$ of $\mathcal{H}$ onto itself such that $u \mathcal{D}=\mathcal{D}$ and $\alpha(x) \xi=u x u^{-1} \xi$, for any $x \in \mathcal{M}$ and $\xi \in \mathcal{D}$. A $*$-automorphism $\alpha$ from $\mathcal{M}$ onto $\mathcal{M}$ will be called strongly continuous, if it is continuous as a map from $\mathcal{M}\left[\tau_{s}\right]$ onto $\mathcal{M}\left[\tau_{s}\right]$. A trace on an $O^{*}$-algebra $\mathcal{M}$ is a map

$\tau: \mathcal{M}_{+} \longrightarrow[0, \infty]: x \longmapsto \tau(x)$ with the following properties:

(1) $\tau(x+y)=\tau(x)+\tau(y), \forall x, y \in \mathcal{M}_{+}$;

(2) $\tau(\lambda x)=\lambda \tau(x), \forall \lambda \geq 0$ and $x \in \mathcal{M}_{+}$, where $0 \cdot(+\infty)=0$;

(3) $\tau\left(x^{\dagger} x\right)=\tau\left(x x^{\dagger}\right), \forall x \in \mathcal{M}$,

cf. $[2,13]$.

A trace $\tau$ is said to be faithful if $\tau\left(x^{\dagger} x\right)=0, x \in \mathcal{M}$, implies $x=0$. Yet, $\tau$ is called normal if for every net $\left\{x_{\lambda}\right\}$ in $\mathcal{M}_{+}$such that $x_{\lambda} \uparrow x, x \in \mathcal{M}_{+}$, one has $\tau\left(x_{\lambda}\right) \uparrow \tau(x)$. Finally, we shall say that $\tau$ is semifinite, whenever $\mathfrak{N}_{\tau}$ is strongly dense in $\mathcal{M}$, where

$$
\mathfrak{N}_{\tau}:=\left\{x \in \mathcal{M} ; \tau\left((a x)^{\dagger}(a x)\right)<\infty, \quad \forall a \in \mathcal{M}\right\} .
$$

Further, suppose that $\mathcal{H}_{1}$ and $\mathcal{H}_{2}$ are Hilbert spaces. Their algebraic tensor product $\mathcal{H}_{1} \otimes \mathcal{H}_{2}$ is a pre-Hilbert space under the inner product defined by

$$
\left(\xi_{1} \otimes \xi_{2} \mid \eta_{1} \otimes \eta_{2}\right)=\left(\xi_{1} \mid \eta_{1}\right)\left(\xi_{2} \mid \eta_{2}\right), \xi_{1}, \eta_{1} \in \mathcal{H}_{1} \text { and } \xi_{2}, \eta_{2} \in \mathcal{H}_{2} .
$$

The completion of $\mathcal{H}_{1} \otimes \mathcal{H}_{2}$ under the norm induced by the preceding inner product is a Hilbert space called the Hilbert space tensor product of $\mathcal{H}_{1}$ and $\mathcal{H}_{2}$ and is denoted by $\mathcal{H}_{1} \bar{\otimes} \mathcal{H}_{2}$. Let $\mathcal{D}_{1}$ and $\mathcal{D}_{2}$ be dense subspaces of $\mathcal{H}_{1}$ and $\mathcal{H}_{2}$, respectively. Then the algebraic tensor product $\mathcal{D}_{1} \otimes \mathcal{D}_{2}$ is a dense subspace in $\mathcal{H}_{1} \bar{\otimes} \mathcal{H}_{2}$.

Let $x_{1}$ and $x_{2}$ be linear operators on $\mathcal{D}_{1}$ and $\mathcal{D}_{2}$, respectively. The algebraic tensor product $x_{1} \otimes x_{2}$ of $x_{1}, x_{2}$ on $\mathcal{D}_{1} \otimes \mathcal{D}_{2}$ is defined by

$$
\left(x_{1} \otimes x_{2}\right)\left(\xi_{1} \otimes \xi_{2}\right)=x_{1} \xi_{1} \otimes x_{2} \xi_{2}, \xi_{1} \in \mathcal{D}_{1}, \xi_{2} \in \mathcal{D}_{2} .
$$

In particular, if $x_{1}$ and $x_{2}$ are bounded, then $x_{1} \otimes x_{2}$ has a continuous extension to $\mathcal{H}_{1} \bar{\otimes} \mathcal{H}_{2}$, which is called tensor product of $x_{1}$ and $x_{2}$ and it is denoted by $x_{1} \bar{\otimes} x_{2}$. If $\mathcal{M}_{1}, \mathcal{M}_{2}$ are von Neumann algebras on $\mathcal{H}_{1}, \mathcal{H}_{2}$ respectively, then the von Neumann algebra on $\mathcal{H}_{1} \bar{\otimes} \mathcal{H}_{2}$ generated by $\left\{x_{1} \bar{\otimes} x_{2} ; x_{1} \in \mathcal{M}_{1}, x_{2} \in \mathcal{M}_{2}\right\}$ is called the von Neumann (or $W^{*_{-}}$) tensor product of $\mathcal{M}_{1}$ and $\mathcal{M}_{2}$ and is denoted 
by $\mathcal{M}_{1}{ }^{W^{*}} \otimes \mathcal{M}_{2}$. In order to define unbounded tensor products generated by $\mathcal{M}_{1}$ and $\mathcal{M}_{2}$ (see Section 3, discussion after the proof of Theorem 3.5), we make use of the preceding $W^{*}$-tensor product $\mathcal{M}_{1} \stackrel{W^{*}}{\otimes} \mathcal{M}_{2}$ of $\mathcal{M}_{1}$ and $\mathcal{M}_{2}$.

Suppose now that $\mathcal{M}_{1}$ and $\mathcal{M}_{2}$ are $O^{*}$-algebras on $\mathcal{D}_{1}$ and $\mathcal{D}_{2}$, respectively. Then

$$
\mathcal{M}_{1} \otimes \mathcal{M}_{2}:=\left\{\sum_{k=1}^{n} x_{1}^{(k)} \otimes x_{2}^{(k)} ; x_{1}^{(k)} \in \mathcal{M}_{1}, x_{2}^{(k)} \in \mathcal{M}_{2}\right\}
$$

is an $O^{*}$-algebra on $\mathcal{D}_{1} \otimes \mathcal{D}_{2}$, whose closure is called the tensor product of $\mathcal{M}_{1}$ and $\mathcal{M}_{2}$ and is denoted by $\mathcal{M}_{1} \widetilde{\otimes} \mathcal{M}_{2}$. The domain $\mathcal{D}\left(\mathcal{M}_{1} \widetilde{\otimes} \mathcal{M}_{2}\right)$ of the closed $O^{*}$-algebra $\mathcal{M}_{1} \widetilde{\otimes} \mathcal{M}_{2}$ is denoted by $\mathcal{D}_{1} \widetilde{\otimes} \mathcal{D}_{2} ;$ namely,

$$
\mathcal{D}_{1} \widetilde{\otimes} \mathcal{D}_{2}=\bigcap_{x \in \mathcal{M}_{1} \otimes \mathcal{M}_{2}} \mathcal{D}(\bar{x}) .
$$

For $x_{1} \in \mathcal{M}_{1}$ and $x_{2} \in \mathcal{M}_{2}, \overline{x_{1} \otimes x_{2}} \uparrow_{\mathcal{D}_{1} \widetilde{\otimes} \mathcal{D}_{2}}$ is denoted by $x_{1} \widetilde{\otimes} x_{2}$ and

$$
\mathcal{M}_{1} \widetilde{\otimes} \mathcal{M}_{2}:=\left\{\sum_{k=1}^{n} x_{1, k} \widetilde{\otimes} x_{2, k} ; x_{1, k} \in \mathcal{M}_{1}, x_{2, k} \in \mathcal{M}_{2}\right\} .
$$

If $\mathcal{M}_{1}$ and $\mathcal{M}_{2}$ are bounded $O^{*}$-algebras, then $\mathcal{D}_{1} \widetilde{\otimes} \mathcal{D}_{2}=\mathcal{H}_{1} \bar{\otimes} \mathcal{H}_{2}$ and $x_{1} \widetilde{\otimes} x_{2}=$ $x_{1} \bar{\otimes} x_{2}$, for any $x_{1} \in \mathcal{M}_{1}, x_{2} \in \mathcal{M}_{2}$. In this case $\mathcal{M}_{1} \widetilde{\otimes} \mathcal{M}_{2}$ is the linear span of the operators $\left\{x_{1} \bar{\otimes} x_{2} ; x_{1} \in \mathcal{M}_{1}, x_{2} \in \mathcal{M}_{2}\right\}$ and $\left(\mathcal{M}_{1} \widetilde{\otimes} \mathcal{M}_{2}\right)^{\prime \prime}$ coincides with the $W^{*}$-tensor product $\left(\overline{\mathcal{M}_{1}}\right)^{\prime \prime} W^{*} \otimes\left(\overline{\mathcal{M}_{2}}\right)^{\prime \prime}$ of the von Neumann algebras $\left(\overline{\mathcal{M}_{1}}\right)^{\prime \prime}$ and $\left(\overline{\mathcal{M}_{2}}\right)^{\prime \prime}$.

Let now $G$ be a locally compact group equipped with a left invariant Haar measure $d s$ and let $\mathcal{H}$ be a Hilbert space. Denote by $\mathcal{C}_{c}(G, \mathcal{H})$ the set of all continuous maps from $G$ into $\mathcal{H}$ with compact support and by $L^{2}(G)$ the Hilbert space of all (equivalence classes of) square integrable functions from $G$ into $\mathbb{C}$ with respect to $d s$. Under the inner product

$$
(\xi \mid \eta)=\int_{G}(\xi(s) \mid \eta(s)) d s
$$

$\mathcal{C}_{c}(G, \mathcal{H})$ becomes a pre-Hilbert space, whose completion, under the norm induced by the foregoing inner product, is a Hilbert space denoted by $L^{2}(G, \mathcal{H})$. The Hilbert space $\mathcal{H} \bar{\otimes} L^{2}(G)$ can be canonically identified with $L^{2}(G, \mathcal{H})$ through the isomorphism $U$, given by

$$
\left(U\left(\xi_{0} \otimes f\right)\right)(s)=f(s) \xi_{0}, \quad s \in G,
$$

for all $\xi_{0} \in \mathcal{H}$ and $f \in \mathcal{C}_{c}(G)$, the set of all $\mathbb{C}$-valued continuous functions on $G$ with compact support [32, Proposition 2.2].

\section{2. $\mathrm{O}^{*}$ - CROSSED PRODUCTS}

In this section we define a crossed product of an $O^{*}$-algebra by an action of a locally compact group. Throughout the whole section $\mathcal{M}$ will be a closed $O^{*}$ algebra on a dense subspace $\mathcal{D}$ in a Hilbert space $\mathcal{H}, G$ a locally compact group with Haar measure $d s$ and $\alpha$ an action of $G$ on $\mathcal{M}$, that is, $G \ni s \mapsto \alpha_{s} \in$ Aut $\mathcal{M}$ 
is a homomorphism. We remark that $\alpha$ is not necessarily strongly continuous since the modular action we deal with in Section 5 is not necessarily strongly continuous.

Consider now the pre-Hilbert space $\mathcal{C}_{c}(G, \mathcal{H})$, whose completion is the Hilbert space $L^{2}(G, \mathcal{H})$. We shall define an $O^{*}$-crossed product by the action $\alpha$ of $G$. Let

$$
\begin{aligned}
& D_{0}(\mathcal{M}, G, \alpha)=\left\{\xi \in \mathcal{C}_{c}(G, \mathcal{H}) ; \xi(s) \in \mathcal{D}, \quad \forall s \in G\right. \text { and } \\
&\left.\quad G \ni s \mapsto \alpha_{s^{-1}}(x) \xi(s) \in \mathcal{H} \text { is continuous, } \forall x \in \mathcal{M}\right\} .
\end{aligned}
$$

Definition 2.1. The triple $(\mathcal{M}, G, \alpha)$ is called an $O^{*}$-covariant system if the linear space $D_{0}(\mathcal{M}, G, \alpha)$ is dense in the Hilbert space $L^{2}(G, \mathcal{H})$.

Lemma 2.2. Suppose that

$$
\mathcal{C}_{\alpha}(\mathcal{D}):=\left\{\xi_{0} \in \mathcal{D} ; G \ni s \mapsto \alpha_{s^{-1}}(x) \xi_{0} \in \mathcal{H} \text { is continuous, } \forall x \in \mathcal{M}\right\}
$$

is dense in $\mathcal{H}$. Then $(\mathcal{M}, G, \alpha)$ is an $O^{*}$-covariant system.

Proof. It is clear that

$\mathcal{C}_{\alpha}(\mathcal{D}) \otimes \mathcal{C}_{c}(G):=$ linear span of $\left\{\xi_{0} \otimes f ; \xi_{0} \in \mathcal{C}_{\alpha}(\mathcal{D}), f \in \mathcal{C}_{c}(G)\right\} \subset D_{0}(\mathcal{M}, G, \alpha)$, where $\left(\xi_{0} \otimes f\right)(s):=f(s) \xi_{0}, s \in G$. Furthermore, since $\mathcal{C}_{\alpha}(\mathcal{D})$ is dense in $\mathcal{H}$, it follows that $\mathcal{C}_{\alpha}(\mathcal{D}) \otimes \mathcal{C}_{c}(G)$ is dense in $L^{2}(G, \mathcal{H})$, which implies that $(\mathcal{M}, G, \alpha)$ is an $O^{*}$-covariant system.

Given an $O^{*}$-covariant system $(\mathcal{M}, G, \alpha)$ and $x \in \mathcal{M}$, put

$$
\left(\pi_{\alpha}^{0}(x) \xi\right)(s)=\alpha_{s^{-1}}(x) \xi(s), \quad s \in G, \quad \xi \in D_{0}(\mathcal{M}, G, \alpha) .
$$

In this regard, we have

Lemma 2.3. Suppose that $(\mathcal{M}, G, \alpha)$ is an $O^{*}$-covariant system. Then $\pi_{\alpha}^{0}$ is a *-representation of $\mathcal{M}$ on $D_{0}(\mathcal{M}, G, \alpha)$. Moreover, if $\mathcal{C}_{\alpha}(\mathcal{D})$ is dense in $\mathcal{H}$, then $\pi_{\alpha}^{0}$ is a faithful $*$-representation.

Proof. For all $x, y \in \mathcal{M}$ and $\xi, \eta \in D_{0}(\mathcal{M}, G, \alpha)$ we have

$$
\begin{aligned}
\alpha_{s^{-1}}(x)\left(\pi_{\alpha}^{0}(y) \xi\right)(s) & =\alpha_{s^{-1}}(x y) \xi(s)=\left(\pi_{\alpha}^{0}(x y) \xi\right)(s), \quad \forall s \in G, \text { and } \\
\left(\pi_{\alpha}^{0}(x) \xi \mid \eta\right) & =\int_{G}\left(\alpha_{s^{-1}}(x) \xi(s) \mid \eta(s)\right) d s \\
& =\int_{G}\left(\xi(s) \mid \alpha_{s}^{-1}\left(x^{\dagger}\right) \eta(s)\right) d s=\left(\xi \mid \pi_{\alpha}^{0}\left(x^{\dagger}\right) \eta\right),
\end{aligned}
$$

which implies that $\pi_{\alpha}^{0}$ is a $*-$ representation of $\mathcal{M}$ on $D_{0}(\mathcal{M}, G, \alpha)$. Suppose that $\mathcal{C}_{\alpha}(\mathcal{D})$ is dense in $\mathcal{H}$. We show that $\pi_{\alpha}^{0}$ is faithful. Indeed, suppose $\pi_{\alpha}^{0}(x)=0$. Then since

$$
\left\|\pi_{\alpha}^{0}(x)\left(\xi_{0} \otimes f\right)\right\|^{2}=\int_{G}|f(s)|^{2}\left\|\alpha_{s^{-1}}(x) \xi_{0}\right\|^{2} d s=0,
$$

for any $\xi_{0} \in \mathcal{C}_{\alpha}(\mathcal{D})$ and $f \in \mathcal{C}_{c}(G)$ and $G \ni s \mapsto\left\|\alpha_{s^{-1}}(x) \xi_{0}\right\| \in \mathbb{R}$ is continuous, it follows that $\alpha_{s^{-1}}(x) \xi_{0}=0$, for each $s \in G$. In particular, $x \xi_{0}=0$, for each $\xi_{0} \in \mathcal{C}_{\alpha}(\mathcal{D})$. Hence, $x=0$. 
Definition 2.4. The action $\alpha$ is said to be spatial if there exists a strongly continuous unitary representation $w$ of $G$ on $\mathcal{H}$ such that $w_{s} \mathcal{D} \subset \mathcal{D}$ and $\alpha_{s}(x)=$ $w_{s} x w_{s}^{*}$, for all $s \in G$ and $x \in \mathcal{M}$.

Lemma 2.5. Suppose that $\alpha$ is spatial. Then $(\mathcal{M}, G, \alpha)$ is an $O^{*}$-covariant system and $\pi_{\alpha}^{0}$ is a faithful normal *-representation of $\mathcal{M}$ on $D_{0}(\mathcal{M}, G, \alpha)$.

Proof. Since $\alpha$ is spatial, there exists a strongly continuous unitary representation $w$ of $G$ on $\mathcal{H}$ such that $w_{s} \mathcal{D} \subset \mathcal{D}$ and $\alpha_{s}(x)=w_{s} x w_{s}^{*}$, for every $s \in G$ and $x \in \mathcal{M}$. Put now

$$
(W \xi)(s)=w_{s} \xi(s), \quad s \in G, \quad \xi \in L^{2}(G, \mathcal{H}) .
$$

Then $W$ is a unitary operator on $L^{2}(G, \mathcal{H})$ and $\left(W^{*} \xi\right)(s)=w_{s}^{*} \xi(s)$, for all $s \in G$ and $\xi \in L^{2}(G, \mathcal{H})$. Since

$$
\alpha_{s^{-1}}(x)\left(W^{*}\left(\xi_{0} \otimes f\right)\right)(s)=f(s) w_{s}^{*} x \xi_{0}, s \in G, x \in \mathcal{M}, f \in \mathcal{C}_{c}(G), \xi_{0} \in \mathcal{D},
$$

it follows that $W^{*}\left(\mathcal{D} \otimes \mathcal{C}_{c}(G)\right) \subset D_{0}(\mathcal{M}, G, \alpha)$, which implies that $(\mathcal{M}, G, \alpha)$ is an $O^{*}$-covariant system. By Lemma $2.3, \pi_{\alpha}^{0}$ is a $*$-representation of $\mathcal{M}$ on $D_{0}(\mathcal{M}, G, \alpha)$. Furthermore, by $(2.1)$, we have

$$
\left\|\pi_{\alpha}^{0}(x) W^{*}\left(\xi_{0} \otimes f\right)\right\|^{2}=\left\|x \xi_{0}\right\|^{2} \int_{G}|f(s)|^{2} d s,
$$

for all $x \in \mathcal{M}, \xi_{0} \in \mathcal{D}$ and $f \in \mathcal{C}_{c}(G)$, which implies that $\pi_{\alpha}^{0}$ is faithful. We show now that $\pi_{\alpha}^{0}$ is normal.

Indeed, let $\left\{x_{i}\right\}$ in $\mathcal{M}_{+}$with $x_{i} \uparrow x \in \mathcal{M}_{+}$. Then for any $\xi \in D_{0}(\mathcal{M}, G, \alpha)$ we have

$$
\begin{aligned}
0 \leqq\left(\alpha_{s^{-1}}\left(x_{i}\right) \xi(s) \mid \xi(s)\right) & =\left(x_{i} w_{s} \xi(s) \mid w_{s} \xi(s)\right) \uparrow\left(x w_{s} \xi(s) \mid w_{s} \xi(s)\right) \\
& =\left(\alpha_{s^{-1}}(x) \xi(s) \mid \xi(s)\right)
\end{aligned}
$$

for each $s \in G$, which by the Beppo-Levi theorem implies that

$$
\left(\pi_{\alpha}^{0}\left(x_{i}\right) \xi \mid \xi\right) \uparrow\left(\pi_{\alpha}^{0}(x) \xi \mid \xi\right) .
$$

Hence, $\pi_{\alpha}^{0}$ is normal and this completes the proof.

For any $t \in G$ we define a linear operator $\lambda(t)$ on $L^{2}(G, \mathcal{H})$ by

$$
(\lambda(t) \xi)(s)=\xi\left(t^{-1} s\right), \quad \xi \in L^{2}(G, \mathcal{H}), s \in G .
$$

Then we have the following

Lemma 2.6. $\lambda$ is a strongly continuous unitary representation of $G$ on $L^{2}(G, \mathcal{H})$ such that

$$
\begin{gathered}
\lambda(t) \uparrow D_{0}(\mathcal{M}, G, \alpha) \in \mathcal{L}^{\dagger}\left(D_{0}(\mathcal{M}, G, \alpha)\right) \text { and } \\
\lambda(t) \pi_{\alpha}^{0}(x) \lambda(t)^{\dagger} \xi=\pi_{\alpha}^{0}\left(\alpha_{t}(x)\right) \xi, \quad t \in G, \quad x \in \mathcal{M}, \xi \in D_{0}(\mathcal{M}, G, \alpha) .
\end{gathered}
$$

Proof. It is easily shown that $\lambda$ is a strongly continuous unitary representation of $G$ on $L^{2}(G, \mathcal{H})$. Take an arbitrary $t \in G$. Since for all $x \in \mathcal{M}$ and $\xi \in$ $D_{0}(\mathcal{M}, G, \alpha)$

$$
\alpha_{s^{-1}}(x)(\lambda(t) \xi)(s)=\alpha_{\left(t^{-1} s\right)^{-1}}\left(\alpha_{t^{-1}}(x)\right) \xi\left(t^{-1} s\right) \quad \text { and } \lambda(t)^{*}=\lambda\left(t^{-1}\right), \quad s, t \in G,
$$


it follows that $\lambda(t)\left\lceil_{D_{0}(\mathcal{M}, G, \alpha)} \in \mathcal{L}^{\dagger}\left(D_{0}(\mathcal{M}, G, \alpha)\right)\right.$. Furthermore, we have

$$
\begin{aligned}
\left(\left(\lambda(t) \pi_{\alpha}^{0}(x) \lambda(t)^{\dagger}\right) \xi\right)(s) & =\left(\pi_{\alpha}^{0}(x) \lambda(t)^{\dagger}\right) \xi\left(t^{-1} s\right)=\alpha_{\left(t^{-1} s\right)^{-1}}(x)\left(\lambda(t)^{\dagger} \xi\right)\left(t^{-1} s\right) \\
& =\alpha_{s^{-1}}\left(\alpha_{t}(x)\right) \xi(s)=\left(\pi_{\alpha}^{0}\left(\alpha_{t}(x)\right) \xi\right)(s), \quad s \in G,
\end{aligned}
$$

for all $x \in \mathcal{M}$ and $\xi \in D_{0}(\mathcal{M}, G, \alpha)$. This completes the proof.

By Lemma 2.6 the linear span $\mathfrak{M}$ of the operators of the form $\pi_{\alpha}^{0}(x) \lambda(t)$ with $x \in \mathcal{M}$ and $t \in G$ is an $O^{*}$-algebra on $D_{0}(\mathcal{M}, G, \alpha)$ in $L^{2}(G, \mathcal{H})$.

Definition 2.7. We denote by $\mathcal{M}{ }^{O^{*}} G$ the closure of the $O^{*}$-algebra $\mathfrak{M}$, that is, $D(\mathcal{M}, G, \alpha):=\mathcal{D}\left(\mathcal{M} \underset{\alpha}{\underset{O^{*}}{\rtimes}} G\right)$ is the completion of $D_{0}(\mathcal{M}, G, \alpha)\left[t_{\mathfrak{M}}\right]$,

$$
\begin{aligned}
\mathcal{D}\left(\mathcal{M} \underset{\alpha}{{ }_{\rtimes}^{*}} G\right) & =\bigcap_{X \in \mathfrak{M}} \mathcal{D}(\bar{X}), \text { and } \\
\mathcal{M} \underset{\alpha}{\stackrel{O^{*}}{\rtimes}} G & =\{\bar{X} \uparrow D(\mathcal{M}, G, \alpha) ; X \in \mathfrak{M}\} .
\end{aligned}
$$

$\mathcal{M} \underset{\alpha}{O^{*}} G$ is called the $O^{*}-$ crossed product of $\mathcal{M}$ by the action $\alpha$ of $G$.

By Lemma 2.6 we have the following

Lemma 2.8. Let $\pi_{\alpha}$ be the closure of the $*$-representation $\pi_{\alpha}^{0}$ of $\mathcal{M}$. Then

$$
t_{\substack{\mathcal{M}_{\alpha}{ }_{\alpha}^{*} G \\ \text { (n) }}}=t_{\pi_{\alpha}} \text { and } D(\mathcal{M}, G, \alpha)=\mathcal{D}\left(\pi_{\alpha}\right),
$$

where in the first equality we mean the corresponding graph topologies.

We now consider the case where $\alpha$ is strongly continuous.

Proposition 2.9. Suppose that $\alpha$ is strongly continuous. Then the following statements hold:

$(1) \mathcal{C}_{\alpha}(\mathcal{D})=\mathcal{D}$. Hence, $(\mathcal{M}, G, \alpha)$ is always an $O^{*}$-covariant system and $\pi_{\alpha}$ is faithful.

(2) $\mathcal{D} \otimes \mathcal{C}_{c}(G)$ is dense in $D(\mathcal{M}, G, \alpha)$ with respect to the graph topology $t_{\mathcal{M}}^{O_{\alpha}^{*} G} \cdot$

Proof. (1) It is clear that $\mathcal{C}_{\alpha}(\mathcal{D})=\mathcal{D}$. By Lemma $2.3(\mathcal{M}, G, \alpha)$ is an $O^{*}$-covariant system and $\pi_{\alpha}$ is faithful.

(2) By (1) and Lemma 2.2, D $\otimes \mathcal{C}_{c}(G) \subset D_{0}(\mathcal{M}, G, \alpha)$. By Lemma 2.8, it suffices to show that $\mathcal{D} \otimes \mathcal{C}_{c}(G)$ is dense in $D_{0}(\mathcal{M}, G, \alpha)$ with respect to $t_{\pi_{\alpha}}$. Take an arbitrary $\zeta \in D_{0}(\mathcal{M}, G, \alpha)$ and any neighborhood $U_{\zeta}$ of $\zeta$ with respect to $t_{\pi_{\alpha}}$. Then there exist $\varepsilon>0$ and a finite subset $\left\{x_{0}:=\mathbb{I}, x_{1}, \ldots, x_{n}\right\}$ of $\mathcal{M}$ such that

$$
\left\{\xi \in D_{0}(\mathcal{M}, G, \alpha) ;\left\|\pi_{\alpha}\left(x_{k}\right) \xi-\pi_{\alpha}\left(x_{k}\right) \zeta\right\|<\varepsilon, k=0,1, \ldots, n\right\} \subset U_{\zeta} .
$$

Take an open subset $V$ of $G$ with finite Haar measure $|V|$, which contains $K$, the support of $\zeta$. Take an arbitrary $s_{0} \in K$. Since, for any $x \in \mathcal{M}$, the maps

$$
G \ni t \longmapsto \alpha_{t^{-1}}(x) \zeta\left(s_{0}\right) \in \mathcal{H} \text { and } G \ni t \longmapsto \alpha_{t^{-1}}(x) \zeta(t) \in \mathcal{H}
$$


are continuous, there exists a neighborhood $V_{s_{0}}$ of $s_{0}$ contained in $V$ such that

$$
\begin{gathered}
\left\|\alpha_{t^{-1}}\left(x_{k}\right) \zeta\left(s_{0}\right)-\alpha_{s_{0}^{-1}}\left(x_{k}\right) \zeta\left(s_{0}\right)\right\|<\frac{\varepsilon}{2|V|} \\
\left\|\alpha_{t^{-1}}\left(x_{k}\right) \zeta(t)-\alpha_{s_{0}^{-1}}\left(x_{k}\right) \zeta\left(s_{0}\right)\right\|<\frac{\varepsilon}{2|V|}
\end{gathered}
$$

for all $t \in V_{s_{0}}$ and $k=0,1, \ldots, n$. Then by the compactness of $K$ there exists a finite subset $\left\{s_{1}, s_{2}, \ldots, s_{m}\right\}$ of $K$ such that $K \subset \bigcup_{j=1}^{m} V_{s_{j}}$, consequently there exists a subset $\left\{h_{1}, h_{2}, \ldots, h_{m}\right\}$ of $\mathcal{C}_{c}(G)$ such that

- the support of $h_{j} \subset V_{s_{j}}, j=1,2, \ldots, m$;

- $0 \leqq \sum_{j=1}^{m} h_{j}(s) \leqq 1, \quad \forall s \in G$;

- $\sum_{j=1}^{m} h_{j}(s)=1, \quad \forall s \in K$.

Put $\xi=\sum_{j=1}^{m} h_{j} \zeta\left(s_{j}\right)$. Then $\xi \in \mathcal{D} \otimes \mathcal{C}_{c}(G)$. Let $s \in G$. Then $h_{j}(s)=0$, if $s \notin V_{s_{j}}$ and if $s \in V_{s_{j}}$, by (2.2) we obtain

$$
\begin{gathered}
\left\|\alpha_{s^{-1}}\left(x_{k}\right) \zeta\left(s_{j}\right)-\alpha_{s_{j}^{-1}}\left(x_{k}\right) \zeta\left(s_{j}\right)\right\|<\frac{\varepsilon}{2 \sqrt{|V|}}, \\
\left\|\alpha_{s^{-1}}\left(x_{k}\right) \zeta(s)-\alpha_{s_{j}^{-1}}\left(x_{k}\right) \zeta\left(s_{j}\right)\right\|<\frac{\varepsilon}{2 \sqrt{|V|}},
\end{gathered}
$$

which implies that

$$
\begin{aligned}
\| \alpha_{s^{-1}}\left(x_{k}\right) \xi(s) & -\alpha_{s^{-1}}\left(x_{k}\right) \zeta(s) \| \\
& =\left\|\sum_{j=1}^{m} h_{j}(s) \alpha_{s^{-1}}\left(x_{k}\right) \zeta\left(s_{j}\right)-\sum_{j=1}^{m} h_{j}(s) \alpha_{s^{-1}}\left(x_{k}\right) \zeta(s)\right\| \\
& \leqq \sum_{j=1}^{m} h_{j}(s)\left\|\alpha_{s^{-1}}\left(x_{k}\right) \zeta\left(s_{j}\right)-\alpha_{s^{-1}}\left(x_{k}\right) \zeta(s)\right\| \\
& \leqq \sum_{j=1}^{m} h_{j}(s)\left(\left\|\alpha_{s^{-1}}\left(x_{k}\right) \zeta\left(s_{j}\right)-\alpha_{s_{j}^{-1}}\left(x_{k}\right) \zeta\left(s_{j}\right)\right\|\right. \\
& \left.\quad+\left\|\alpha_{s_{j}^{-1}}\left(x_{k}\right) \zeta\left(s_{j}\right)-\alpha_{s^{-1}}\left(x_{k}\right) \zeta(s)\right\|\right) \\
& <\frac{\varepsilon}{\sqrt{|V|}}, \quad k=0,1, \ldots, n .
\end{aligned}
$$


So we conclude that

$$
\begin{aligned}
\left\|\pi_{\alpha}\left(x_{k}\right) \xi-\pi_{\alpha}\left(x_{k}\right) \zeta\right\|^{2} & =\int_{G}\left\|\alpha_{s^{-1}}\left(x_{k}\right) \xi(s)-\alpha_{s^{-1}}\left(x_{k}\right) \zeta(s)\right\|^{2} d s \\
& =\int_{V}\left\|\alpha_{s^{-1}}\left(x_{k}\right) \xi(s)-\alpha_{s^{-1}}\left(x_{k}\right) \zeta(s)\right\|^{2} d s \\
& <\frac{\varepsilon^{2}}{|V|} \cdot|V|=\varepsilon^{2}, \quad k=0,1, \ldots, n
\end{aligned}
$$

which means $\xi \in V_{\zeta}$. This completes the proof.

\section{3. $G W^{*}-$ CROSSED PRODUCTS}

Throughout this section $\mathcal{M}$ is a closed $O^{*}$-algebra on $\mathcal{D}$ in $\mathcal{H}$ such that $\mathcal{M}_{w}^{\prime} \mathcal{D} \subset$ $\mathcal{D}, G$ is a locally compact group with Haar measure $d s$ and $\alpha$ is an action of $G$ on $\mathcal{M}$, which is spatial; that is, $\alpha_{s}(x)=w_{s} x w_{s}^{*}, x \in \mathcal{M}, s \in G$, where $w$ is a strongly continuous unitary representation of $G$ on $\mathcal{H}$ such that $w_{s} \mathcal{D} \subset \mathcal{D}$, for each $s \in G$.

By Lemma $2.5,(M, G, \alpha)$ is an $O^{*}$-covariant system and $\pi_{\alpha}$ is a faithful closed *-representation of $\mathcal{M}$. Hence, the $O^{*}$-crossed product $\underset{\mathcal{M}}{\stackrel{O^{*}}{\rtimes}} G$ is well-defined. Furthermore, we put

$$
\alpha_{s}(a)=w_{s} a w_{s}^{*}, \quad a \in\left(\mathcal{M}_{w}^{\prime}\right)^{\prime}, \quad s \in G .
$$

Then $\alpha$ is a strongly continuous action of $G$ on the von Neumann algebra $\left(\mathcal{M}_{w}^{\prime}\right)^{\prime}$ and so we can consider the covariant system $\left(\left(\mathcal{M}_{w}^{\prime}\right)^{\prime}, \alpha, G\right)$. The crossed product of the von Neumann algebra $\left(\mathcal{M}_{w}^{\prime}\right)^{\prime}$ by the action $\alpha$ of $G$ will be denoted by $\left(\mathcal{M}_{w}^{\prime}\right)^{\prime} \rtimes G$. For any $a \in \mathcal{B}(\mathcal{H})$ we define a bounded linear operator $L_{a}$ on $L^{2}(G, \mathcal{H})$ by

$$
\left(L_{a} \xi\right)(s)=a \xi(s), \quad s \in G, \quad \xi \in L^{2}(G, \mathcal{H}) .
$$

For $t \in G$ we also define a unitary operator $\rho(t)$ on $L^{2}(G, \mathcal{H})$ by

$$
(\rho(t) \xi)(s)=\Delta(t)^{\frac{1}{2}} \xi(s t), \quad s \in G, \quad \xi \in L^{2}(G, \mathcal{H}),
$$

where $\Delta$ is the modular function of $G$. Then we have the following

Lemma 3.1. (1) $L_{a}=a \bar{\otimes} 1$, for every $a \in \mathcal{B}(\mathcal{H})$.

(2) For every $t \in G, \rho(t)=\mathbb{I} \bar{\otimes} \rho_{t}$, where $\left(\rho_{t} f\right)(s):=\Delta(t)^{\frac{1}{2}} f(s t), s \in G, f \in$ $L^{2}(G)$; II and 1 are the identity operators on $\mathcal{H}$ and $L^{2}(G)$, respectively.

Proof. The claims follow from the relations

$$
\begin{gathered}
\left((a \otimes 1)\left(\xi_{0} \otimes f\right)\right)(s)=\left(a \xi_{0} \otimes f\right)(s)=f(s) a \xi_{0}=\left(L_{a}\left(\xi_{0} \otimes f\right)\right)(s) \text { and } \\
\left(\left(I I \otimes \rho_{t}\right)\left(\xi_{0} \otimes f\right)\right)(s)=\left(\rho_{t} f\right)(s) \xi_{0}=\left(\rho(t)\left(\xi_{0} \otimes f\right)\right)(s),
\end{gathered}
$$

respectively, for all $\xi_{0} \in \mathcal{D}$ and $f \in \mathcal{C}_{c}(G)$.

By [32, Theorem 3.12] the commutant of $\left(\mathcal{M}_{w}^{\prime}\right)_{\alpha}^{\prime} \underset{\alpha}{ }$ is characterized by the following

Lemma 3.2. $\left(\left(\mathcal{M}_{w}^{\prime}\right)_{\alpha}^{\prime} \underset{\alpha}{ } G\right)^{\prime}=\left\{a^{\prime} \bar{\otimes} 1, w_{t} \bar{\otimes} \rho_{t} ; a^{\prime} \in \mathcal{M}_{w}^{\prime}, t \in G\right\}^{\prime \prime}$. 
Lemma 3.3. (1) For any $a^{\prime} \in \mathcal{M}_{w}^{\prime}, x \in \mathcal{M}$ and $\xi \in D(\mathcal{M}, G, \alpha)$, we have that $\left(a^{\prime} \bar{\otimes} 1\right) D(\mathcal{M}, G, \alpha) \subset D(M, G, \alpha)$ and $\left(a^{\prime} \bar{\otimes} 1\right) \pi_{\alpha}(x) \xi=\pi_{\alpha}(x)\left(a^{\prime} \bar{\otimes} 1\right) \xi$.

$(2)\left(w_{t} \bar{\otimes} \rho_{t}\right) D(\mathcal{M}, G, \alpha) \subset D(\mathcal{M}, G, \alpha)$ and $\left(w_{t} \bar{\otimes} \rho_{t}\right) \pi_{\alpha}(x) \xi=\pi_{\alpha}(x)\left(w_{t} \bar{\otimes} \rho_{t}\right) \xi$, whenever $t \in G, x \in \mathcal{M}$ and $\xi \in D(\mathcal{M}, G, \alpha)$.

Proof. (1) This follows from the equalities

$$
\begin{gathered}
\left(\left(a^{\prime} \bar{\otimes} 1\right) \xi\right)(s)=a^{\prime} \xi(s), \text { and } \\
\alpha_{s^{-1}}(x)\left(\left(a^{\prime} \bar{\otimes} 1\right) \xi\right)(s)=a^{\prime} \alpha_{s^{-1}}(x) \xi(s)=\left(a^{\prime} \bar{\otimes} 1\right)\left(\pi_{\alpha}(x) \xi\right)(s), \quad s \in G,
\end{gathered}
$$

for all $a^{\prime} \in \mathcal{M}_{w}^{\prime}, \xi \in D_{0}(\mathcal{M}, G, \alpha)$ and $x \in \mathcal{M}$.

(2) We show that $\left(w_{t} \bar{\otimes} \rho_{t}\right) D_{0}(\mathcal{M}, G, \alpha) \subset D_{0}(\mathcal{M}, G, \alpha)$, for all $t \in G$. Indeed, this follows from the equalities

$$
\begin{gathered}
\left(\left(w_{t} \bar{\otimes} \rho_{t}\right) \xi\right)(s)=\Delta(t)^{\frac{1}{2}} w_{t} \xi(s t) \text { and } \\
\left(\alpha_{s^{-1}}(x)\left(w_{t} \bar{\otimes} \rho_{t}\right) \xi\right)(s)=\Delta(t)^{\frac{1}{2}} w_{t} \alpha_{(s t)^{-1}}(x) \xi(s t), \quad s \in G,
\end{gathered}
$$

for all $\xi \in D_{0}(\mathcal{M}, G, \alpha)$ and $x \in \mathcal{M}$. Furthermore, for any $t \in G, x \in \mathcal{M}$ and $\xi, \eta \in D_{0}(\mathcal{M}, G, \alpha)$, we have that

$$
\begin{aligned}
\left(\pi_{\alpha}(x)\left(w_{t} \bar{\otimes} \rho_{t}\right) \xi \mid \eta\right) & =\int_{G}\left(\alpha_{s^{-1}}(x)\left(w_{t} \bar{\otimes} \rho_{t}\right) \xi(s) \mid \eta(s)\right) d s \\
& =\int_{G} \Delta(t)^{\frac{1}{2}}\left(w_{t} \alpha_{(s t)^{-1}}(x) \xi(s t) \mid \eta(s)\right) d s \\
& =\int_{G}\left(\alpha_{s^{-1}}(x) \xi(s) \mid w_{t}^{*} \Delta(t)^{-\frac{1}{2}} \eta\left(s t^{-1}\right)\right) d s \\
& =\int_{G}\left(\alpha_{s^{-1}}(x) \xi(s) \mid w_{t}^{*}\left(\rho\left(t^{-1}\right) \eta\right)(s)\right) d s \\
& =\left(\pi_{\alpha}(x) \xi \mid\left(w_{t} \bar{\otimes} \rho_{t}\right)^{*} \eta\right),
\end{aligned}
$$

which implies that $\pi_{\alpha}(x)\left(w_{t} \bar{\otimes} \rho_{t}\right) \xi=\left(w_{t} \bar{\otimes} \rho_{t}\right) \pi_{\alpha}(x) \xi$. This completes the proof.

Definition 3.4. The $G W^{*}$-crossed product $\mathcal{M} \underset{\alpha}{\underset{\alpha}{x}} G$ of $\mathcal{M}$ by the action $\alpha$ of $G$ is defined as

$$
\mathcal{M} \underset{\alpha}{G W^{*}} G:=\left\{X \in \mathcal{L}^{\dagger}(D(\mathcal{M}, G, \alpha)) ; \bar{X} \eta\left(\mathcal{M}_{w}^{\prime}\right)^{\prime} \underset{\alpha}{\rtimes} G\right\} .
$$

Then we have the following

Theorem 3.5. $\mathcal{M} \underset{\alpha}{G W^{*}} G$ is a $G W^{*}$-algebra on $D(\mathcal{M}, G, \alpha)$ over $\left(\mathcal{M}_{w}^{\prime}\right)^{\prime}{ }_{\alpha} G$ containing $\mathcal{M} \stackrel{O^{*}}{\rtimes} G$. Moreover, if $\mathcal{M}$ is a regular $G W^{*}$-algebra, then $\mathcal{M} \stackrel{G W^{*}}{\rtimes} G$ is a regular $G W^{*}$-algebra over $\left(\mathcal{M}_{w}^{\prime}\right)^{\prime}{ }_{\alpha} G$.

Proof. By Lemmas 2.8, 3.1 and 3.3 we have

$$
\left(\left(\mathcal{M}_{w}^{\prime}\right)^{\prime}{ }_{\alpha} G\right)^{\prime} D(\mathcal{M}, G, \alpha) \subset D(\mathcal{M}, G, \alpha),
$$


which implies that $\mathcal{M} \underset{\alpha}{\underset{\sim}{\rtimes}} G$ is an $O^{*}$-algebra on $D(\mathcal{M}, G, \alpha)$. Furthermore, since $a^{\prime} \bar{\otimes} 1$ and $w_{t} \bar{\otimes} \rho_{t}$ commute with $\lambda(s)$, for each $a^{\prime} \in \mathcal{M}_{w}^{\prime}$ and $s, t \in G$, it follows from Lemma 3.3 that $\mathcal{M} \underset{\alpha}{O_{\alpha}^{*}} G \subset \mathcal{M} \underset{\alpha}{\stackrel{G W^{*}}{\rtimes}} G$, which implies that $\mathcal{M} \underset{\alpha}{G W^{*}} G$

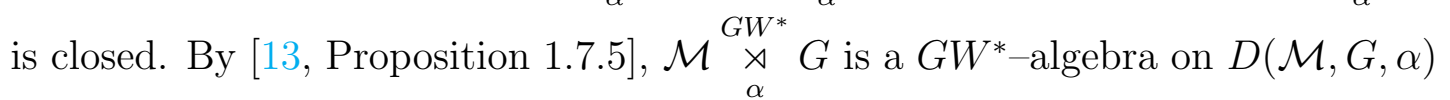
over $\left(\mathcal{M}_{w}^{\prime}\right)^{\prime} \underset{\alpha}{\rtimes} G$. Suppose now that $\mathcal{M}$ is a regular $G W^{*}$-algebra. It is clear that

$$
\left(\left(\mathcal{M}_{w}^{\prime}\right)^{\prime} \underset{\alpha}{\rtimes} G\right)^{\prime} \subset(\mathcal{M} \underset{\alpha}{\underset{\rtimes}{G}} G)_{w}^{\prime} \subset(\mathcal{M} \underset{\alpha}{\rtimes} G)_{w}^{O^{*}} \subset\left(\mathcal{M}_{b} \underset{\alpha}{\underset{\alpha}{O^{*}}} G\right)_{w}^{\prime} .
$$

Conversely, we show $\left(\mathcal{M}_{b} \underset{\alpha}{O^{*}} G\right)_{w}^{\prime} \subset\left(\left(\mathcal{M}_{w}^{\prime}\right)^{\prime} \underset{\alpha}{\rtimes} G\right)^{\prime}$. Indeed, it suffices to prove that $\pi_{\alpha}(a) C=C \pi_{\alpha}(a)$, for all $a \in\left(\mathcal{M}_{w}^{\prime}\right)^{\prime}$ and $C \in\left(\mathcal{M}_{b} \underset{\alpha}{\stackrel{O^{*}}{x}} G\right)_{w}^{\prime}$. Take an arbitrary $a \in\left(\mathcal{M}_{w}^{\prime}\right)^{\prime}$. Since, $\left(\overline{\mathcal{M}_{b}}\right)^{\prime \prime}=\left(\mathcal{M}_{w}^{\prime}\right)^{\prime}$, it follows from the Kaplansky density theorem that there exists a net $\left\{a_{i}\right\}$ in $\mathcal{M}_{b}$ such that $\left\|\bar{a}_{i}\right\| \leqq\|a\|$, for all $i$ and $\bar{a}_{i} \rightarrow a$ in the strong* topology. Hence, we have

$$
\left\|\alpha_{s^{-1}}\left(\bar{a}_{i}\right) \xi_{0}-\alpha_{s^{-1}}(a) \xi_{0}\right\| \leqq 2\|a\|\left\|\xi_{0}\right\|, \forall i, \forall s \in G \text { and } \forall \xi_{0} \in \mathcal{D},
$$

so using the Lebesgue convergence theorem, it is shown that $\lim _{i} \pi_{\alpha}\left(\bar{a}_{i}\right)\left(\xi_{0} \otimes f\right)=$ $\pi_{\alpha}(a)\left(\xi_{0} \otimes f\right)$, for each $\xi_{0} \in \mathcal{D}$ and $f \in \mathcal{C}_{c}(G)$. This implies that

$$
\begin{aligned}
\left(C \pi_{\alpha}(a)\left(\xi_{0} \otimes f\right) \mid \eta_{0} \otimes g\right) & =\lim _{i}\left(C \pi_{\alpha}\left(\bar{a}_{i}\right)\left(\xi_{0} \otimes f\right) \mid \eta_{0} \otimes g\right) \\
& =\lim _{i}\left(C\left(\xi_{0} \otimes f\right) \mid \pi_{\alpha}\left(a_{i}^{*}\right)\left(\eta_{0} \otimes g\right)\right) \\
& =\left(C\left(\xi_{0} \otimes f\right) \mid \pi_{\alpha}\left(a^{*}\right)\left(\eta_{0} \otimes g\right)\right),
\end{aligned}
$$

for all $C \in\left(\mathcal{M}_{b} \underset{\alpha}{\stackrel{O^{*}}{\alpha}} G\right)_{w}^{\prime}, \xi_{0}, \eta_{0} \in \mathcal{D}$ and $f, g \in \mathcal{C}_{c}(G)$. Hence, $C \in\left(\left(\mathcal{M}_{w}^{\prime}\right)^{\prime}{ }_{\alpha} G\right)^{\prime}$. Thus,

$$
\left(\left(\mathcal{M}_{w}^{\prime}\right)^{\prime} \underset{\alpha}{\rtimes} G\right)^{\prime}=\left(\mathcal{M} \underset{\alpha}{G W^{*}} G\right)_{w}^{\prime}=(\mathcal{M} \underset{\alpha}{\rtimes} G)_{w}^{O^{*}}=\left(\mathcal{M}_{b} \underset{\alpha}{\underset{\alpha}{O^{*}}} G\right)_{w}^{\prime},
$$

which implies that $\mathcal{M} \underset{\alpha}{\underset{\alpha}{\hookrightarrow W^{*}}} G$ is regular. This completes the proof.

At the end of this section we shall show that the crossed product $\mathcal{M} \underset{\alpha}{G W^{*}} G$ is the fixed point algebra in a $G W^{*}$-algebra defined by the tensor product $\left(\mathcal{M}_{w}^{\prime}\right)^{\prime} W^{W^{*}}$ $\mathcal{B}\left(L^{2}(G)\right)$ of the von Neumann algebras $\left(\mathcal{M}_{w}^{\prime}\right)^{\prime}$ and $\mathcal{B}\left(L^{2}(G)\right)$, for a certain action of $G$ on it. For the case of von Neumann algebras it follows from [32, Theorem 3.11] that

$$
\left(\mathcal{M}_{w}^{\prime}\right)^{\prime} \underset{\alpha}{\rtimes} G=\left\{A \in\left(\mathcal{M}_{w}^{\prime}\right)^{\prime} \stackrel{W}{*}^{*} \mathcal{B}\left(L^{2}(G)\right) ; \quad \theta_{t}(A)=A, \forall t \in G\right\},
$$

where $\theta$ is an action of $G$ on $\left(\mathcal{M}_{w}^{\prime}\right)^{\prime} \stackrel{W^{*}}{\otimes} \mathcal{B}\left(L^{2}(G)\right)$ defined by

$$
\theta_{t}(A)=\left(w_{t} \bar{\otimes} \rho_{t}\right) A\left(w_{t} \bar{\otimes} \rho_{t}\right)^{*}, t \in G .
$$


We generalize this to the case of $G W^{*}$-algebras. Since,

$$
\left(\left(\mathcal{M}_{w}^{\prime}\right)^{\prime}{ }^{W^{*}} \otimes \mathcal{B}\left(L^{2}(G)\right)\right)^{\prime} D(\mathcal{M}, G, \alpha)=\left(\mathcal{M}_{w}^{\prime} \stackrel{W^{*}}{\otimes} 1\right) D(\mathcal{M}, G, \alpha) \subset D(\mathcal{M}, G, \alpha)
$$

it follows that

$$
\left(\mathcal{M}_{w}^{\prime}\right)^{\prime} \underset{D(\mathcal{M}, G, \alpha)}{\stackrel{G W^{*}}{\otimes}} \mathcal{B}\left(L^{2}(G)\right):=\left\{X \in \mathcal{L}^{\dagger}(D(\mathcal{M}, G, \alpha)) ; \bar{X} \eta\left(\mathcal{M}_{w}^{\prime}\right)^{\prime}{ }^{W^{*}} \otimes \mathcal{B}\left(L^{2}(G)\right)\right\}
$$

is an $O^{*}$-algebra on $D(\mathcal{M}, G, \alpha)$. By (3.2) we have

$$
\begin{aligned}
\mathcal{M}_{\alpha} \underset{\alpha}{G W^{*}} G & =\left\{X \in \mathcal{L}^{\dagger}(D(\mathcal{M}, G, \alpha)) ; \bar{X} \eta\left(\mathcal{M}_{w}^{\prime}\right)^{\prime} \underset{\alpha}{\rtimes} G\right\} \\
& \subset\left\{X \in \mathcal{L}^{\dagger}(D(\mathcal{M}, G, \alpha)) ; \bar{X} \eta\left(\mathcal{M}_{w}^{\prime}\right)^{\prime}{ }^{W^{*}} \otimes \mathcal{B}\left(L^{2}(G)\right)\right\} \\
& =\left(\mathcal{M}_{w}^{\prime}\right)^{\prime} \underset{D(\mathcal{M}, G, \alpha)}{\stackrel{G W^{*}}{\otimes}} \mathcal{B}\left(L^{2}(G)\right),
\end{aligned}
$$

which implies that $\left(\mathcal{M}_{w}^{\prime}\right)^{\prime} \underset{D(\mathcal{M}, G, \alpha)}{G W^{*}} \mathcal{B}\left(L^{2}(G)\right)$ is closed. Hence, $\left(\mathcal{M}_{w}^{\prime}\right)^{\prime} \underset{D(\mathcal{M}, G, \alpha)}{G W^{*}}$ $\mathcal{B}\left(L^{2}(G)\right)$ is a $G W^{*}$-algebra on $D(\mathcal{M}, G, \alpha)$ over $\left(\mathcal{M}_{w}^{\prime}\right)^{\prime} W^{*} \mathcal{B}\left(L^{2}(G)\right)$ and it is called the $G W^{*}$-tensor product defined by $\left(\mathcal{M}_{w}^{\prime}\right)^{\prime} W^{*} \otimes \mathcal{B}\left(L^{2}(G)\right)$ and $D(\mathcal{M}, G, \alpha)$. For more details concerning $G W^{*}$-tensor products, the reader is referred to [6].

We extend now the action $\theta$ of $G$ on $\left(\mathcal{M}_{w}^{\prime}\right)^{\prime} W^{*} \otimes \mathcal{B}\left(L^{2}(G)\right)$ to the action of $G$ on the $G W^{*}$-tensor product $\left(\mathcal{M}_{w}^{\prime}\right)^{\prime} \underset{D(\mathcal{M}, G, \alpha)}{\stackrel{G W^{*}}{\otimes}} \mathcal{B}\left(L^{2}(G)\right)$ by

$$
\theta_{t}(X)=\left(w_{t} \bar{\otimes} \rho_{t}\right) X\left(w_{t} \bar{\otimes} \rho_{t}\right)^{*}, t \in G, X \in\left(\mathcal{M}_{w}^{\prime}\right)^{\prime} \underset{D(\mathcal{M}, G, \alpha)}{\stackrel{G W^{*}}{\otimes}} \mathcal{B}\left(L^{2}(G)\right) .
$$

Proposition 3.6. The following holds true:

$$
\mathcal{M} \underset{\alpha}{G W^{*}} G=\left\{X \in\left(\mathcal{M}_{w}^{\prime}\right)^{\prime} \underset{D(\mathcal{M}, G, \alpha)}{\stackrel{G W^{*}}{\otimes}} \mathcal{B}\left(L^{2}(G)\right) ; \theta_{t}(X)=X, \forall t \in G\right\} .
$$

Proof. Take an arbitrary $X \in \mathcal{M} \underset{\alpha}{\stackrel{G W^{*}}{\rtimes}} G$. By (3.3), $X \in\left(\mathcal{M}_{w}^{\prime}\right)^{\prime} \underset{D(\mathcal{M}, G, \alpha)}{G W^{*}} \mathcal{B}\left(L^{2}(G)\right)$ and by (3.2) there exists a sequence $\left\{X_{n}\right\}$ in $\left(\mathcal{M}_{w}^{\prime}\right)^{\prime} W^{*} \otimes \mathcal{B}\left(L^{2}(G)\right)$ such that $\theta_{t}\left(X_{n}\right)=X_{n}$, for all $t \in G$ and $X_{n} \rightarrow X$ strongly on $D(\mathcal{M}, G, \alpha)$. Since, $\theta_{t}\left(X_{n}\right) \rightarrow \theta_{t}(X)$ strongly on $D(\mathcal{M}, G, \alpha)$, for all $t \in G$, it follows that $\theta_{t}(X)=X$, for all $t \in G$.

Conversely, take an arbitrary $X \in\left(\mathcal{M}_{w}^{\prime}\right)^{\prime} \underset{D(\mathcal{M}, G, \alpha)}{\stackrel{G W^{*}}{\otimes}} \mathcal{B}\left(L^{2}(G)\right)$, such that $\theta_{t}(X)=$ $X$, for all $t \in G$. Let $\bar{X}=U|\bar{X}|$ be the polar decomposition of $\bar{X}$ and $|\bar{X}|=$ $\int_{0}^{\infty} \lambda d E(\lambda)$ the spectral resolution of $|\bar{X}|$. Put $X_{n}=\bar{X} E(n), n \in \mathbb{N}$. Then $X_{n} \in$ $\left(\mathcal{M}_{w}^{\prime}\right)^{\prime} W^{*} \otimes \mathcal{B}\left(L^{2}(G)\right)$, for each $n \in \mathbb{N}$. Let $t \in G$. Since, $\bar{X}=\theta_{t}(\bar{X})=\theta_{t}(U) \theta_{t}(|\bar{X}|)$, it follows by the uniqueness of the polar decomposition of $\bar{X}$ that $\theta_{t}(U)=U$ and $\theta_{t}(|\bar{X}|)=|\bar{X}|$. Furthermore, since $|\bar{X}|=\theta_{t}(|\bar{X}|)=\int_{0}^{\infty} \lambda d \theta_{t}(E(\lambda))$, by the uniqueness of the spectral resolution of $|X|$ we have that $\theta_{t}(E(\lambda))=E(\lambda)$, for all 
$\lambda \geq 0$. Therefore, $\theta_{t}\left(X_{n}\right)=X_{n}$, for all $n \in \mathbb{N}$ and $t \in G$, which by (3.2) implies that $X_{n} \in\left(\mathcal{M}_{w}^{\prime}\right)^{\prime} \rtimes G$, for each $n \in \mathbb{N}$. Since, $X_{n} \rightarrow X$ strongly on $D(\mathcal{M}, G, \alpha)$, we have $\bar{X} \eta\left(\mathcal{M}_{w}^{\prime}\right)^{\prime} \underset{\alpha}{\rtimes} G$. Hence, $X \in \mathcal{M}_{\underset{\alpha}{\rtimes}}^{G W^{*}} G$ by (3.1). This completes the proof.

\section{Duality}

Throughout this section, $\mathcal{M}$ is a closed $O^{*}$-algebra on $\mathcal{D}$ in $\mathcal{H}$ such that $\mathcal{M}_{w}^{\prime} \mathcal{D} \subset \mathcal{D}, G$ is an abelian locally compact group with Haar measure $d s$ and $\alpha$ a spatial action of $G$ on $\mathcal{M}$, that is, $\alpha_{s}(x)=w_{s} x w_{s}^{*}$, where $w$ is a strongly continuous unitary representation of $G$ on $\mathcal{H}$ such that $w_{s} \mathcal{D} \subset \mathcal{D}$, for each $s \in G$. Let $\widehat{G}$ be the dual group of $G$, that is, the locally compact group of all characters of $G$ and let $d p$ denote the Haar measure of $\widehat{G}$. Given $p \in \widehat{G}, s \in G$, and $f \in L^{2}(G)$ we set

$$
\left(m_{p} f\right)(s)=\overline{<s, p>} f(s),
$$

where $\left\langle s, p>\right.$ is the value of $p$ at $s$. Then $m: p \longmapsto m_{p}$ is a strongly continuous unitary representation of $\widehat{G}$ on $L^{2}(G)$, whose properties are listing in the following

Lemma 4.1. (1) $m_{p} \lambda_{t} m_{p}^{*}=\overline{<t, p>} \lambda_{t}$, for all $t \in G$ and $p \in \widehat{G}$.

(2) $\left(\mathbb{I} \bar{\otimes} m_{p}\right) D_{0}(\mathcal{M}, G, \alpha)=D_{0}(\mathcal{M}, G, \alpha),\left(I \bar{\otimes} m_{p}\right) D(\mathcal{M}, G, \alpha)=D(\mathcal{M}, G, \alpha)$, and $\left(\mathbb{I} \bar{\otimes} m_{p}\right) \pi_{\alpha}(x)\left(\mathbb{I} \bar{\otimes} m_{p}\right)^{*} \xi=\pi_{\alpha}(x) \xi$, for all $\xi \in D(\mathcal{M}, G, \alpha), x \in \mathcal{M}$, and $p \in \widehat{G}$.

(3) $\left(w_{t} \bar{\otimes} \rho_{t}\right)\left(\mathbb{I} \bar{\otimes} m_{p}\right)\left(w_{t} \bar{\otimes} \rho_{t}\right)^{*}=\overline{<t, p>}\left(\mathbb{I} \bar{\otimes} m_{p}\right)$, for all $t \in G$ and $p \in \widehat{G}$.

Proof. (1) This follows by a short direct calculation.

(2) Since,

$$
\left(\left(1 \bar{\otimes} m_{p}\right)\left(\xi_{0} \otimes g\right)\right)(s)=\overline{<s, p>} g(s) \xi_{0}=\overline{<s, p>}\left(\xi_{0} \otimes g\right)(s),
$$

for all $s \in G, p \in \widehat{G}, \xi_{0} \in \mathcal{D}$, and $g \in \mathcal{C}_{c}(G)$, it follows that

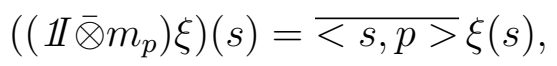

for all $\xi \in L^{2}(G, \mathcal{H})$. Clearly $\left(\mathbb{I} \bar{\otimes} m_{p}\right)^{*}=\mathbb{I} \bar{\otimes} m_{p^{-1}}$. Hence,

$$
\begin{aligned}
\left(\mathbb{I} \bar{\otimes} m_{p}\right) D_{0}(\mathcal{M}, G, \alpha) & =D_{0}(\mathcal{M}, G, \alpha) \text { and } \\
\left(\left(\mathbb{I} \bar{\otimes} m_{p}\right) \pi_{\alpha}(x) \xi\right)(s) & =\overline{<s, p>} \alpha_{s^{-1}}(x) \xi(s) \\
& =\alpha_{s^{-1}}(x)\left(\left(\mathbb{I} \bar{\otimes} m_{p}\right) \xi\right)(s)=\left(\pi_{\alpha}(x)\left(\mathbb{I} \bar{\otimes} m_{p}\right) \xi\right)(s),
\end{aligned}
$$

for all $s \in G, x \in \mathcal{M}$, and $\xi \in D_{0}(\mathcal{M}, G, \alpha)$. To complete the proof of assertion (2), we show that the last equation is also satisfied for $\xi \in D(\mathcal{M}, G, \alpha)$. For this we consider a net $\left\{\xi_{i}\right\}$ in $D_{0}(\mathcal{M}, G, \alpha)$ which converges to $\xi$ under the graph topology generated by $\mathcal{M} \underset{\alpha}{\underset{\sim}{O^{*}}} G$. Then by using Lemma 2.8 several times, we 
obtain

$$
\begin{gathered}
\left(\mathbb{I} \bar{\otimes} m_{p}\right) \pi_{\alpha}(x) \xi=\lim _{i}\left(\mathbb{I} \bar{\otimes} m_{p}\right) \pi_{\alpha}(x) \xi_{i}=\lim _{i} \pi_{\alpha}(x)\left(I \bar{\otimes} m_{p}\right) \xi_{i}, \\
\left(\mathbb{I} \bar{\otimes} m_{p}\right) \pi_{\alpha}(x) \xi=\overline{\pi_{\alpha}(x)}\left(\mathbb{I} \bar{\otimes} m_{p}\right) \xi, \\
\left(\mathbb{I} \bar{\otimes} m_{p}\right) \xi \in D(\mathcal{M}, G, \alpha) \text { and }\left(\mathbb{I} \bar{\otimes} m_{p}\right) \pi_{\alpha}(x) \xi=\pi_{\alpha}(x)\left(\mathbb{I} \bar{\otimes} m_{p}\right) \xi,
\end{gathered}
$$

which proves assertion (2).

(3) Given $t \in G, p \in \widehat{G}, g \in \mathcal{C}_{c}(G)$, and $\xi_{0} \in \mathcal{H}$, we have

$$
\begin{aligned}
& \left(\left(\mathbb{I} \bar{\otimes} m_{p}\right)\left(w_{t} \bar{\otimes} \rho_{t}\right)^{*}\left(\xi_{0} \otimes g\right)\right)(s)=\left(\left(\mathbb{I} \bar{\otimes} m_{p}\right)\left(w_{t^{-1}} \bar{\otimes} \rho_{t^{-1}}\right)\left(\xi_{0} \otimes g\right)\right)(s) \\
& =\overline{<s, p>} g\left(s t^{-1}\right) w_{t^{-1}} \xi_{0}=\overline{<t, p>} \overline{<s t^{-1}, p>} g\left(s t^{-1}\right) w_{t^{-1}} \xi_{0} \\
& =\overline{<t, p>}\left(w_{t} \bar{\otimes} \rho_{t}\right)^{*}\left(1 \bar{\otimes} m_{p}\right)\left(\xi_{0} \otimes g\right)(s) \text {. }
\end{aligned}
$$

Since, the linear span of elements of the form $\xi_{0} \otimes g$ is dense in $L^{2}(G, \mathcal{H})$, this implies assertion (3).

Definition 4.2. The dual action $\widehat{\alpha}$ of the dual group $\widehat{G}$ on $\mathcal{L}^{\dagger}(D(\mathcal{M}, G, \alpha))$ is defined by $\widehat{\alpha_{p}}(X)=\left(\mathbb{I} \bar{\otimes} m_{p}\right) X\left(\mathbb{I} \bar{\otimes} m_{p}\right)^{*}, X \in \mathcal{L}^{\dagger}(D(\mathcal{M}, G, \alpha))$.

Lemma 4.3. For each $p \in \widehat{G}, \widehat{\alpha_{p}}\left(\mathcal{M} \underset{\alpha}{O_{\alpha}^{*}} G\right) \subset \mathcal{M} \underset{\alpha}{O_{\alpha}^{*}} G$, and $\widehat{\alpha_{p}}\left(\mathcal{M} \underset{\alpha}{G W^{*}} G\right) \subset$ $\mathcal{M} \stackrel{\substack{\rtimes \\ \alpha}}{W^{*}} G$, so $\widehat{\alpha}$ is a spatial action of $\widehat{G}$ on $\mathcal{M} \underset{\alpha}{\underset{\alpha}{O^{*}}} G$ and on $\mathcal{M} \underset{\alpha}{G W^{*}} G$. Moreover, the action $\widehat{\alpha}$ of $\widehat{G}$ on $\mathcal{M}^{O^{*}} G$ is strongly continuous.

Proof. As a consequence of Lemma 4.1, $\widehat{\alpha_{p}}(X) \in \mathcal{M} \underset{\alpha}{O_{\alpha}^{*}} G$, whenever $X \in \mathcal{M} \underset{\alpha}{O^{*}} G$. To show that $\widehat{\alpha_{p}}\left(\mathcal{M} \underset{\alpha}{G W^{*}} G\right) \subset \mathcal{M} \underset{\alpha}{G W^{*}} G$, let $X \in \mathcal{M} \underset{\alpha}{G W^{*}} G$ be fixed. By Proposition 3.6, $X \in \mathcal{L}^{\dagger}(D(\mathcal{M}, G, \alpha)), \bar{X} \eta\left(\mathcal{M}_{w}^{\prime}\right)^{\prime} \stackrel{W}{*}^{*} \mathcal{B}\left(L^{2}(G)\right)$ and $\theta_{t}(X)=X$, for all $t \in G$. We show that $\widehat{\alpha_{p}}(X)=\left(\mathbb{I} \bar{\otimes} m_{p}\right) X\left(I \bar{\otimes} m_{p}\right)^{*}$ has the same properties. Indeed, it belongs to $\mathcal{L}^{\dagger}(D(\mathcal{M}, G, \alpha))$ by Lemma $4.1(2) ; \overline{\alpha_{p}}(X)=\left(I \bar{\otimes} m_{p}\right) \bar{X}\left(\mathbb{I} \bar{\otimes} m_{p}\right)^{*}$ is affiliated to $\left(\mathcal{M}_{w}^{\prime}\right)^{\prime} \stackrel{W}{*}^{\otimes} \mathcal{B}\left(L^{2}(G)\right)$, since, $\left(\mathbb{I} \bar{\otimes} m_{p}\right) \in\left(\mathcal{M}_{w}^{\prime}\right)^{\prime} \stackrel{W}{*}^{*} \mathcal{B}\left(L^{2}(G)\right)$. Since, Lemma 4.1 (3) implies that $\left(w_{t} \bar{\otimes} \rho_{t}\right)\left(\Pi \bar{\otimes} m_{p}\right)=\overline{\langle t, p>}\left(\mathbb{I} \bar{\otimes} m_{p}\right)\left(w_{t} \bar{\otimes} \rho_{t}\right)$ and $\left(I \bar{\otimes} m_{p}\right)^{*}\left(w_{t} \bar{\otimes} \rho_{t}\right)^{*}=<t, p>\left(w_{t} \bar{\otimes} \rho_{t}\right)^{*}\left(I \bar{\otimes} m_{p}\right)^{*}$, we also obtain

$$
\begin{aligned}
& \theta_{t}\left(\widehat{\alpha_{p}}(X)\right)=\left(w_{t} \bar{\otimes} \rho_{t}\right)\left(\mathbb{I} \bar{\otimes} m_{p}\right) X\left(\mathbb{I} \bar{\otimes} m_{p}\right)^{*}\left(w_{t} \bar{\otimes} \rho_{t}\right)^{*} \\
& =\overline{<t, p>}<t, p>\left(\mathbb{I} \bar{\otimes} m_{p}\right)\left(w_{t} \bar{\otimes} \rho_{t}\right) X\left(w_{t} \bar{\otimes} \rho_{t}\right)^{*}\left(\mathbb{I} \bar{\otimes} m_{p}\right)^{*} \\
& =\widehat{\alpha_{p}}\left(\theta_{t}(X)\right)=\widehat{\alpha_{p}}(X) \text {. }
\end{aligned}
$$

By applying again Proposition 3.6, we see that $\widehat{\alpha_{p}}(X) \in \mathcal{M} \stackrel{G W^{*}}{\rtimes} G$.

To show that $\widehat{\alpha}$ is strongly continuous on $\mathcal{M}_{\rtimes}^{O^{*}} G$, we use the fact, that $\mathcal{M}_{\rtimes}^{O^{*}} G$ is the linear span of elements of the form $\pi_{\alpha}(x) \lambda(t), x \in \mathcal{M}, t \in G$. For those 
elements Lemma 4.1 implies that $\widehat{\alpha_{p}}\left(\pi_{\alpha}(x) \lambda(t)\right)=\overline{\langle t, p>} \pi_{\alpha}(x) \lambda(t)$. Hence,

$$
\begin{aligned}
& \lim _{p \rightarrow p_{0}}\left\|\left(\widehat{\alpha_{p}}\left(\pi_{\alpha}(x) \lambda(t)\right) \xi-\widehat{\alpha_{p_{0}}}\left(\pi_{\alpha}(x) \lambda(t)\right) \xi\right)\right\|= \\
& \lim _{p \rightarrow p_{0}} \mid \overline{\langle t, p>}-\overline{<t, p_{0}>\mid} \cdot\left\|\left(\pi_{\alpha}(x) \lambda(t)\right) \xi\right\|=0,
\end{aligned}
$$

for all $x \in \mathcal{M}, t \in G, p_{0} \in \widehat{G}$, and $\xi \in D(\mathcal{M}, G, \alpha)$. This implies that the action $\widehat{\alpha}$ is strongly continuous on $\mathcal{M}_{\alpha}^{\rtimes} G$.

We may now consider the crossed products

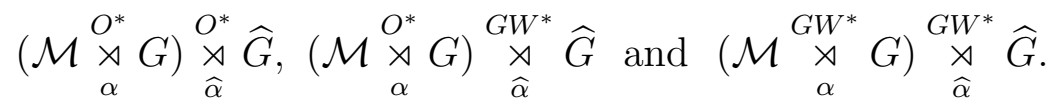

Note that Lemma 2.6 implies that $\lambda(t) \pi_{\alpha}(x)=\pi_{\alpha}\left(\alpha_{t}(x)\right) \lambda(t)$, for all $x \in \mathcal{M}$ and $t \in G$. Using also (1) and (2) of Lemma 4.1, we conclude that for each $L^{2}(G, \mathcal{H})$-valued function $\zeta$ on $\widehat{G}$ the function

$$
\left(\widehat{\alpha_{p^{-1}}}\left(\pi_{\alpha}(x) \lambda(t)\right) \zeta\right)(p)=\overline{<t, p^{-1}>} \pi_{\alpha}(x) \lambda(t) \zeta(p)=<t, p>\lambda(t) \pi_{\alpha}\left(\alpha_{t^{-1}}(x)\right) \zeta(p)
$$

depends continuously on $p \in \widehat{G}$, if and only if $p \rightarrow \pi_{\alpha}\left(\alpha_{t^{-1}}(x)\right) \zeta(p)$ is continuous. Hence,

$$
\begin{aligned}
& D_{0}\left(\mathcal{M} \underset{\alpha}{O^{*}} G, \widehat{G}, \widehat{\alpha}\right)=\left\{\zeta \in \mathcal{C}_{c}\left(\widehat{G}, L^{2}(G, \mathcal{H})\right) ; \zeta(p) \in D(\mathcal{M}, G, \alpha),\right. \\
& \quad \text { for all } p \in \widehat{G} \text { and the } \operatorname{map} \widehat{G} \ni p \rightarrow \pi_{\alpha}(x) \zeta(p) \in L^{2}(G, \mathcal{H}) \\
& \quad \text { is continuous, for all } x \in \mathcal{M}\} .
\end{aligned}
$$

Since, $\widehat{\alpha}$ is strongly continuous on $\mathcal{M}^{O^{*}} G$ by Lemma 4.3, it follows from Proposition 2.9 that

$$
\begin{aligned}
& D_{0}(\mathcal{M}, G, \alpha) \otimes \mathcal{C}_{c}(\widehat{G}) \text { is dense in } D\left(\mathcal{M} \underset{\alpha}{O^{*}} G, \widehat{G}, \widehat{\alpha}\right)= \\
& D\left(\left(\mathcal{M} \underset{\alpha}{O_{\alpha}^{*}} G\right) \underset{\widehat{\alpha}}{\underset{O^{*}}{\rtimes}} \widehat{G}\right)=D\left(\left(\mathcal{M} \underset{\alpha}{O_{\alpha}^{*}} G\right) \underset{\widehat{\alpha}}{\stackrel{G W^{*}}{\rtimes}} \widehat{G}\right) \text { w.r.t. }
\end{aligned}
$$

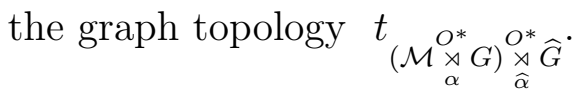

Clearly, $\left.\left(\mathcal{M} \underset{\alpha}{\stackrel{O^{*}}{\rtimes}} G\right) \underset{\widehat{\alpha}}{\stackrel{O^{*}}{\rtimes}} \widehat{G}\right) \subset\left(\mathcal{M} \underset{\alpha}{\left.\underset{\rtimes}{O^{*}} G\right)} \underset{\widehat{\alpha}}{\stackrel{G W^{*}}{\rtimes}} \widehat{G}\right.$.

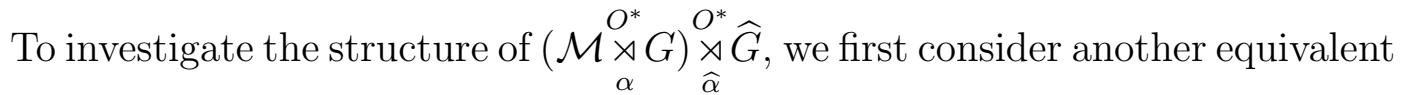
construction of it.

Lemma 4.4. For $x \in \mathcal{M}, \xi \in D_{0}(\mathcal{M}, G, \alpha)$, and $s \in G$, we put

$$
\left[L_{x}^{0} W \xi\right](s)=x(W \xi)(s) .
$$

Then $L_{x}^{0}$ is a linear operator on $W D_{0}(\mathcal{M}, G, \alpha)$ and $L_{x}^{0} W \xi=W \pi_{\alpha}(x) \xi$, for all $x \in \mathcal{M}$ and $\xi \in D_{0}(\mathcal{M}, G, \alpha) . L^{0}: x \longmapsto L_{x}^{0}$ is a*-representation of $\mathcal{M}$ on $W D_{0}(\mathcal{M}, G, \alpha)$ and $L_{\mathcal{M}}^{0}:=\left\{L_{x}^{0}: x \in \mathcal{M}\right\}$ is an $O^{*}$-algebra on $W D_{0}(\mathcal{M}, G, \alpha)$. 
Proof. Take arbitrary $x \in \mathcal{M}$ and $\xi \in D_{0}(\mathcal{M}, G, \alpha)$. Since, $(W \xi)(s)=w_{s} \xi(s) \in$ $\mathcal{D}$ and $\left(L_{x}^{0} W \xi\right)(s)=x w_{s} \xi(s)=w_{s} \pi_{\alpha}(x) \xi(s)$, for all $s \in G$, it follows that $L_{x}^{0} W \xi=$ $W \pi_{\alpha}(x) \xi$ and $L_{x}^{0} W D_{0}(\mathcal{M}, G, \alpha) \subset W D_{0}(\mathcal{M}, G, \alpha)$. Then it is easy to see that $L^{0}$ is a $*$-representation of $\mathcal{M}$ on $W D_{0}(\mathcal{M}, G, \alpha)$.

Lemma 4.5. For each $x \in \mathcal{M}, \xi \in D_{0}(\mathcal{M}, G, \alpha)$ and $t \in G$ we have

$$
\pi_{\alpha}(x) \xi=W^{*} L_{x}^{0} W \xi \text { and } \lambda(t) \xi=W^{*}\left(w_{t} \bar{\otimes} \lambda_{t}\right) W \xi .
$$

Hence, we also have

$$
\bigcap_{x \in \mathcal{M}} \mathcal{D}\left(\overline{L_{x}^{0}}\right)=W D(M, G, \alpha), L_{x}:=\overline{L_{x}^{0}} \uparrow_{W D(M, G, \alpha)}=W \pi_{\alpha}(x) W^{*} .
$$

In particular, the $O^{*}$-algebra $\mathfrak{M}$ generated by $\left\{\pi_{\alpha}^{0}(x) \lambda_{t} ; x \in \mathcal{M}, t \in G\right\}$ is spatially isomorphic to the $O^{*}$-algebra on $W D_{0}(\mathcal{M}, G, \alpha)$ generated by the operators

$$
\left\{L_{x}^{0}, w_{t} \bar{\otimes} \lambda_{t} \uparrow_{W D_{0}(\mathcal{M}, G, \alpha)} ; x \in \mathcal{M}, t \in G\right\}
$$

and its closure $\mathcal{M}_{\alpha}^{O_{\alpha}^{*}} G$ is spatially isomorphic to the $O^{*}$-algebra on $W D(M, G, \alpha)$ generated by the operators

$$
\left\{L_{x}, w_{t} \bar{\otimes} \lambda_{t} \uparrow_{W D(M, G, \alpha)} ; x \in \mathcal{M}, t \in G\right\} .
$$

Proof. By Lemma 4.4 we have that

$$
L_{x}^{0} W D_{0}(\mathcal{M}, G, \alpha) \subset W D_{0}(\mathcal{M}, G, \alpha) \text { and } W^{*} L_{x}^{0} W \xi=\pi_{\alpha}(x) \xi,
$$

for all $x \in \mathcal{M}$ and $\xi \in D_{0}(\mathcal{M}, G, \alpha)$, which implies that

$$
\bigcap_{x \in \mathcal{M}} \mathcal{D}\left(\overline{L_{x}^{0}}\right)=W D(M, G, \alpha) \text { and } L_{x}=W \pi_{\alpha}(x) W^{*} .
$$

Moreover, given $s, t \in G$ and $\xi \in D_{0}(\mathcal{M}, G, \alpha)$, we have

$$
\begin{aligned}
(\lambda(t) \xi)(s)=\xi\left(t^{-1} s\right)=w_{s^{-1} t} w_{t^{-1}} \xi\left(t^{-1} s\right) & =w_{s}^{*}\left(\left(w_{t} \bar{\otimes} \lambda_{t}\right) W \xi\right)(s) \\
& =\left(W^{*}\left(w_{t} \bar{\otimes} \lambda_{t}\right) W \xi\right)(s),
\end{aligned}
$$

which implies that $\left(w_{t} \bar{\otimes} \lambda_{t}\right) W \xi=W \lambda(t) \xi \in W D(M, G, \alpha)$. This completes the proof.

Note that the correspondence

$$
\bigoplus_{\iota \in J} \mathcal{H}_{\iota} \ni\left\{\xi_{\iota}^{0}\right\} \longrightarrow \sum_{\iota \in J} \xi_{\iota}^{0} \otimes \varphi_{\iota} \in \mathcal{H} \bar{\otimes} L^{2}(G) \cong L^{2}(G, \mathcal{H})
$$

where $\left\{\varphi_{\iota}\right\}_{\iota \in J}$ is a complete orthonormal system in $L^{2}(G)$ and $\mathcal{H}_{\iota}=\mathcal{H}$, for all $\iota \in J$, establishes a unitary equivalence of the direct sum $\bigoplus_{\iota \in J} \mathcal{H}$ of Hilbert spaces and the tensor product $\mathcal{H} \bar{\otimes} L^{2}(G)$.

Lemma 4.6. For each $x \in \mathcal{M}$, we have

$$
\begin{gathered}
\mathcal{D}\left(L_{x}^{*}\right)=\left\{\sum_{\iota \in J} \xi_{\iota}^{0} \otimes \varphi_{\iota} ; \xi_{\iota}^{0} \in D\left(x^{*}\right) \text { and } \sum_{\iota \in J}\left\|x^{*} \xi_{\iota}^{0}\right\|^{2}<\infty\right\} \text { and } \\
L_{x}^{*}\left(\sum_{\iota \in J} \xi_{\iota}^{0} \otimes \varphi_{\iota}\right)=\sum_{\iota \in J} x^{*} \xi_{\iota}^{0} \otimes \varphi_{\iota}, \text { whenever } \sum_{\iota \in J} \xi_{\iota}^{0} \otimes \varphi_{\iota} \in \mathcal{D}\left(L_{x}^{*}\right) .
\end{gathered}
$$


Moreover,

$$
L_{x}^{*}=(x \otimes 1)^{*}=\overline{x^{*} \otimes 1} \text { and } \overline{L_{x}}=\overline{x \otimes 1}=\left(x^{*} \otimes 1\right)^{*} .
$$

Proof. Since, $W^{*}\left(\mathcal{D} \otimes L^{2}(G)\right) \subset D(\mathcal{M}, G, \alpha), L_{x} W W^{*}\left(\xi_{0} \otimes f\right)=(x \otimes 1)\left(\xi_{0} \otimes f\right)$, for all $\xi_{0} \in \mathcal{D}$ and $f \in L^{2}(G)$, that is, $x \otimes 1 \subset L_{x}$. Consequently, $L_{x}^{*} \subset(x \otimes 1)^{*}$. Suppose that $\sum_{\iota \in J} \xi_{\iota}^{0} \otimes \varphi_{\iota} \in \mathcal{D}\left((x \otimes 1)^{*}\right)$ and that $(x \otimes 1)^{*}\left(\sum_{\iota \in J} \xi_{\iota}^{0} \otimes \varphi_{\iota}\right)=$ $\sum_{\iota \in J} \zeta_{\iota}^{0} \otimes \varphi_{\iota} \in \mathcal{H} \bar{\otimes} L^{2}(G) \cong L^{2}(G, \mathcal{H})$. Then

$$
\left((x \otimes 1)\left(\xi_{0} \otimes f\right) \mid \sum_{\iota \in J} \xi_{\iota}^{0} \otimes \varphi_{\iota}\right)=\left(\left(\xi_{0} \otimes f\right) \mid \sum_{\iota \in J} \zeta_{\iota}^{0} \otimes \varphi_{\iota}\right),
$$

for all $\xi_{0} \in \mathcal{D}$ and $f \in L^{2}(G)$. This implies

$$
\left(x \xi_{0} \mid \xi_{\iota}^{0}\right)=\left(\xi_{0} \mid \zeta_{\iota}^{0}\right) \text { and consequently } x^{*} \xi_{\iota}^{0}=\zeta_{\iota}^{0},
$$

for all $\iota \in J$. Hence,

$$
\sum_{\iota \in J}\left\|x^{*} \xi_{\iota}^{0}\right\|^{2}=\sum_{\iota \in J}\left\|\zeta_{\iota}^{0}\right\|^{2}<\infty \text { and }(x \otimes 1)^{*}\left(\sum_{\iota \in J} \xi_{\iota}^{0} \otimes \varphi_{\iota}\right)=\sum_{\iota \in J} x^{*} \xi_{\iota}^{0} \otimes \varphi_{\iota} .
$$

Conversely, given $\xi_{\iota}^{0} \in \mathcal{D}\left(x^{*}\right)$ with $\sum_{\iota \in J}\left\|x^{*} \xi_{\iota}^{0}\right\|^{2}<\infty$, it is easy to see that $\sum_{\iota \in J} \xi_{\iota}^{0} \otimes \varphi_{\iota} \in \mathcal{D}\left(L_{x}^{*}\right)$ and $L_{x}^{*}\left(\sum_{\iota \in J} \xi_{\iota}^{0} \otimes \varphi_{\iota}\right)=\sum_{\iota \in J} x^{*} \xi_{\iota}^{0} \otimes \varphi_{\iota}$, which completes the proof.

By Lemmas 4.5 and 4.6 we have

$$
\begin{gathered}
\bigcap_{x \in \mathcal{M}} \mathcal{D}(\overline{x \otimes 1})=\bigcap_{x \in \mathcal{M}} \mathcal{D}\left(\overline{L_{x}}\right)=W D(\mathcal{M}, G, \alpha) \text { and } \\
\overline{x \otimes 1} \Upsilon_{\bigcap_{y \in \mathcal{M}} \mathcal{D}(\overline{y \otimes 1})}=L_{x}, x \in \mathcal{M} ;
\end{gathered}
$$

so we may denote $L_{x}$ by $x \widetilde{\otimes} 1$. By Lemmas $4.4,4.5$, and 4.6 we have the following

Theorem 4.7. $\mathcal{M}_{\alpha}^{O^{*}} G$ is spatially isomorphic to the closed $O^{*}$-algebra on the domain $W D(\mathcal{M}, G, \alpha)$ generated by the operators $\left\{x \widetilde{\otimes} 1, w_{s} \bar{\otimes} \lambda_{s} ; x \in \mathcal{M}, s \in G\right\}$, and it is self-adjoint if and only if $\mathcal{M}$ is self-adjoint.

Proof. It is sufficient to show that $\mathcal{M}$ is self-adjoint if and only if $\mathcal{M} \stackrel{O^{*}}{\rtimes} G$ has this property. Suppose that $\mathcal{M}$ is self-adjoint. Take an arbitrary $\zeta=\sum_{\iota}^{\alpha} \zeta_{\iota}^{0} \varphi_{\iota} \in$ $\bigcap_{x \in \mathcal{M}} \mathcal{D}\left((x \otimes 1)^{*}\right)$. Then it follows from Lemma 4.6 that $\zeta_{\iota}^{0} \in \mathcal{D}\left(x^{*}\right)$, for all indices $\iota$ and $x \in \mathcal{M}$. By the self-adjointness of $\mathcal{M}$, this implies that $\zeta_{\iota}^{0} \in \mathcal{D}$, for all $\iota$ and $\sum_{\iota}\left\|x \zeta_{\iota}^{0}\right\|^{2}<\infty$, for all $x \in \mathcal{M}$. Hence, $\zeta \in \bigcap_{x \in \mathcal{M}} \mathcal{D}(\overline{x \otimes 1})$ and so $\bigcap_{x \in \mathcal{M}} \mathcal{D}\left((x \otimes 1)^{*}\right) \subset \bigcap_{x \in \mathcal{M}} \mathcal{D}(\overline{x \otimes 1})$. The converse inclusion is trivial. Hence, we have

$$
\bigcap_{x \in \mathcal{M}} \mathcal{D}\left((x \otimes 1)^{*}\right)=\bigcap_{x \in \mathcal{M}} \mathcal{D}(\overline{x \otimes 1})=W D(\mathcal{M}, G, \alpha)
$$


which implies that

$$
\begin{aligned}
\mathcal{D}^{*}\left(\mathcal{M}_{\alpha}^{\rtimes} \underset{O^{*}}{\rtimes} G\right)=\bigcap_{x \in \mathcal{M}} \mathcal{D}\left(\pi_{\alpha}(x)^{*}\right) & =\bigcap_{x \in \mathcal{M}} \mathcal{D}\left(W^{*}(x \otimes 1)^{*} W\right) \\
& =\bigcap_{x \in \mathcal{M}} \mathcal{D}\left(W^{*} \overline{x \otimes 1} W\right)=D(\mathcal{M}, G, \alpha),
\end{aligned}
$$

so that $\mathcal{M}_{\alpha}^{\underset{\alpha}{O^{*}}} G$ is self-adjoint.

Conversely, suppose that $\mathcal{M} \underset{\alpha}{O^{*}} G$ is self-adjoint. Since, $\bigcap_{x \in \mathcal{M}} \mathcal{D}\left((x \otimes 1)^{*}\right)=$ $\bigcap_{x \in \mathcal{M}} \mathcal{D}(\overline{x \otimes 1})$, it follows from Lemma 4.6 , that $\zeta_{0} \otimes \varphi_{\iota} \in \bigcap_{x \in \mathcal{M}} \mathcal{D}(\overline{x \otimes 1})$, for all $\zeta_{0} \in \mathcal{D}^{*}(\mathcal{M})$ and for all indices $\iota$. This implies $\zeta_{0} \in \bigcap_{x \in \mathcal{M}} \mathcal{D}(\bar{x})=\mathcal{D}$, therefore $\mathcal{M}$ is self-adjoint. This completes the proof.

Let $\widehat{1}$ denote the identity operator on $L^{2}(\widehat{G})$ and let $\widehat{W}$ be the unitary operator $\mathbb{I} \bar{\otimes} m_{p} \bar{\otimes} \widehat{1}$ on the Hilbert space $\mathcal{H} \bar{\otimes} L^{2}(G) \bar{\otimes} L^{2}(\widehat{G})=L^{2}(G, \mathcal{H}) \bar{\otimes} L^{2}(\widehat{G})$. Then $(\widehat{W} \zeta)(p)=\left(\mathbb{I} \bar{\otimes} m_{p}\right) \zeta(p)$ for $p \in \widehat{G}$ and $\zeta \in D_{0}(\mathcal{M} \rtimes G, \widehat{G}, \widehat{\alpha})$. Let also $\widehat{\lambda}_{p}$ be the unitary operator on $L^{2}(\widehat{G})$ defined by $\left(\widehat{\lambda}_{p} g\right)(q)=g\left(p^{-1} q\right)$ and $\widehat{\lambda}(p)$ the unitary operator $\mathbb{I} \bar{\otimes} 1 \bar{\otimes} \widehat{\lambda}_{p}$ on $\mathcal{H} \bar{\otimes} L^{2}(G) \bar{\otimes} L^{2}(\widehat{G})$. Using Lemma 4.1 and Theorem 4.7 for the covariant system $\left(\mathcal{M} \underset{\alpha}{O^{*}} G, \widehat{G}, \widehat{\alpha}\right)$ instead of $(\mathcal{M}, G, \alpha)$, we obtain the following result.

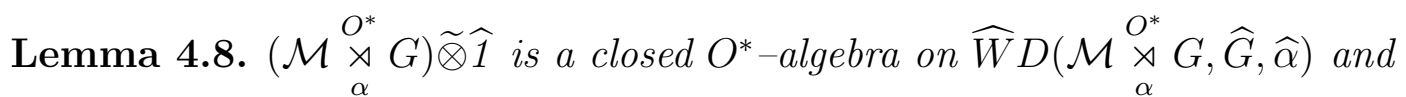

$$
\begin{aligned}
& \pi_{\widehat{\alpha}}\left(\pi_{\alpha}(x)\right)=\widehat{W}^{*}\left(\pi_{\alpha}(x) \widetilde{\otimes} \widehat{1}\right) \widehat{W}, \\
& \pi_{\widehat{\alpha}}(\lambda(s)) \zeta=\widehat{W}^{*}(\lambda(s) \bar{\otimes} \widehat{1}) \widehat{W} \zeta, \\
& \widehat{\lambda}(p) \zeta=\widehat{W}^{*}\left(\mathbb{I} \bar{\otimes} m_{p} \bar{\otimes} \lambda_{p}\right) \widehat{W} \zeta,
\end{aligned}
$$

for all $x \in \mathcal{M}, s \in G, p \in \widehat{G}$ and $\zeta \in D\left(\mathcal{M} \underset{\alpha}{\underset{\alpha}{O^{*}}} G, \widehat{G}, \widehat{\alpha}\right)$. Hence, $\left(\mathcal{M} \underset{\alpha}{\mathcal{O}_{\alpha}^{*}} G\right) \underset{\widehat{\alpha}}{\stackrel{O^{*}}{\rtimes}}$ $\widehat{G}$ is spatially isomorphic to the closed $O^{*}$-algebra generated by restrictions to $\widehat{W} D(\mathcal{M} \rtimes G, \widehat{G}, \widehat{\alpha})$ of the operators $\left\{\pi_{\alpha}(x) \widetilde{\otimes} \widehat{1}, \mathbb{I} \bar{\otimes} \lambda_{s} \bar{\otimes} \widehat{1}, \mathbb{I} \bar{\otimes} m_{p} \bar{\otimes} \widehat{\lambda}_{p} ; x \in \mathcal{M}, s \in\right.$ $G, p \in \widehat{\alpha}\}$.

Lemma 4.9. For any $f \in \mathcal{C}_{c}(G \times G)$, put

$$
(V f)(s, t):=f(s t, t), \forall s, t \in G \text {. }
$$

Then $V$ may be extended to a unitary operator on $L^{2}(G \times G)$ with the following properties:

(1) $V \mathcal{C}_{c}(G \times G) \subset \mathcal{C}_{c}(G \times G)$,

(2) $\left(V^{*} f\right)(s, t)=f\left(s t^{-1}, t\right), \forall s, t \in G$,

(3) $V^{*}\left(m_{p} \bar{\otimes} m_{p}\right) V=m_{p} \bar{\otimes} 1, \forall p \in \widehat{G}$,

(4) $V^{*}\left(\lambda_{s} \bar{\otimes} 1\right) V=\lambda_{s} \bar{\otimes} 1, \forall s \in G$,

(5) $(\mathbb{I} \bar{\otimes} V)\left(W D(\mathcal{M}, G, \alpha) \otimes L^{2}(G)\right) \subset \mathcal{D}\left(\overline{L_{x} \otimes 1}\right)$ and

$(\mathbb{I} \bar{\otimes} V)\left(L_{x} \otimes 1\right)(W \xi \otimes h)=\overline{L_{x} \otimes 1}(\mathbb{I} \bar{\otimes} V)(W \xi \otimes h)$, 
for all $x \in \mathcal{M}, \xi \in D(\mathcal{M}, G, \alpha)$ and $h \in L^{2}(G)$.

(6) $(\mathbb{I} \bar{\otimes} V)^{*}\left(W D(\mathcal{M}, G, \alpha) \otimes L^{2}(G)\right) \subset \mathcal{D}\left(\overline{L_{x} \otimes 1}\right)$, and $(\mathbb{I} \bar{\otimes} V)^{*}\left(L_{x} \otimes 1\right)(W \xi \otimes h)=\left(\overline{L_{x} \otimes 1}\right)(\mathbb{I} \bar{\otimes} V)^{*}(W \xi \otimes h)$, for all $x \in \mathcal{M}, \xi \in D(\mathcal{M}, G, \alpha)$ and $h \in L^{2}(G)$.

Proof. The statements, (1)-(4) are almost trivial.

(5) We define a unitary operator $\widehat{V}$ on $L^{2}(G, \mathcal{H}) \bar{\otimes} L^{2}(G)$, as follows:

$[\widehat{V}(\xi \otimes h)](s, t):=\xi(s t) h(t), \forall s, t \in G, \xi \in L^{2}(G, \mathcal{H})$ and $h \in L^{2}(G)$.

Now, since

$$
\begin{aligned}
\left\|\widehat{V}(\xi \otimes h)-(\mathbb{I} \otimes V)\left(\eta_{0} \otimes g \otimes h\right)\right\|^{2} & =\iint\left\|\xi(s t) h(t)-\eta_{0} g(s t) h(t)\right\|^{2} d s d t \\
& =\|h\|^{2}\left\|\xi-\eta_{0} \otimes g\right\|^{2},
\end{aligned}
$$

for all $\xi \in L^{2}(G, \mathcal{H}), h \in L^{2}(G), \eta_{0} \in \mathcal{D}, g \in \mathcal{C}_{c}(G)$ and since $\mathcal{D} \otimes \mathcal{C}_{c}(G)$ is dense in $L^{2}(G, \mathcal{H})$, we obtain

$$
\widehat{V}(\xi \otimes h)=(\mathbb{I} \bar{\otimes} V)(\xi \otimes h), \forall \xi \in L^{2}(G, \mathcal{H}), \quad \text { and } h \in L^{2}(G) .
$$

Similarly we can show that

$$
\left[(\mathbb{I} \bar{\otimes} V)^{*}(\xi \otimes h)\right](s, t)=\xi\left(s t^{-1}\right) h(t),
$$

for all $s, t \in G, \xi \in L^{2}(G, \mathcal{H})$ and $h \in L^{2}(G)$. Note now that if $\left\{\varphi_{n}\right\}$ is an orthonormal basis in $L^{2}(G)$, then the correspondence

$$
\bigoplus_{n=1}^{\infty} L^{2}(G, \mathcal{H}) \ni\left(\xi_{n}\right) \mapsto \sum_{n=1}^{\infty} \xi_{n} \otimes \varphi_{n} \in L^{2}(G, \mathcal{H}) \bar{\otimes} L^{2}(G)
$$

establishes a canonical identification between the direct sum $\bigoplus_{n=1}^{\infty} L^{2}(G, \mathcal{H})$ of the spaces $L^{2}(G, \mathcal{H})$ and the tensor product Hilbert space $L^{2}(G, \mathcal{H}) \bar{\otimes} L^{2}(G)$.

Similarly to the proof of Lemma 4.6, one obtains

$$
\begin{aligned}
\mathcal{D}\left(\left(L_{x} \otimes 1\right)^{*}\right)=\{ & \sum_{n=1}^{\infty} \xi_{n} \otimes \varphi_{n} ; \xi_{n} \in \mathcal{D}\left(L_{x}^{*}\right), \forall n \in \mathbb{N} \text { and } \\
& \left.\sum_{n=1}^{\infty}\left\|L_{x}^{*} \xi_{n}\right\|^{2}<\infty\right\}, x \in \mathcal{M},
\end{aligned}
$$

and

$$
\left(L_{x} \otimes 1\right)^{*}\left(\sum_{n=1}^{\infty} \xi_{n} \otimes \varphi_{n}\right)=\sum_{n=1}^{\infty} L_{x}^{*} \xi_{n} \otimes \varphi_{n}, \sum_{n=1}^{\infty} \xi_{n} \otimes \varphi_{n} \in \mathcal{D}\left(\left(L_{x} \otimes 1\right)^{*}\right) .
$$

Moreover,

$$
\begin{aligned}
\left(\left(L_{x}^{*} \xi\right)\left(s t^{-1}\right) \mid\right. & (W \eta)(s)) \\
& =\sum_{k}\left(\varphi_{k}\left(s t^{-1}\right) x^{*} \xi_{k}^{O} \mid(W \eta)(s)\right) \\
& =\sum_{k}\left(\varphi_{k}\left(s t^{-1}\right) \xi_{k}^{O} \mid x(W \eta)(s)\right)=\left(\xi\left(s t^{-1}\right) \mid x(W \eta)(s)\right)
\end{aligned}
$$


for all $\xi=\sum_{k} \xi_{k}^{0} \otimes \varphi_{k} \in \mathcal{D}\left(L_{x}^{*}\right), \eta \in D(\mathcal{M}, G, \alpha)$ and $s, t \in G$, which by $(4.3)$ and Lemma 4.6 implies that

$$
\begin{aligned}
\left(\left(L_{x} \otimes 1\right)^{*} \sum_{n} \xi_{n} \otimes \varphi_{n} \mid\right. & (\mathbb{I} \bar{\otimes} V)(W \xi \otimes h)) \\
& =\sum_{n}\left(L_{x}^{*} \xi_{n} \otimes \varphi_{n} \mid(\mathbb{I} \bar{\otimes} V)(W \xi \otimes h)\right) \\
& =\sum_{n} \iint\left(\left(L_{x}^{*} \xi_{n}\right)(s) \varphi_{n}(t) \mid(W \xi)(s t) h(t)\right) d s d t \\
& =\sum_{n} \int \varphi_{n}(t) \overline{h(t)} d t \int\left(\left(L_{x}^{*} \xi_{n}\right)(s) \mid(W \xi)(s t)\right) d s \\
& =\sum_{n} \int \varphi_{n}(t) \overline{h(t)} d t \int\left(L_{x}^{*} \xi_{n}\left(s t^{-1}\right) \mid(W \xi)(s)\right) d s \\
& =\sum_{n} \int \varphi_{n}(t) \overline{h(t)} d t \int\left(\xi_{n}\left(s t^{-1}\right) \mid x(W \xi)(s)\right) d s \\
& =\left((\mathbb{I} \otimes V)\left(\sum_{n} \xi_{n} \otimes \varphi_{n}\right) \mid\left(L_{x} \otimes 1\right)(W \xi \otimes h)\right)
\end{aligned}
$$

for all $x \in \mathcal{M}, \xi \in D(\mathcal{M}, G, \alpha), \sum_{n} \xi_{n} \otimes \varphi_{n} \in \mathcal{D}\left(\left(L_{x} \otimes 1\right)^{*}\right)$ and $h, g \in L^{2}(G)$, which yields that $(\mathbb{I} \bar{\otimes} V)(W \xi \otimes h) \in \mathcal{D}\left(\overline{L_{x} \otimes 1}\right)$ and

$$
\left(\overline{L_{x} \otimes 1}\right)(\mathbb{I} \bar{\otimes} V)(W \xi \otimes h)=(\mathbb{I} \bar{\otimes} V)\left(L_{x} \otimes 1\right)(W \xi \otimes h) .
$$

(6) This is proved in a similar way to (5) by using (4.4), (4.5), (4.6).

We now define a unitary operator

$$
\begin{gathered}
U: \mathcal{H} \bar{\otimes} L^{2}(G) \bar{\otimes} L^{2}(\widehat{G}) \mapsto \mathcal{H} \bar{\otimes} L^{2}(G) \bar{\otimes} L^{2}(G), \quad \text { by } \\
U:=(I \bar{\otimes} V)^{*}(I \bar{\otimes} 1 \bar{\otimes} \mathfrak{F})(W \bar{\otimes} \widehat{1}) \widehat{W},
\end{gathered}
$$

where $\mathfrak{F}$ is the Fourier transform of $L^{2}(\widehat{G})$ onto $L^{2}(G)$.

By Theorem 4.7, $L_{\mathcal{M}} \otimes 1=(\mathcal{M} \widetilde{\otimes} 1) \otimes 1$ is an $O^{*}$-algebra on $W D(\mathcal{M}, G, \alpha) \otimes$ $\mathfrak{F} L^{2}(\widehat{G})$ in $\mathcal{H} \bar{\otimes} L^{2}(G) \bar{\otimes} L^{2}(G)$, whose closure is denoted by $L_{\mathcal{M}} \widetilde{\otimes} 1$. That is,

$$
\begin{gathered}
\mathcal{D}\left(L_{\mathcal{M}} \widetilde{\otimes} 1\right)=\bigcap_{x \in \mathcal{M}} \mathcal{D}\left(\overline{L_{x} \otimes 1}\right):=W D(\mathcal{M}, G, \alpha) \widetilde{\otimes} L^{2}(G), \\
L_{x} \widetilde{\otimes} 1:=\overline{L_{x} \otimes 1}\left\lceil_{\mathcal{D}\left(L_{\mathcal{M}} \widetilde{\otimes} 1\right)}, x \in \mathcal{M} .\right.
\end{gathered}
$$

Since, $\overline{L_{x} \otimes 1}=\overline{(x \widetilde{\otimes} 1) \otimes 1}=\overline{x \otimes(1 \otimes 1)}$, for every $x \in \mathcal{M}$, we may denote $L_{x} \widetilde{\otimes} 1$ by $x \widetilde{\otimes} 1 \widetilde{\otimes} 1$ and $L_{\mathcal{M}} \widetilde{\otimes} 1$ by $\mathcal{M} \widetilde{\otimes} 1 \widetilde{\otimes} 1$.

In this regard, we state the following

Lemma 4.10. We have

$$
\mathcal{D}(\mathcal{M} \widetilde{\otimes} 1 \widetilde{\otimes} 1):=\mathcal{D}\left(L_{\mathcal{M}} \widetilde{\otimes} 1\right)=W D(\mathcal{M}, G, \alpha) \widetilde{\otimes} L^{2}(G)=U D\left(\mathcal{M}_{\alpha}^{O^{*}} G, \widehat{G}, \widehat{\alpha}\right) .
$$


Moreover, $\pi_{\widehat{\alpha}}\left(\pi_{\alpha}(x)\right) \zeta=U^{*}(x \widetilde{\otimes} 1 \widetilde{\otimes} 1) U \zeta, \pi_{\widehat{\alpha}}(\lambda(s)) \zeta=U^{*}\left(w_{s} \bar{\otimes} \lambda_{s} \bar{\otimes} 1\right) U \zeta$, and $\lambda(p) \zeta=U^{*}\left(\mathbb{I} \bar{\otimes} m_{p} \bar{\otimes} 1\right) U \zeta$, for all $x \in \mathcal{M}, s \in G, p \in \widehat{G}$ and $\zeta \in D\left(\mathcal{M}_{\alpha}^{\text {X }_{\alpha}^{*}} G, \widehat{G}, \widehat{\alpha}\right)$.

Proof. We first show that

$$
\begin{gathered}
\widehat{W} D_{0}\left(\mathcal{M} \underset{\alpha}{\stackrel{O^{*}}{\alpha}} G, \widehat{G}, \widehat{\alpha}\right)=D_{0}\left(\mathcal{M} \underset{\alpha}{O_{\alpha}^{*}} G, \widehat{G}, \widehat{\alpha}\right) \text { and } \\
\pi_{\widehat{\alpha}}\left(\pi_{\alpha}(x)\right) \zeta=\widehat{W}^{*} \pi_{\widehat{\alpha}}\left(\pi_{\alpha}(x)\right) \widehat{W} \zeta, \forall x \in \mathcal{M} \text { and } \zeta \in D_{0}\left(\underset{\mathcal{M}_{\alpha}}{O_{\alpha}^{*}} G, \widehat{G}, \widehat{\alpha}\right) .
\end{gathered}
$$

Indeed, take arbitrary $x \in \mathcal{M}$ and $\zeta \in D_{0}\left(\mathcal{M} \underset{\alpha}{O_{\alpha}^{*}} G, \widehat{G}, \widehat{\alpha}\right)$. By Lemma 4.1,

$$
(\widehat{W} \zeta)(p)=\left(\mathbb{I} \bar{\otimes} m_{p}\right) \zeta(p) \in D(\mathcal{M}, G, \alpha), \forall p \in \widehat{G} .
$$

Again by Lemma 4.1, for every $p_{0} \in \widehat{G}$ fixed, we obtain

$$
\begin{aligned}
& \left\|\pi_{\alpha}(x)(\widehat{W} \zeta)(p)-\pi_{\alpha}(x)(\widehat{W} \zeta)\left(p_{0}\right)\right\|=\left\|\left(\mathbb{I} \bar{\otimes} m_{p}\right) \pi_{\alpha}(x) \zeta(p)-\left(\mathbb{I} \bar{\otimes} m_{p_{0}}\right) \pi_{\alpha}(x) \zeta\left(p_{0}\right)\right\| \\
& \quad \leqq\left\|\pi_{\alpha}(x) \zeta(p)-\pi_{\alpha}(x) \zeta\left(p_{0}\right)\right\|+\left\|\left(\mathbb{I} \bar{\otimes} m_{p}\right) \pi_{\alpha}(x) \zeta\left(p_{0}\right)-\left(I \bar{\otimes} m_{p_{0}}\right) \pi_{\alpha}(x) \zeta\left(p_{0}\right)\right\| .
\end{aligned}
$$

Hence, (4.1) implies that the correspondence

$$
\widehat{G} \ni p \longmapsto \widehat{\alpha_{p^{-1}}}\left(\pi_{\alpha}(x)\right) \zeta(p) \in L^{2}(G, \mathcal{H}) \text {, is continuous }, \forall x \in \mathcal{M} \text {. }
$$

Thus, $\widehat{W} \zeta \in D_{0}\left(\mathcal{M}_{\alpha}^{O_{\alpha}^{*}} G, \widehat{G}, \widehat{\alpha}\right)$. Similarly we obtain that $\widehat{W}^{*} \zeta \in D_{0}\left(\mathcal{M}_{\alpha}^{O_{\alpha}^{*}} G, \widehat{G}, \widehat{\alpha}\right)$. Therefore the statement (4.7) holds. Now by Lemma 4.1, for each $x \in \mathcal{M}$ and $\zeta \in D_{0}\left(\mathcal{M} \underset{\alpha}{O^{*}} G, \widehat{G}, \widehat{\alpha}\right)$, we have

$$
\left[\widehat{W}^{*} \pi_{\widehat{\alpha}}\left(\pi_{\alpha}(x)\right) \widehat{W} \zeta\right](p)=\pi_{\widehat{\alpha}}\left(\pi_{\alpha}(x)\right) \zeta(p), \forall p \in \widehat{G},
$$

which implies (4.8). Furthermore, (4.2), (4.7) and (4.8) yield that

$$
\begin{gathered}
\widehat{W} D\left(\mathcal{M}_{\alpha}^{O_{\alpha}^{*}} G, \widehat{G}, \widehat{\alpha}\right)=D\left(\mathcal{M} \underset{\alpha}{\underset{\alpha}{O^{*}}} G, \widehat{G}, \widehat{\alpha}\right), \\
\pi_{\widehat{\alpha}}\left(\pi_{\alpha}(x)\right) \zeta=\widehat{W}^{*} \pi_{\widehat{\alpha}}\left(\pi_{\alpha}(x)\right) \widehat{W} \zeta, x \in \mathcal{M}, \zeta \in D\left(\mathcal{M} \underset{\alpha}{O_{\alpha}^{*}} G, \widehat{G}, \widehat{\alpha}\right) .
\end{gathered}
$$

Yet by (5) and (6) of Lemma 4.9 and by (4.2) we conclude that

$$
\begin{gathered}
(I \bar{\otimes} V)\left(W D(\mathcal{M}, G, \alpha) \widetilde{\otimes} L^{2}(G)\right) \subset \mathcal{D}(\mathcal{M} \widetilde{\otimes} 1 \widetilde{\otimes} 1)=W D(\mathcal{M}, G, \alpha) \widetilde{\otimes} L^{2}(G) \text { and } \\
(I \bar{\otimes} V)^{*}\left(W D(\mathcal{M}, G, \alpha) \widetilde{\otimes} L^{2}(G)\right) \subset W D(\mathcal{M}, G, \alpha) \widetilde{\otimes} L^{2}(G),
\end{gathered}
$$

which implies that

$$
\begin{aligned}
U D\left(\mathcal{M} \underset{\alpha}{O_{\alpha}^{*}} G, \widehat{G}, \widehat{\alpha}\right) & =(\mathbb{I} \bar{\otimes} V)^{*}\left(W D(\mathcal{M}, G, \alpha) \widetilde{\otimes} L^{2}(G)\right) \\
& =W D(\mathcal{M}, G, \alpha) \widetilde{\otimes} L^{2}(G) .
\end{aligned}
$$


Continuing, we consider $x \in \mathcal{M}, \xi \in D_{0}(\mathcal{M}, G, \alpha)$ and $h \in \mathcal{C}_{c}(\widehat{G})$, so that we have

$$
\begin{aligned}
\pi_{\widehat{\alpha}} & \left(\pi_{\alpha}(x)\right)(\xi \otimes h) \\
& =\pi_{\alpha}(x)(\xi \otimes h) \quad(\text { by }(4.1)) \\
& =W^{*} x \widetilde{\otimes} 1 W(\xi \otimes h) \quad(\text { by Lemma 4.5) } \\
& =(W \bar{\otimes} \widehat{1})^{*}(x \widetilde{\otimes} 1 \otimes \widehat{1})(W \bar{\otimes} \widehat{1})(\xi \otimes h) \\
& =(W \bar{\otimes} 1)^{*}(I \bar{\otimes} 1 \bar{\otimes} \mathfrak{F})^{*}(x \widetilde{\otimes} 1 \otimes 1)(I \bar{\otimes} 1 \bar{\otimes} \mathfrak{F})(W \bar{\otimes} \widehat{1})(\xi \otimes h) \\
& =(W \bar{\otimes} 1)^{*}(I \bar{\otimes} 1 \bar{\otimes} \mathfrak{F})^{*}(I \bar{\Phi} V)(x \widetilde{\otimes} 1 \widetilde{\otimes} 1)(I \bar{\otimes} V)^{*}(I \bar{\otimes} 1 \bar{\otimes} \mathfrak{F})(W \bar{\otimes} \widehat{1})(\xi \otimes h),
\end{aligned}
$$

where in the last equality we applied Lemma 4.9. This, together with (4.2) and Lemma 4.9 imply that

$$
\pi_{\widehat{\alpha}}\left(\pi_{\alpha}(x)\right) \zeta=(W \bar{\otimes} 1)^{*}(I \bar{\otimes} 1 \bar{\otimes} \mathfrak{F})^{*}(I \bar{\otimes} V)(x \widetilde{\otimes} 1 \widetilde{\otimes} 1)(I \bar{\otimes} V)^{*}(I \bar{\otimes} 1 \bar{\otimes} \mathfrak{F})(W \bar{\otimes} \widehat{1}) \zeta,
$$

for all $\zeta \in D\left(\mathcal{M} \underset{\alpha}{O_{\alpha}^{*}} G, \widehat{G}, \widehat{\alpha}\right)$. Furthermore, by (4.9) and (4.10), we obtain

$$
\begin{aligned}
& \widehat{W} \pi_{\widehat{\alpha}}\left(\pi_{\alpha}(x)\right) \zeta=\pi_{\widehat{\alpha}}\left(\pi_{\alpha}(x)\right) \widehat{W} \zeta \\
& \quad=(W \bar{\otimes} 1)^{*}(I \bar{\otimes} 1 \bar{\otimes} \mathfrak{F})^{*}(I \bar{\otimes} V)(x \widetilde{\otimes} 1 \widetilde{\otimes} 1)(I \bar{\otimes} V)^{*}(I \bar{\otimes} 1 \bar{\otimes} \mathfrak{F})(W \bar{\otimes} \widehat{1}) \widehat{W} \zeta .
\end{aligned}
$$

Hence, we have

$$
\pi_{\widehat{\alpha}}\left(\pi_{\alpha}(x)\right) \zeta=U^{*}(x \widetilde{\otimes} 1 \widetilde{\otimes} 1) U \zeta, \forall \zeta \in D\left(\mathcal{M} \underset{\alpha}{O_{\alpha}^{*}} G, \widehat{G}, \widehat{\alpha}\right) .
$$

Similarly,

$$
\pi_{\widehat{\alpha}}(\lambda(s))=\widehat{W}^{*}\left(I \bar{\otimes} \lambda_{s} \bar{\otimes} 1\right) \widehat{W}=U^{*}\left(w_{s} \bar{\otimes} \lambda_{s} \bar{\otimes} 1\right) U .
$$

Using now that $W$ and $\mathbb{I} \bar{\otimes} m_{p}$ commute and that $\mathfrak{F} \lambda_{p} \mathfrak{F}^{*}=m_{p}$, for every $p \in \widehat{G}$, we obtain

$$
\begin{aligned}
\lambda(p) & =\widehat{W}^{*}\left(I \bar{\otimes} m_{p} \bar{\otimes} \lambda_{p}\right) \widehat{W} \\
& =\widehat{W}^{*}(W \bar{\otimes} \hat{1})^{*}\left(I \bar{\otimes} m_{p} \bar{\otimes} \lambda_{p}\right)(W \bar{\otimes} \widehat{1}) \widehat{W} \\
& =\widehat{W}^{*}(W \bar{\otimes} \widehat{1})^{*}(I \bar{\otimes} 1 \bar{\otimes} \mathfrak{F})^{*}\left(I \bar{\otimes} m_{p} \bar{\otimes} m_{p}\right)(I \bar{\otimes} 1 \bar{\otimes} \mathfrak{F})(W \bar{\otimes} \widehat{1}) \widehat{W} \\
& \left.=U^{*}\left(I \bar{\otimes} m_{p} \bar{\otimes} 1\right) U \text { (by Lemma } 4.9(3)\right) .
\end{aligned}
$$

This completes the proof.

By Lemmas 4.9 and 4.10 we have the following

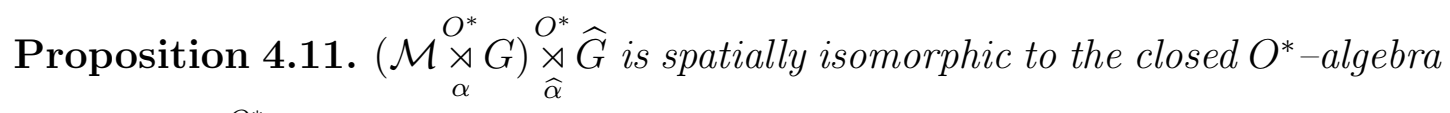
on $U D\left(\mathcal{M} \underset{\alpha}{O^{*}} G, \widehat{G}, \widehat{\alpha}\right)$ generated by restrictions of the operators

$$
\left\{x \widetilde{\otimes} 1 \widetilde{\otimes} 1, w_{s} \bar{\otimes} \lambda_{s} \bar{\otimes} 1, I \bar{\otimes} m_{p} \bar{\otimes} 1 ; x \in \mathcal{M}, s \in G, p \in \widehat{G}\right\} .
$$


Let now $\mathfrak{A}$ be the closed $O^{*}$-algebra on $W D(\mathcal{M}, G, \alpha)$ generated by the operators $\left\{x \widetilde{\otimes} 1, w_{s} \bar{\otimes} \lambda_{s}, \mathbb{I} \bar{\otimes} m_{p} ; x \in \mathcal{M}, s \in G, p \in \widehat{G}\right\}$. Then

$$
(\mathcal{M} \underset{\alpha}{\rtimes} G) \underset{\widehat{\alpha}}{O^{*}} \underset{O^{*}}{\rtimes} \widehat{G}=U^{*}(\mathfrak{A} \widetilde{\otimes} 1) U .
$$

Thus, we have the following

Theorem 4.12. $\left(\mathcal{M}_{\alpha}^{\underset{\alpha}{O^{*}}} G\right) \underset{\widehat{\alpha}}{\stackrel{O^{*}}{\rtimes}} \widehat{G}$ is spatially isomorphic to the closed $O^{*}$-algebra $\mathfrak{A} \widetilde{\otimes} 1$, where $\mathfrak{A}$ is a closed $O^{*}$-algebra on $W D(\mathcal{M}, G, \alpha)$, generated by the operators

$$
\left\{x \widetilde{\otimes} 1, w_{s} \bar{\otimes} \lambda_{s}, \mathbb{I} \bar{\otimes} m_{p} ; x \in \mathcal{M}, s \in G, p \in \widehat{G}\right\} .
$$

Now by (4.11) the map

$$
\left(\mathcal{M} \underset{\alpha}{\stackrel{O^{*}}{\rtimes}} G \underset{\widehat{\alpha}}{\stackrel{O^{*}}{\rtimes}} \widehat{G} \longrightarrow \mathfrak{A}: U^{*}(X \widetilde{\otimes} 1) U \longmapsto X,\right.
$$

is a $*$-isomorphism onto. Hence, we obtain

Theorem 4.13. $\left(\mathcal{M} \underset{\alpha}{\underset{\alpha}{O^{*}} G} \underset{\widehat{\alpha}}{\stackrel{O^{*}}{\rtimes}} \widehat{G}\right.$ is isomorphic to the closed $O^{*}$-algebra $\mathfrak{A}$ on the domain $W D(\mathcal{M}, G, \alpha)$, generated by the operators

$$
\left\{x \widetilde{\otimes} 1, w_{s} \bar{\otimes} \lambda_{s}, \mathbb{I} \bar{\otimes} m_{p} ; x \in \mathcal{M}, s \in G, p \in \widehat{G}\right\} .
$$

For the $G W^{*}$-crossed product $\left(\mathcal{M} \underset{\alpha}{\underset{O^{*}}{\rtimes}} G\right) \underset{\widehat{\alpha}}{\stackrel{G W^{*}}{\rtimes}} \widehat{G}$ we now conclude

Proposition 4.14. Suppose that $\left(\mathcal{M} \underset{\alpha}{\stackrel{O^{*}}{\rtimes}} G\right)_{w}^{\prime} D(\mathcal{M}, G, \alpha) \subset D(\mathcal{M}, G, \alpha)$, which holds in particular, if $\mathcal{M}$ is self-adjoint. Then the $G W^{*}$ - $\operatorname{crossed} \operatorname{product}\left(\mathcal{M} \underset{\alpha}{\mathrm{O}^{*}}\right.$ $G) \underset{\widehat{\alpha}}{G W^{*}} \widehat{G}$ of $\mathcal{M} \underset{\alpha}{\stackrel{O^{*}}{\rtimes}} G$ by the action $\widehat{\alpha}$ of $\widehat{G}$ is well-defined. Furthermore, if $\mathcal{M}$ is a regular $G W^{*}$-algebra, then $\left(\mathcal{M} \underset{\alpha}{\underset{\alpha}{O^{*}}} G\right) \underset{\widehat{\alpha}}{\stackrel{G W^{*}}{\rtimes}} \widehat{G}$ is spatially isomorphic to the $G W^{*}$-algebra

$$
\left(\left(\mathcal{M}_{w}^{\prime}\right)^{\prime} \underset{W D(\mathcal{M}, G, \alpha)}{G W^{*}} \mathcal{B}\left(L^{2}(G)\right)\right) \widetilde{\otimes} 1
$$

and it is isomorphic to the $G W^{*}$-tensor product $\left(\mathcal{M}_{w}^{\prime}\right)^{\prime} \underset{W D(\mathcal{M}, G, \alpha)}{G W^{*}} \mathcal{B}\left(L^{2}(G)\right)$.

Proof. By Theorem 3.5, the assumption $\left(\mathcal{M} \underset{\alpha}{O_{\alpha}^{*}} G\right)_{w}^{\prime} D(\mathcal{M}, G, \alpha) \subset D(\mathcal{M}, G, \alpha)$ guarantees that $\left(\mathcal{M} \underset{\alpha}{\stackrel{O^{*}}{\rtimes}} G\right) \underset{\widehat{\alpha}}{\underset{\rtimes}{\rtimes}} \widehat{G} \widehat{G}$ is well-defined. Furthermore, if $\mathcal{M}$ is selfadjoint, $\mathcal{M} \underset{\alpha}{O^{*}} G$ is self-adjoint by Theorem 4.7. This implies that the assumption $\left(\mathcal{M} \underset{\alpha}{O^{*}} G\right)_{w}^{\prime} D(\mathcal{M}, G, \alpha) \subset D(\mathcal{M}, G, \alpha)$ is satisfied. 
Suppose now that $\mathcal{M}$ is regular. Then it is easily shown that $\left(\left(\mathcal{M} \underset{\alpha}{O_{\alpha}^{*}} G\right)_{w}^{\prime}\right)^{\prime}=$ $\left(\mathcal{M}_{w}^{\prime}\right)^{\prime} \rtimes G$. Moreover, similarly to (4.11) one obtains that

$$
\begin{gathered}
\left(\left(\mathcal{M}_{w}^{\prime}\right)^{\prime} \underset{\alpha}{\rtimes} G\right) \underset{\widehat{\alpha}}{\rtimes} \widehat{G}=U^{*}\left(\mathcal{R} \stackrel{W}{\otimes}^{*} 1\right) U, \text { where } \\
\mathcal{R}=\left\{a \bar{\otimes} 1, w_{s} \bar{\otimes} \lambda_{s}, \mathbb{I} \bar{\otimes} m_{p} ; a \in\left(\mathcal{M}_{w}^{\prime}\right)^{\prime}, s \in G, p \in \widehat{G}\right\}^{\prime \prime} .
\end{gathered}
$$

Since, $\mathcal{R}=\left(\mathcal{M}_{w}^{\prime}\right)^{\prime} \stackrel{W}{*}^{*} \mathcal{B}\left(L^{2}(G)\right)$, by [32, Lemma I.4.9], it follows that

$$
\left(\left(\mathcal{M}_{w}^{\prime}\right)^{\prime} \underset{\alpha}{\rtimes} G\right) \underset{\widehat{\alpha}}{\rtimes} \widehat{G}=U^{*}\left(\left(\mathcal{M}_{w}^{\prime}\right)^{\prime} \stackrel{W^{*}}{\otimes} \mathcal{B}\left(L^{2}(G)\right)^{W^{*}} \otimes 1\right) U,
$$

which implies that

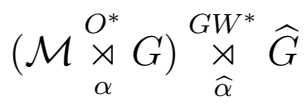

$$
\begin{aligned}
& =U^{*}\left\{X \widetilde{\otimes} 1 \in \mathcal{L}^{\dagger}\left(U D\left(\mathcal{M} \underset{\alpha}{O^{*}} G, \widehat{G}, \widehat{\alpha}\right)\right) ; \bar{X} \eta\left(\mathcal{M}_{w}^{\prime}\right)^{\prime}{ }^{W^{*}} \otimes \mathcal{B}\left(L^{2}(G)\right)\right\} U \\
& =U^{*}\left(\left(\mathcal{M}_{w}^{\prime}\right)^{\prime} \underset{W D(\mathcal{M}, G, \alpha)}{G W^{*}} \mathcal{B}\left(L^{2}(G)\right) \widetilde{\otimes} 1\right) U .
\end{aligned}
$$

Consequently, $\left(\mathcal{M} \underset{\alpha}{\underset{O^{*}}{\rtimes}} G\right) \underset{\widehat{\alpha}}{\stackrel{G W^{*}}{\rtimes}} \widehat{G}$ is spatially isomorphic to the $G W^{*}$-algebra

$$
\left(\left(\mathcal{M}_{w}^{\prime}\right)^{\prime} \underset{W D(\mathcal{M}, G, \alpha)}{G W^{*}} \mathcal{B}\left(L^{2}(G)\right)\right) \widetilde{\otimes} 1 .
$$

This completes the proof.

Lemma 4.15. Suppose that $\mathcal{D}\left[t_{\mathcal{M}}\right]$ is a Fréchet space. Then the following statements hold:

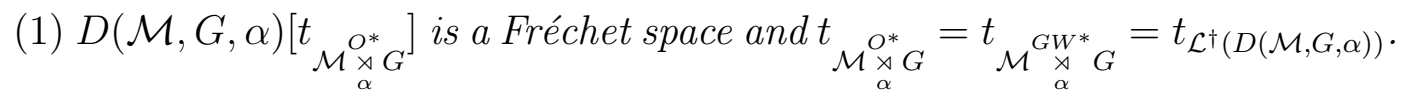

(2) $\widehat{\alpha}$ is a strongly continuous action of $\widehat{G}$ on $\mathcal{M} \underset{\alpha}{G W^{*}} G$.

(3) $D\left(\mathcal{M} \underset{\alpha}{O_{\star}^{*}} G, \widehat{G}, \widehat{\alpha}\right)=D\left(\mathcal{M} \underset{\alpha}{G W^{*}} G, \widehat{G}, \widehat{\alpha}\right)$ and they are Fréchet spaces under

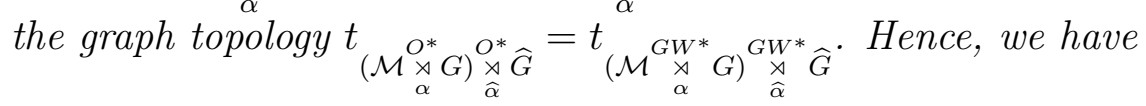

$$
\left(\mathcal{M} \underset{\alpha}{\stackrel{O^{*}}{\rtimes}} G\right) \underset{\widehat{\alpha}}{\stackrel{O^{*}}{\rtimes}} \widehat{G} \subset\left(\mathcal{M} \underset{\alpha}{\stackrel{G W^{*}}{\rtimes}} G\right) \underset{\widehat{\alpha}}{\stackrel{\rightarrow W^{*}}{\rtimes}} \widehat{G} .
$$

Proof. (1) Since, $\mathcal{D}\left[t_{\mathcal{M}}\right]$ is a Fréchet space, there exists a sequence $\left\{a_{n}\right\}$ in $\mathcal{M}$ defining the graph topology $t_{\mathcal{M}}$ such that $0 \leqq a_{1} \leqq a_{2} \leqq \cdots$. Hence, for any fixed element $x$ of $\mathcal{M}$ there exists an element $a_{n}$ and a positive constant $\gamma$ such that

$$
\left\|x \xi_{0}\right\| \leqq \gamma\left\|a_{n} \xi_{0}\right\|, \forall \xi_{0} \in \mathcal{D}
$$

Then

$$
\left\|\pi_{\alpha}(x) \xi\right\|^{2}=\int\left\|x w_{s} \xi(s)\right\|^{2} d s \leqq \gamma^{2} \int\left\|a_{n} w_{s} \xi(s)\right\|^{2} d s=\gamma^{2}\left\|\pi_{\alpha}\left(a_{n}\right) \xi\right\|^{2},
$$


for all $\xi \in D(\mathcal{M}, G, \alpha)$, which implies that $D(\mathcal{M}, G, \alpha)\left[t_{\substack{\mathcal{M}_{\alpha}{ }_{\alpha}^{\circ *} \\ \alpha}}\right]$ is a Fréchet

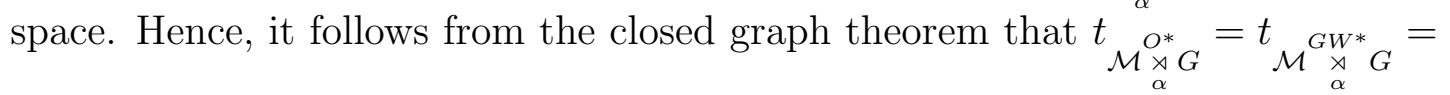
$t_{\mathcal{L}^{\dagger}(D(\mathcal{M}, G, \alpha))}$.

(2) Take an arbitrary $A \in \mathcal{M} \underset{\alpha}{\underset{\sim}{G}} G$. By (1), there exists an element $a_{n}$ and a constant $\gamma$ such that

$$
\|A \xi\| \leqq \gamma\left\|\pi_{\alpha}\left(a_{n}\right) \xi\right\|, \quad \forall \xi \in D(\mathcal{M}, G, \alpha),
$$

which by Lemma 4.1 (2) implies that for any $\xi \in D(\mathcal{M}, G, \alpha)$

$$
\begin{aligned}
& \left\|\widehat{\alpha}_{p^{-1}}(A) \xi-\widehat{\alpha}_{p_{0}^{-1}}(A) \xi\right\|=\left\|\left(\mathbb{I} \bar{\otimes} m_{p}\right)^{*} A\left(\mathbb{I} \bar{\otimes} m_{p}\right) \xi-\left(\mathbb{I} \bar{\otimes} m_{p_{0}}\right) A\left(\mathbb{I} \bar{\otimes} m_{p_{0}}\right) \xi\right\| \\
& \leqq\left\|\left(1 \bar{\otimes} m_{p}\right)^{*} A\left(\left(\amalg \bar{\otimes} m_{p}\right)-\left(\mathbb{I} \bar{\otimes} m_{p_{0}}\right)\right) \xi\right\| \\
& +\left\|\left(\left(I \bar{\otimes} m_{p}\right)^{*}-\left(I \bar{\Phi} \bar{\otimes} m_{p_{0}}\right)^{*}\right) A\left(I \bar{\otimes} m_{p_{0}}\right) \xi\right\| \\
& \leqq \gamma\left\|\pi_{p}\left(a_{n}\right)\left(\left(\mathbb{I} \bar{\otimes} m_{p}\right)-\left(\mathbb{I} \bar{\otimes} m_{p_{0}}\right)\right) \xi\right\| \\
& +\left\|\left(\left(I \bar{\otimes} m_{p}\right)^{*}-\left(I \bar{\otimes} m_{p_{0}}\right)^{*}\right) A\left(I \bar{\otimes} m_{p_{0}}\right) \xi\right\| \\
& =\gamma\left\|\left(\left(\mathbb{I} \bar{\otimes} m_{p}\right)-\left(\mathbb{I I} \bar{\otimes} m_{p_{0}}\right)\right) \pi_{\alpha}\left(a_{n}\right) \xi\right\| \\
& +\left\|\left(\left(1 \bar{\otimes} m_{p}\right)^{*}-\left(\amalg \bar{\otimes} m_{p_{0}}\right)^{*}\right) A\left(1 \bar{\otimes} m_{p_{0}}\right) \xi\right\| \\
& \underset{p \rightarrow p_{0}}{\longrightarrow} 0 \text {. }
\end{aligned}
$$

Hence, $\widehat{\alpha}$ is a strongly continuous action of $\widehat{G}$ on $\mathcal{M}^{G W^{*}} G$.

(3) Take arbitrary $A \in \mathcal{M} \underset{\alpha}{\stackrel{G W^{*}}{\rtimes}} G$ and $\zeta \in D_{0}\left(\mathcal{M} \underset{\alpha}{\stackrel{O^{*}}{\alpha}} G, \widehat{G}, \widehat{\alpha}\right)$. Then from (4.13) it follows that the map $\widehat{G} \ni p \longmapsto A \zeta(p) \in L^{2}(G, \mathbb{R})$ is continuous, which implies that $\zeta \in D_{0}(\mathcal{M} \underset{\alpha}{\stackrel{G}{\rtimes}} G, \widehat{G}, \widehat{\alpha})$. Hence, $D_{0}\left(\mathcal{M} \underset{\alpha}{\stackrel{O^{*}}{\rtimes}} G, \widehat{G}, \widehat{\alpha}\right) \subset D_{0}\left(\mathcal{M} \underset{\alpha}{\stackrel{G W^{*}}{x}} G, \widehat{G}, \widehat{\alpha}\right)$. The converse inclusion is trivial. Thus, we have $D_{0}\left(\mathcal{M} \underset{\alpha}{\underset{\alpha}{O^{*}}} G, \widehat{G}, \widehat{\alpha}\right)=D_{0}\left(\mathcal{M} \underset{\alpha}{\stackrel{G W^{*}}{\rtimes}}\right.$ $G, \widehat{G}, \widehat{\alpha})$. Furthermore, from (4.13) it follows that for any $A \in \mathcal{M} \underset{\alpha}{\stackrel{G}{\rtimes}} G$

$$
\left\|\pi_{\widehat{\alpha}}(A) \zeta\right\| \leqq \gamma\left\|\pi_{\widehat{\alpha}}\left(a_{n}\right) \zeta\right\|, \forall \zeta \in D_{0}\left(\mathcal{M} \underset{\alpha}{O_{\alpha}^{*}} G, \widehat{G}, \widehat{\alpha}\right)=D_{0}\left(\mathcal{M}{\underset{\alpha}{\rtimes}}_{\triangle}^{G W^{*}} G, \widehat{G}, \widehat{\alpha}\right),
$$

which implies that $D\left(\mathcal{M} \stackrel{O}{\rtimes}^{*}, \widehat{G}, \widehat{\alpha}\right)=D\left(\mathcal{M}{ }_{\rtimes}^{G W^{*}} G, \widehat{G}, \widehat{\alpha}\right)$ and that they are

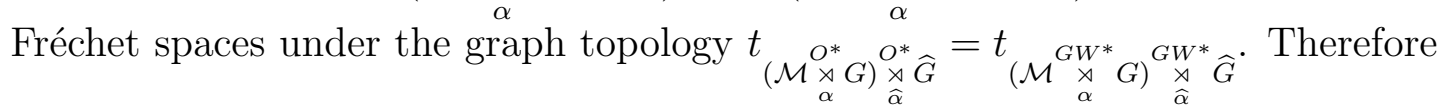

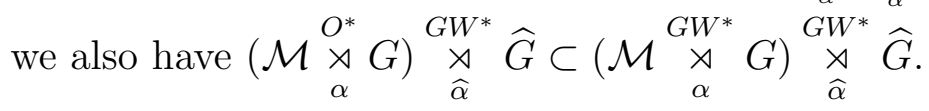

Theorem 4.16. Suppose that $\mathcal{D}\left[t_{\mathcal{M}}\right]$ is a Fréchet space. Then the following statements hold: 
(1) The crossed product $\left(\mathcal{M} \underset{\alpha}{G W^{*}} G\right) \underset{\widehat{\alpha}}{G W^{*}} \widehat{G}$ is spatially isomorphic to the $G W^{*}-$ algebra $\left(\left(\mathcal{M}_{w}^{\prime}\right)^{\prime} \underset{W D(\mathcal{M}, G, \alpha)}{G W^{*}} \mathcal{B}\left(L^{2}(G)\right) \widetilde{\otimes} 1\right.$ and isomorphic to the $G W^{*}$-tensor product $\left(\mathcal{M}_{w}^{\prime}\right)^{\prime} \underset{W D(\mathcal{M}, G, \alpha)}{G W^{*}} \mathcal{B}\left(L^{2}(G)\right)$.

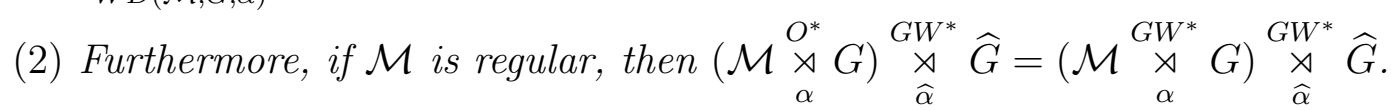
Proof. By Lemma 4.15 and similarly to (4.12), one obtains

$$
\left(\mathcal{M} \underset{\alpha}{G W^{*}} G\right) \underset{\widehat{\alpha}}{G W^{*}} \widehat{G}=U^{*}\left(\left(\left(\mathcal{M}_{w}^{\prime}\right)^{\prime} \underset{W D(\mathcal{M}, G, \alpha)}{\stackrel{G W^{*}}{\otimes}} \mathcal{B}\left(L^{2}(G)\right)\right) \widetilde{\otimes} 1\right) U
$$

which implies the statement (1).

The statement (2) follows from Lemma 4.15 and Proposition 4.14.

Throughout the rest of this section, we suppose that $\mathcal{D}\left[t_{\mathcal{M}}\right]$ is a Fréchet space.

We denote by $\widetilde{\alpha}$ the dual action of $\widehat{\alpha}$, and we call it bidual action of $G$ on the crossed product $(\mathcal{M} \underset{\alpha}{\stackrel{G}{\rtimes}} G) \underset{\widehat{\alpha}}{\underset{x}{x}} \widehat{G W^{*}} \widehat{G}$. Then $\widetilde{\alpha}$ is implemented by the unitaries $\mathbb{I} \bar{\otimes} 1 \bar{\otimes} \widehat{m_{s}}, s \in G$, where $\widehat{m_{s}}$ is defined on $L^{2}(\widehat{G})$ by $\left(\widehat{m_{s}} h\right)(p)=\overline{<s, p>h}(p)$, $h \in L^{2}(\widehat{G}), p \in \widehat{G}$. We show that $\widetilde{\alpha}$ is transformed under the isomoprhism $\gamma$ between $(\mathcal{M} \underset{\alpha}{\underset{\alpha}{\hookrightarrow}} G) \underset{\widehat{\alpha}}{\underset{\rtimes}{\rtimes}} \widehat{G W^{*}} \widehat{G}$ and $\left(\mathcal{M}_{w}^{\prime}\right)^{\prime} \underset{D(\mathcal{M}, G, \alpha)}{\stackrel{G W^{*}}{\otimes}} \mathcal{B}\left(L^{2}(G)\right)$ to the action $\theta$ defined by (3.4) in Section 3 .

Since, $\left(I \bar{\otimes} 1 \bar{\otimes} \widehat{m}_{s}\right)^{*}$ commutes with $(W \bar{\otimes} \widehat{1})$ and $\widehat{W}$, we conclude that

$$
\mathfrak{F} \widehat{m}_{s} \mathfrak{F}^{*}=\rho_{s}, \quad V^{*}\left(1 \bar{\otimes} \rho_{s}\right) V=\rho_{s} \otimes \rho_{s} \quad \text { and } \quad W^{*}\left(I \bar{\otimes} \rho_{s}\right) W=w_{s} \bar{\otimes} \rho_{s}, \quad \forall s \in G .
$$

Thus, we obtain $(W \bar{\otimes} 1)^{*} U\left(\mathbb{I} \bar{\otimes} 1 \bar{\otimes} \widehat{m_{s}}\right)^{*}=\left(w_{s} \bar{\otimes} \rho_{s} \bar{\otimes} \rho_{s}\right)^{*}(W \bar{\otimes} 1)^{*} U$, for each $s \in$ $G$, therefore

$$
\begin{aligned}
\widetilde{\alpha}_{s}\left(U^{*}(W \bar{\otimes} 1)\right. & \left.(X \widetilde{\otimes} 1)(W \bar{\otimes} 1)^{*} U\right) \\
& =\left(I \bar{\otimes} 1 \bar{\otimes} \widehat{m_{s}}\right) U^{*}(W \bar{\otimes} 1)(X \widetilde{\otimes} 1)(W \bar{\otimes} 1)^{*} U\left(I \bar{\otimes} 1 \bar{\otimes} \widehat{m_{s}}\right)^{*} \\
& =U^{*}(W \bar{\otimes} 1)\left(w_{s} \bar{\otimes} \rho_{s} \bar{\otimes} \rho_{s}\right)^{*}(X \widetilde{\otimes} 1)\left(w_{s} \bar{\otimes} \rho_{s} \bar{\otimes} \widehat{m_{s}}\right)^{*}(W \bar{\otimes} 1)^{*} U \\
& =U^{*}(W \bar{\otimes} 1)\left(\left(w_{s} \bar{\otimes} \rho_{s}\right) X\left(w_{s} \bar{\otimes} \rho_{s}\right)^{*} \widetilde{\otimes} 1\right)(W \bar{\otimes} 1) U \\
& =U^{*}(W \bar{\otimes} 1)\left(\theta_{s}(X) \widetilde{\otimes} 1\right)(W \bar{\otimes} 1)^{*} U, \forall s \in G .
\end{aligned}
$$

Hence, we have

$$
\gamma\left(\widetilde{\alpha}_{s}(X)\right)=\theta_{s}(\gamma(X)), \forall X \in\left(\mathcal{M} \underset{\alpha}{G W^{*}} G\right) \underset{\widehat{\alpha}}{G W^{*}} \widehat{G} \text { and } s \in G .
$$

We now obtain the following

Proposition 4.17. The bidual action $\widetilde{\alpha}$ of $G$ on $\left(\mathcal{M} \underset{\alpha}{G W^{*}} G\right) \underset{\widehat{\alpha}}{A W^{*}} \widehat{G}$ is transformed

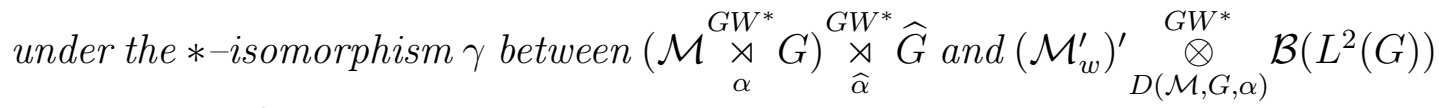
to the action $\theta$. 
Finally we want to know whether the crossed product $\mathcal{M} \underset{\alpha}{\rtimes W^{*}} G$ coincides with the image of $\pi_{\widehat{\alpha}}\left(\mathcal{M}^{G W^{*}} G\right)$ under the $*$-isomorphism $\gamma$. In this regard, we first have

\section{Lemma 4.18.}

$$
\left\{X \in \mathcal{M} \underset{\alpha}{\stackrel{G W^{*}}{\rtimes}} G ; \widehat{\alpha}_{p}(X)=X, \forall p \in \widehat{G}\right\}=\left\{X \in \mathcal{M} \underset{\alpha}{\underset{\alpha}{\rtimes}} G ; \bar{X} \eta \pi_{\alpha}\left(\left(\mathcal{M}_{w}^{\prime}\right)^{\prime}\right)\right\} .
$$

Proof. Take an arbitrary $X \in \mathcal{M} \stackrel{G W^{*}}{\rtimes} G$. Let $\bar{X}=U|\bar{X}|$ be the polar decomposition of $\bar{X}$ and $|\bar{X}|=\int_{0}^{\infty} \lambda d E(\lambda)$ the spectral resolution of $|\bar{X}|$. Put $X_{n}=\bar{X} E(n), n \in \mathbb{N}$. Suppose $\bar{X} \eta \pi_{\alpha}\left(\left(\mathcal{M}_{w}^{\prime}\right)^{\prime}\right)$. Then $E(n), X_{n} \in \pi_{\alpha}\left(\left(\mathcal{M}_{w}^{\prime}\right)^{\prime}\right)$, for every $n \in \mathbb{N}$. Moreover, from [32, Proposition I.4.12] it follows that for all $p \in \widehat{G}$, $\widehat{\alpha}_{p}(E(n))=E(n)$ and $\widehat{\alpha}_{p}\left(X_{n}\right)=X_{n}$, for all $n \in \mathbb{N}$. On the other hand, since,

$$
\widehat{\alpha}_{p}\left(X_{n}\right)=X_{n} \longrightarrow X, \text { strongly on } D(\mathcal{M}, G, \alpha) \text { and }
$$

$\widehat{\alpha}_{p}\left(X_{n}\right)=\overline{\widehat{\alpha}_{p}(X)} \widehat{\alpha}_{p}(E(n))=\overline{\widehat{\alpha}_{p}(X)} E(n) \longrightarrow \widehat{\alpha}_{p}(X)$, strongly on $D(\mathcal{M}, G, \alpha)$,

we have $\widehat{\alpha}_{p}(X)=X$. Conversely, suppose $\widehat{\alpha}_{p}(X)=X$, for every $p \in \widehat{G}$. Then in the same way as in the proof of Proposition 3.6, it is shown that $\widehat{\alpha}_{p}\left(X_{n}\right)=X_{n}$, for all $n \in \mathbb{N}$, which by [32, Proposition I.4.12] implies that $X_{n} \in \pi_{\alpha}\left(\left(\mathcal{M}_{w}^{\prime}\right)^{\prime}\right)$, for all $n \in \mathbb{N}$ and the respective sequence converges strongly to $X$. Hence, $\bar{X} \eta \pi_{\alpha}\left(\left(\mathcal{M}_{w}^{\prime}\right)^{\prime}\right)$. This completes the proof.

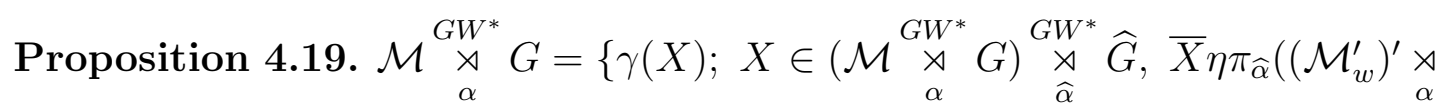
$G)\}$.

Proof. Using Proposition 3.6, Theorem 4.16 and Lemma 4.18, we obtain

$$
\begin{aligned}
& \mathcal{M} \underset{\alpha}{G W^{*}} G=\left\{Y \in\left(\mathcal{M}_{w}^{\prime}\right)^{\prime} \underset{D(\mathcal{M}, G, \alpha)}{\stackrel{G W^{*}}{\otimes}} \mathcal{B}\left(L^{2}(G)\right) ; \theta_{s}(Y)=Y, \forall s \in G\right\}
\end{aligned}
$$

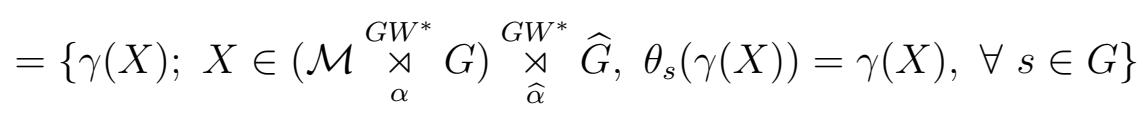

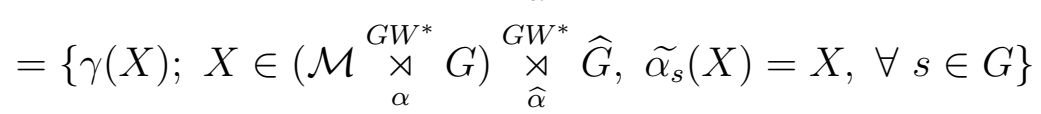

$$
\begin{aligned}
& =\left\{\gamma(X) ; X \in\left(\mathcal{M} \underset{\alpha}{G W^{*}} G\right) \underset{\widehat{\alpha}}{G W^{*}} \widehat{G}, \bar{X} \eta \pi_{\widehat{\alpha}}\left(\left(\mathcal{M}_{w}^{\prime}\right)^{\prime} \underset{\alpha}{\rtimes} G\right)\right\} .
\end{aligned}
$$

Question: Suppose that $\mathcal{M}$ is a $G W^{*}$-algebra on $\mathcal{D}$ in $\mathcal{H}$. Does the following equality

$$
\left\{X \in \mathcal{M} \underset{\alpha}{G W^{*}} G ; \bar{X} \eta \pi_{\widehat{\alpha}}\left(\left(\mathcal{M}_{w}^{\prime}\right)^{\prime}\right)\right\}=\pi_{\alpha}(\mathcal{M})
$$

hold? In the case of a positive answer, Proposition 4.19 can be clearly stated as

$$
\mathcal{M} \underset{\alpha}{G W^{*}} G=\gamma\left(\pi_{\widehat{\alpha}}\left(\mathcal{M} \underset{\alpha}{\stackrel{G W^{*}}{\rtimes}} G\right)\right)
$$




\section{Crossed products With modular aCtions}

Let $\mathcal{M}$ be a closed $O^{*}$-algebra on $\mathcal{D}$ in $\mathcal{H}$ such that $\mathcal{M}_{w}^{\prime} \mathcal{D} \subset \mathcal{D}$.

Definition 5.1. An element $\xi_{0}$ of $\mathcal{D}$ is said to be a standard vector for $\mathcal{M}$ if the following statements $(1)-(4)$ hold:

(1) $\mathcal{M} \xi_{0}$ is dense in $\mathcal{H}$.

(2) $\mathcal{M}_{w}^{\prime} \xi_{0}$ is dense in $\mathcal{H}$.

(3) $\Delta_{\xi_{0}}^{i t} \mathcal{D} \subset \mathcal{D}, \forall t \in \mathbb{R}$, where $\Delta_{\xi_{0}}$ is the modular operator of the achieved left Hilbert algebra $\left(\mathcal{M}_{w}^{\prime}\right)^{\prime} \xi_{0}$.

(4) $\Delta_{\xi_{0}}^{i t} \mathcal{M} \Delta_{\xi_{0}}^{-i t}=\mathcal{M}, \quad \forall t \in \mathbb{R}$.

Let $\xi_{0}$ be a standard vector for $\mathcal{M}$. We put

$$
\sigma_{t}^{\xi_{0}}(x)=\Delta_{\xi_{0}}^{i t} x \Delta_{\xi_{0}}^{-i t}, \quad x \in \mathcal{M}, \quad t \in \mathbb{R}
$$

Then $\sigma^{\xi_{0}}:=\left\{\sigma_{t}^{\xi_{0}}\right\}_{t \in \mathbb{R}}$ is a 1-parameter group of $*$-automorphisms of $\mathcal{M}$ and it is called the modular action associated with $\xi_{0}$. Furthermore, since $\mathcal{M}_{w}^{\prime} \xi_{0} \subset \mathcal{C}_{\sigma_{0}}(\mathcal{D})$ and it is dense in $\mathcal{H}$, it follows from Lemma 2.2 that $\left(\mathcal{M}, \mathbb{R}, \sigma^{\xi_{0}}\right)$ is an $O^{*}$-covariant system. Hence, as seen in Sections 3, 4, we may consider the crossed products $\mathcal{M} \underset{\sigma_{\xi_{0}}}{\stackrel{O^{*}}{\rtimes}} \mathbb{R}$ and $\mathcal{M} \underset{\sigma^{\xi_{0}}}{\stackrel{G W^{*}}{\rtimes}} \mathbb{R}$. To show that $\mathcal{M} \underset{\sigma^{\xi_{0}}}{\stackrel{O^{*}}{\rtimes}} \mathbb{R}$ and $\mathcal{M} \underset{\sigma^{\xi_{0}}}{\stackrel{G W^{*}}{\rtimes}} \mathbb{R}$ are independent of standard vectors $\xi_{0}$ up to isomorphism in case that $\mathcal{M}$ is a $G W^{*}$-algebra, we introduce the generalized Connes cocycle theorem [13, Theorem 2.5.6]

Lemma 5.2. Let $\xi_{0}$ and $\eta_{0}$ be standard vectors for a $G W^{*}$-algebra $\mathcal{M}$ on $\mathcal{D}$ in $\mathcal{H}$. Then there uniquely exists a strongly continuous map $\mathbb{R} \ni t \longmapsto u_{t} \in \mathcal{M}$ such that

(1) $\bar{u}_{t}$ is unitary, $t \in \mathbb{R}$;

(2) $\sigma_{t}^{\eta_{0}}(x)=u_{t} \sigma_{t}^{\xi_{0}}(x) u_{t}^{\dagger}, \quad t \in \mathbb{R}, \quad x \in \mathcal{M}$;

(3) $u_{s+t}=u_{t} \sigma_{t}^{\xi_{0}}\left(u_{s}\right)=\sigma_{t}^{\eta_{0}}\left(u_{s}\right) u_{t}, \quad t, s \in \mathbb{R}$.

Proposition 5.3. Let $\xi_{0}$ and $\eta_{0}$ be standard vectors for a $G W^{*}$-algebra $\mathcal{M}$ on

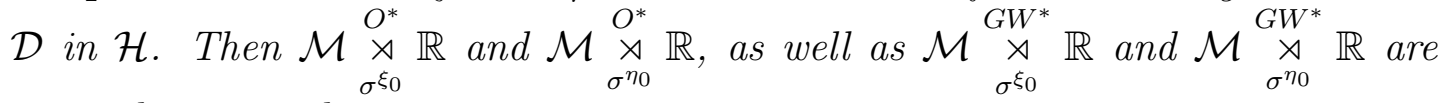
unitarily isomorphic.

Proof. We define a unitary operator $U$ on $L^{2}(\mathbb{R}, \mathcal{H})$ by

$$
(U \xi)(t):=u_{-t} \xi(t), \quad t \in \mathbb{R}, \quad \xi \in L^{2}(\mathbb{R}, \mathcal{H})
$$

Then

$$
U D_{0}\left(\mathcal{M}, \mathbb{R}, \sigma^{\xi_{0}}\right)=D_{0}\left(\mathcal{M}, \mathbb{R}, \sigma^{\eta_{0}}\right) .
$$

Indeed, take an arbitrary $\xi \in D_{0}\left(\mathcal{M}, \mathbb{R}, \sigma^{\xi_{0}}\right)$. Then $(U \xi)(t)=u_{-t} \xi(t) \in \mathcal{D}$, for every $t \in \mathbb{R}$ and the support of $U \xi$ is compact. Furthermore, since

$$
\sigma_{-t}^{\eta_{0}}(x)(U \xi)(t)=\sigma_{-t}^{\eta_{0}}(x) u_{-t} \xi(t)=(\text { by Lemma } 5.2(2)) u_{-t} \sigma_{-t}^{\xi_{0}}(x) \xi(t),
$$

for each $x \in \mathcal{M}$ and $t \in \mathbb{R}$, it follows that $\mathbb{R} \ni t \longmapsto \sigma_{-t}^{\eta_{0}}(x)(U \xi)(t) \in \mathcal{H}$ is continuous, which implies that $U \xi \in D_{0}\left(\mathcal{M}, \mathbb{R}, \sigma^{\eta_{0}}\right)$. Hence, $U D_{0}\left(\mathcal{M}, \mathbb{R}, \sigma^{\xi_{0}}\right) \subset$ 
$D_{0}\left(\mathcal{M}, \mathbb{R}, \sigma^{\eta_{0}}\right)$. Take an arbitrary $\eta \in D_{0}\left(\mathcal{M}, \mathbb{R}, \sigma^{\eta_{0}}\right)$. Then $\left(U^{*} \eta\right)(t)=u_{-t}^{\dagger} \eta(t) \in$ $\mathcal{D}$, for every $t \in \mathbb{R}$ and the support of $U^{*} \eta$ is compact. Since,

$$
\sigma_{-t}^{\xi_{0}}(x)\left(U^{*} \eta\right)(t)=\sigma_{-t}^{\xi_{0}}(x) u_{-t}^{\dagger} \eta(t)=(\text { by Lemma } 5.2(2)) u_{-t}^{\dagger} \sigma_{-t}^{\eta_{0}}(x) \eta(t),
$$

for each $x \in \mathcal{M}$ and $t \in \mathbb{R}$, it follows that $\mathbb{R} \ni t \longmapsto \sigma_{-t}^{\xi_{0}}(x)\left(U^{*} \eta\right)(t) \in \mathcal{H}$ is continuous which implies $U^{*} \eta \in D_{0}\left(\mathcal{M}, \mathbb{R}, \sigma^{\xi_{0}}\right)$. Hence, $U^{*} D_{0}\left(\mathcal{M}, \mathbb{R}, \sigma^{\eta_{0}}\right) \subset$ $D_{0}\left(\mathcal{M}, \mathbb{R}, \sigma^{\xi_{0}}\right)$. Thus, (5.1) holds. We next show that

$$
U^{*} \pi_{\sigma^{\eta_{0}}}(x) U \xi=\pi_{\sigma^{\xi_{0}}}(x) \xi, \quad x \in \mathcal{M}, \quad \xi \in D_{0}\left(\mathcal{M}, \mathbb{R}, \sigma^{\xi_{0}}\right) .
$$

Indeed, this follows from $\left(U^{*} \pi_{\sigma^{\eta_{0}}}(x) U \xi\right)(t)=u_{-t}^{\dagger} \sigma_{-t}^{\eta_{0}}(x) u_{-t} \xi(t)=($ by Lemma 5.2 (2)) $\sigma_{-t}^{\xi_{0}}(x) \xi(t)=\left(\pi_{\sigma^{\xi_{0}}}(x) \xi\right)(t)$, for each $x \in \mathcal{M}, \xi \in D_{0}\left(\mathcal{M}, \mathbb{R}, \sigma^{\xi_{0}}\right)$ and $t \in \mathbb{R}$. We shall also prove that

$$
\begin{gathered}
\left(U^{*} \lambda(t) U\right) \xi=\left(\pi_{\sigma^{\xi_{0}}}\left(u_{t}\right) \lambda(t)\right) \xi, \forall t \in \mathbb{R}, \forall \xi \in D_{0}\left(\mathcal{M}, \mathbb{R}, \sigma^{\xi_{0}}\right), \\
\left(U \lambda(t) U^{*}\right) \eta=\left(\pi_{\sigma^{\eta_{0}}}\left(u_{-t}\right) \lambda(t)\right) \eta, \forall t \in \mathbb{R}, \forall \eta \in D_{0}\left(\mathcal{M}, \mathbb{R}, \sigma^{\eta_{0}}\right) .
\end{gathered}
$$

Indeed, (5.3) follows from

$$
\begin{aligned}
\left(\left(U^{*} \lambda(t) U\right) \xi\right)(s) & =u_{-s}^{\dagger} \lambda(t)(U \xi)(s)=u_{-s}^{\dagger}(U \xi)(s-t) \\
& =u_{-s}^{\dagger} u_{t-s} \xi(s-t)=(\text { by Lemma } 5.2(3)) \sigma_{-s}^{\xi_{0}}\left(u_{t}\right)(\lambda(t) \xi)(s) \\
& =\left(\pi_{\sigma \xi_{0}}\left(u_{t}\right) \lambda(t) \xi\right)(s),
\end{aligned}
$$

for all $\xi \in D_{0}\left(\mathcal{M}, \mathbb{R}, \sigma^{\xi_{0}}\right)$ and $s, t \in \mathbb{R}$. The statement (5.4) is similarly shown.

By $(5.1)-(5.4)$, we have $U^{*}\left(\mathcal{M} \underset{\sigma^{\eta_{0}}}{\stackrel{O^{*}}{\rtimes}} \mathbb{R}\right) U=\mathcal{M} \underset{\sigma^{\xi_{0}}}{\underset{O^{*}}{\rtimes}} \mathbb{R}$. Finally, we show that

$$
U^{*}\left(\mathcal{M} \underset{\sigma^{\eta_{0}}}{\stackrel{G W^{*}}{\rtimes}} \mathbb{R}\right) U=\mathcal{M} \underset{\sigma^{\xi_{0}}}{\underset{\rtimes}{\triangleleft}} \mathbb{R} \text {. }
$$

Indeed, by [32, Theorem II.2.3], $U^{*}\left(\left(\mathcal{M}_{w}^{\prime}\right)^{\prime} \underset{\sigma^{\eta_{0}}}{\rtimes} \mathbb{R}\right) U=\left(\mathcal{M}_{w}^{\prime}\right)^{\prime} \underset{\sigma_{\xi_{0}}}{\rtimes} \mathbb{R}$. Hence,

$$
\begin{aligned}
& U^{*}\left(\mathcal{M} \underset{\sigma^{\eta_{0}}}{\stackrel{G W^{*}}{\rtimes}} \mathbb{R}\right) U=\left\{U^{*} X U ; \quad X \in \mathcal{M} \underset{\sigma^{\eta_{0}}}{\stackrel{G W^{*}}{\rtimes}} \mathbb{R}\right\} \\
& =\left\{U^{*} X U \in \mathcal{L}^{\dagger}\left(D\left(\mathcal{M}, \mathbb{R}, \sigma^{\xi_{0}}\right)\right) ; U^{*} X U \eta\left(\mathcal{M}_{w}^{\prime}\right)^{\prime} \underset{\sigma^{\xi_{0}}}{\rtimes} \mathbb{R}\right\} \\
& =\mathcal{M} \underset{\sigma \xi_{0}}{\stackrel{G W^{*}}{\rtimes}} \mathbb{R}
\end{aligned}
$$

This completes the proof.

We identify $\widehat{\mathbb{R}}$ with $\mathbb{R}$ itself as usual by setting $<s, t>=e^{i t s}$. As seen in Section 4 , the dual action is defined by

$$
\widehat{\sigma_{t}^{\xi_{0}}}(X)=\left(\mathbb{I} \bar{\otimes} m_{t}\right) X\left(\mathbb{I} \bar{\otimes} m_{t}\right)^{*}, \quad X \in \mathcal{M} \underset{\sigma^{\xi_{0}}}{G W^{*}} \mathbb{R}
$$

where $\left(m_{t} f\right)(s)=e^{-i s t} f(s), \quad f \in \mathcal{C}_{c}(\mathbb{R})$. We show that, in case of $\mathcal{M} \underset{\sigma^{\xi_{0}}}{G W^{*}} \mathbb{R}$, $\left\{\widehat{\sigma_{t}^{\xi_{0}}}\right\}_{t \in \mathbb{R}}$ is independent of $\xi_{0}$ up to isomorphism. 
Proposition 5.4. Let $\xi_{0}$ and $\eta_{0}$ be standard vectors for a $G W^{*}$-algebra $\mathcal{M}$ on $\mathcal{D}$ in $\mathcal{H}$. Put

$$
\delta(X)=U^{*} X U, \quad X \in \mathcal{M} \underset{\sigma^{\eta_{0}}}{\stackrel{G W^{*}}{R}} \mathbb{R}
$$

where $U$ is as in Proposition 5.3. Then $\delta$ is a*-isomorphism of $\mathcal{M} \underset{\sigma^{\eta_{0}}}{\stackrel{G W^{*}}{\rtimes}} \mathbb{R}$ onto $\mathcal{M} \underset{\sigma^{\xi_{0}}}{\stackrel{G W^{*}}{\rtimes}} \mathbb{R}$ and

$$
\widehat{\sigma_{t}^{\xi_{0}}}(\delta(X))=\delta\left(\widehat{\sigma_{t}^{\eta_{0}}}(X)\right), \text { for all } X \in \mathcal{M} \underset{\sigma^{\eta_{0}}}{\stackrel{\forall W^{*}}{\rtimes}} \mathbb{R} \text { and } t \in \mathbb{R} .
$$

Proof. It is easily shown that $U$ and $\left(\mathbb{I} \bar{\otimes} m_{t}\right)$ commute. Hence, we have

$$
\begin{aligned}
\widehat{\sigma_{t}^{\xi_{0}}}(\delta(X))=\left(I \bar{\otimes} m_{t}\right) U^{*} X U\left(I \bar{\otimes} m_{t}\right)^{*} & =U^{*}\left(I \bar{\otimes} m_{t}\right) X\left(I \bar{\otimes} m_{t}\right)^{*} U \\
& =U^{*} \widehat{\sigma_{t}^{\eta_{0}}}(X) U=\delta\left(\widehat{\sigma_{t}^{\eta_{0}}}(X)\right),
\end{aligned}
$$

for each $X \in \mathcal{M} \underset{\sigma^{\eta_{0}}}{\stackrel{G W^{*}}{\rtimes}} \mathbb{R}$ and $t \in \mathbb{R}$.

By Propositions 5.3 and 5.4, $\mathcal{M} \underset{\sigma^{\xi_{0}}}{\underset{\rtimes}{\rtimes}} \mathbb{R}$ and $\left(\mathcal{M} \underset{\sigma^{*} \xi_{0}}{\underset{\rtimes}{\rtimes}} \mathbb{R}\right) \underset{\sigma^{*}}{\stackrel{G W^{*}}{\rtimes}} \mathbb{R}$ are independent of $\xi_{0}$, and so we simply denote $\sigma^{\xi_{0}}$ and $\widehat{\sigma^{\xi}}$ by $\sigma$ and $\widehat{\sigma}$, respectively.

For any compact subset $K$ of $\mathbb{R}$ we put

$$
\xi_{K}=\xi_{0} \otimes \mathfrak{F}^{*} f_{K} \text { and } P_{K}=\mathbb{I} \bar{\otimes} \mathfrak{F}^{*} M_{\mathcal{X}_{K}} \mathfrak{F}
$$

where $\mathfrak{F}$ is the Fourier transform in $L^{2}(\mathbb{R}), f_{K}(s):=\mathcal{X}_{K}(s) e^{s / 2}, s \in \mathbb{R}$ and $M_{\mathcal{X}_{K}} f=\mathcal{X}_{K} f, f \in L^{2}(\mathbb{R})$. Then we have the following

Lemma 5.5. $\xi_{K} \in D(\mathcal{M}, \mathbb{R}, \sigma)$ and $P_{K}$ is a projection in $\left(\mathcal{M}_{w}^{\prime}\right)^{\prime} \underset{\sigma}{\rtimes} \mathbb{R}$ such that $P_{K} \xi_{K}=\xi_{K}$ and $\left\{P_{K}\right\} \uparrow I$.

Proof. There exists a sequence $\left\{f_{n}\right\}$ in $\mathcal{C}_{c}(\mathbb{R})$ such that $\lim _{n \rightarrow \infty}\left\|f_{n}-\mathfrak{F}^{*} f_{K}\right\|=0$. Since, $\xi_{0} \otimes f_{n} \in \mathcal{C}_{\sigma}(\mathcal{D}) \otimes \mathcal{C}_{c}(\mathbb{R}) \subset D_{0}(\mathcal{M}, \mathbb{R}, \sigma)$, for every $n \in \mathbb{N}$ and

$$
\begin{aligned}
& \xi_{0} \otimes f_{n} \underset{n \rightarrow \infty}{\longrightarrow} \xi_{K}, \\
& \left\|\pi_{\sigma}(x)\left(\xi_{0} \otimes f_{n}\right)-\pi_{\sigma}(x)\left(\xi_{0} \otimes f_{m}\right)\right\|=\left\|x \xi_{0}\right\|\left\|f_{n}-f_{m}\right\|_{m, n \rightarrow \infty}^{\longrightarrow} 0,
\end{aligned}
$$

for each $x \in \mathcal{M}$, we have $\xi_{K} \in \bigcap_{x \in \mathcal{M}} \mathcal{D}\left(\overline{\pi_{\sigma}(x)}\right)=D(\mathcal{M}, \mathbb{R}, \sigma)$.

Since, $P_{K}=\mathbb{I} \bar{\otimes} \mathfrak{F}^{*} M_{\mathcal{X}_{K}} \mathfrak{F}$ belongs to the von Neumann algebra generated by $\{\lambda(s) ; s \in \mathbb{R}\}, P_{K}$ is a projection in $\left(\mathcal{M}_{w}^{\prime}\right)^{\prime} \underset{\sigma}{\rtimes} \mathbb{R}$. It is clear that $P_{K} \xi_{K}=\xi_{K}$ and $\left\{P_{K}\right\} \uparrow I$.

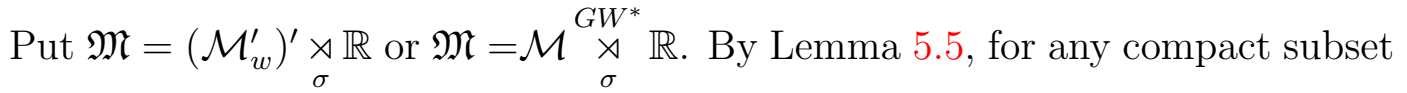
$K$ of $\mathbb{R}$ we can define a strongly positive linear functional $\tau_{K}$ on $\mathfrak{M}$ by

$$
\tau_{K}(X)=\left(X \xi_{K} \mid \xi_{K}\right), \quad X \in \mathfrak{M} .
$$

Then we have the following 
Lemma 5.6. Let $K$ and $K_{1}$ be compact subsets of $\mathbb{R}$ such that $K \subset K_{1}$. The following hold:

(1) $\left\|P_{K} X \xi_{K_{1}}\right\|=\left\|P_{K_{1}} X^{\dagger} \xi_{K}\right\|$, for all $X \in \mathfrak{M}$.

(2) $\tau_{K}(X) \leqq \tau_{K_{1}}(X)$, for all $X \in \mathfrak{M}_{+}$.

(3) $\tau_{K}\left(\widehat{\sigma}_{t}(X)\right)=e^{-t} \tau_{K+t}(X)$, for all $X \in \mathfrak{M}$ and $t \in \mathbb{R}$.

Proof. Take an arbitrary $X \in \mathcal{M} \underset{\sigma}{\underset{\rtimes}{\rtimes}} \mathbb{R}$. Then there exists a net $\left\{A_{\lambda}\right\}$ in $\left(\mathcal{M}_{w}^{\prime}\right)^{\prime} \underset{\sigma}{\rtimes}$ $\mathbb{R}$, which converges strongly* to $X$. The statement (1) follows from

$$
\begin{aligned}
\left\|P_{K} X \xi_{K_{1}}\right\|^{2} & =\lim _{\lambda}\left\|P_{K} A_{\lambda} \xi_{K_{1}}\right\|^{2} \\
& =\lim _{\lambda} \tau_{K_{1}}\left(\left(P_{K} A_{\lambda} P_{K_{1}}\right)^{*}\left(P_{K} A_{\lambda} P_{K_{1}}\right)\right) \\
& =\lim _{\lambda} \tau_{K_{1}}\left(\left(P_{K} A_{\lambda} P_{K_{1}}\right)\left(P_{K} A_{\lambda} P_{K_{1}}\right)^{*}\right) \\
& =\lim _{\lambda}\left\|P_{K_{1}} A_{\lambda}^{*} P_{K} \xi_{K_{1}}\right\|^{2} \\
& =\left\|P_{K_{1}} X^{\dagger} \xi_{K}\right\|^{2}
\end{aligned}
$$

(see also [32, Lemma II.3.4]). The statement (2) follows from

$$
\begin{aligned}
\tau_{K_{1}}(X)=\lim _{\lambda} \tau_{K_{1}}\left(A_{\lambda}\right) & =\lim _{\lambda}\left\{\tau_{K}\left(A_{\lambda}\right)+\left(A_{\lambda}\left(\xi_{K_{1}}-\xi_{K}\right) \mid \xi_{K !}-\xi_{K}\right)\right\} \\
& =\tau_{K}(X)+\left(X\left(\xi_{K_{1}}-\xi_{K}\right) \mid \xi_{K_{1}}-\xi_{K}\right)
\end{aligned}
$$

(see also [32, p. 48]). The statement (3) follows from

$$
\tau_{K}\left(\widehat{\sigma}_{t}(X)\right)=\lim _{\lambda} \tau_{K}\left(\widehat{\sigma}_{t}\left(A_{\lambda}\right)\right)=\lim _{\lambda} e^{-t} \tau_{K+t}\left(A_{\lambda}\right)=e^{-t} \tau_{K+t}(X)
$$

(see also [32, p. 49]).

By Lemma $5.6(2)$ we may define a map $\tau$ from $\mathfrak{M}_{+}$to $[0, \infty]$ by

$$
\tau(X)=\sup _{K} \tau_{K}(X), \quad X \in \mathfrak{M}_{+} .
$$

Then for the $W^{*}$-crossed product $\left(\mathcal{M}_{w}^{\prime}\right)^{\prime} \underset{\sigma}{\rtimes} \mathbb{R}$ we have

Lemma 5.7. ([32, Theorem II.3.5]) $\tau$ is a faithful semifinite normal trace on $\left(\left(\mathcal{M}_{w}^{\prime}\right)^{\prime} \underset{\sigma}{\rtimes} \mathbb{R}\right)_{+}$such that $\tau\left(\widehat{\sigma}_{t}(A)\right)=e^{-t} \tau(A)$, for each $A \in\left(\left(\mathcal{M}_{w}^{\prime}\right)^{\prime} \underset{\sigma}{\rtimes} \mathbb{R}\right)_{+}$and $t \in \mathbb{R}$.

On the other hand, for the $G W^{*}$-crossed product $\mathcal{M} \underset{\sigma}{G W^{*}} \mathbb{R}$ we obtain

Theorem 5.8. $\tau$ is a faithful normal trace on $\left(\mathcal{M} \underset{\sigma}{G W^{*}} \mathbb{R}\right)_{+}$such that $\tau\left(\widehat{\sigma}_{t}(X)\right)=$ $e^{-t} \tau(X)$, for all $X \in\left(\mathcal{M} \underset{\sigma}{G W^{*}} \mathbb{R}\right)_{+}$and $t \in \mathbb{R}$.

Proof. We first show that $\tau$ is a trace on $(\mathcal{M} \underset{\sigma}{\underset{\sim}{\rtimes}} \mathbb{R})_{+}$. Take arbitrary $X, Y \in$ $(\mathcal{M} \underset{\sigma}{\underset{\rtimes}{\rtimes}} \mathbb{R})_{+}$. For any compact $K$ in $\mathbb{R}$ we have

$$
\tau_{K}(X+Y)=\tau_{K}(X)+\tau_{K}(Y) \leqq \tau(X)+\tau(Y)
$$


and so $\tau(X+Y) \leqq \tau(X)+\tau(Y)$. Furthermore, for any compact $K_{1} \supset K$, we have by Lemma $5.6(2)$ that

$$
\tau_{K}(X)+\tau_{K_{1}}(Y) \leqq \tau_{K_{1}}(X)+\tau_{K_{1}}(Y)=\tau_{K_{1}}(X+Y) \leqq \tau(X+Y),
$$

which implies that $\tau(X)+\tau(Y) \leqq \tau(X+Y)$. Hence, $\tau(X+Y)=\tau(X)+\tau(Y)$. It is clear that $\tau(t X)=t \tau(X)$, for each $t \geq 0$.

We next show that $\tau\left(X^{\dagger} X\right)=\tau\left(X X^{\dagger}\right)$, for each $X \in \mathcal{M}_{\rtimes}^{G W^{*}} \mathbb{R}$. Indeed, take arbitrary compact subset $K$ of $\mathbb{R}$ and $X \in \mathcal{M} \underset{\sigma}{\underset{\sim}{\hookrightarrow W^{*}}} \mathbb{R}$. From Lemma 5.6 (1), we conclude that

$$
\begin{aligned}
\tau_{K}\left(X X^{\dagger}\right)=\left\|X^{\dagger} \xi_{K}\right\|^{2} & =\sup \left\{\left\|P_{K_{1}} X^{\dagger} \xi_{K}\right\|^{2} ; K_{1} \supset K\right\} \\
& =\sup \left\{\left\|P_{K} X \xi_{K_{1}}\right\|^{2} ; K_{1} \supset K\right\} \\
& \leqq \sup \left\{\left\|X \xi_{K_{1}}\right\|^{2} ; K_{1} \supset K\right\} \\
& =\sup \left\{\tau_{K_{1}}\left(X^{\dagger} X\right) ; K_{1} \supset K\right\} \\
& =\tau\left(X^{\dagger} X\right),
\end{aligned}
$$

where the suprema are taken over all compact sets $K_{1} \supset K$. And so $\tau\left(X X^{\dagger}\right) \leqq$ $\tau\left(X^{\dagger} X\right)$. Similarly, $\tau\left(X^{\dagger} X\right) \leqq \tau\left(X X^{\dagger}\right)$. Hence, $\tau\left(X^{\dagger} X\right)=\tau\left(X X^{\dagger}\right)$. Thus, $\tau$ is a trace on $\left(\mathcal{M} \underset{\sigma}{G W^{*}} \mathbb{R}\right)_{+}$.

Since, $\tau$ is the supremum of the normal functionals $\tau_{K}$, it is normal.

We show that $\tau$ is faithful. Indeed, suppose $\tau\left(X^{\dagger} X\right)=0$. Then we have

$$
X\left(a^{\prime} \xi_{0} \otimes \mathfrak{F}^{*} f_{K}\right)=\left(a^{\prime} \bar{\otimes} 1\right) X\left(\xi_{0} \otimes \mathfrak{F}^{*} f_{K}\right)=0,
$$

for each compact $K$ in $\mathbb{R}$ and $a^{\prime} \in \mathcal{M}_{w}^{\prime}$. Now, since $\left\{a^{\prime} \xi_{0} \otimes \mathfrak{F}^{*} f_{K} ; a^{\prime} \in \mathcal{M}_{w}^{\prime}\right.$, $K \subset \mathbb{R}$, compact $\}$ is dense in $L^{2}(\mathbb{R}, \mathcal{H})$, we have $X=0$. By Lemma 5.6 (3), $\tau\left(\widehat{\sigma_{t}}(X)\right)=e^{-t} \tau(X)$, for every $X \in\left(\mathcal{M} \underset{\sigma}{\underset{\sim}{G W^{*}}} \mathbb{R}\right)_{+}$and $t \in \mathbb{R}$. This completes the proof.

It is important to consider the following

Question. When is the trace $\tau$ on $(\mathcal{M} \underset{\sigma}{\underset{\sim}{\rtimes}} \mathbb{R})_{+}$semifinite? Concerning this question we present

Theorem 5.9. Suppose that the graph topologies $t_{\mathcal{M}}$ and $t_{\mathcal{M}^{\sigma}}$ on $\mathcal{D}$ coincide, where $\mathcal{M}^{\sigma}=\left\{x \in \mathcal{M} ; \sigma_{t}(x)=x, \forall t \in \mathbb{R}\right\}$ is the fixed point algebra for $\sigma$. Then $P_{K} \in \mathcal{M} \underset{\sigma}{\stackrel{G W^{*}}{\rtimes}} \mathbb{R}$, for each compact subset $K$ in $\mathbb{R}$. Moreover, $\tau$ is a faithful normal semifinite trace on $\left(\mathcal{M} \underset{\sigma}{G W^{*}} \mathbb{R}\right)_{+}$.

Proof. Since, $t_{\mathcal{M}}=t_{\mathcal{M}^{\sigma}}$ and the family of seminorms $\left\{\|\cdot\|_{x} ; x \in \mathcal{M}^{\sigma}\right\}$ is directed, for every $x \in \mathcal{M}$ there exists an element $x_{0}$ in $\mathcal{M}^{\sigma}$ such that

$$
\|x \xi\| \leqq\left\|x_{0} \xi\right\|, \forall \xi \in \mathcal{D} .
$$


We now show that $\sigma$ is strongly continuous. Indeed, for each $x \in \mathcal{M}, \xi \in \mathcal{D}$ and $t \in \mathbb{R}$, we have

$$
\begin{aligned}
\left\|\sigma_{t}(x) \xi-x \xi\right\| & \leqq\left\|\Delta^{i t} x \Delta^{-i t} \xi-\Delta^{i t} x \xi\right\|+\left\|\Delta^{i t} x \xi-x \xi\right\| \\
& =\left\|x \Delta^{-i t} \xi-x \Delta^{-i t} \Delta^{i t} \xi\right\|+\left\|\Delta^{i t} x \xi-x \xi\right\| \\
& \leqq(\text { by }(5.5))\left\|x_{0} \xi-x_{0} \Delta^{i t} \xi\right\|+\left\|\Delta^{i t} x \xi-x \xi\right\| \\
& =\left\|x_{0} \xi-\Delta^{i t} x_{0} \xi\right\|+\left\|\Delta^{i t} x \xi-x \xi\right\|,
\end{aligned}
$$

which implies that $\lim _{t \rightarrow 0}\left\|\sigma_{t}(x) \xi-x \xi\right\|=0$. Hence, from Proposition 2.9 it follows that

$\mathcal{D} \otimes \mathcal{C}_{c}(\mathbb{R})$ is dense in $D(\mathcal{M}, \mathbb{R}, \sigma)$ w.r.t. the graph topology $t \underset{\mathcal{M}}{\substack{G W_{\sigma}^{*} \\ \sigma}} \cdot$

We show now that $P_{K} D(\mathcal{M}, \mathbb{R}, \sigma) \subset D(\mathcal{M}, \mathbb{R}, \sigma)$. Take arbitrary $\xi \in \mathcal{D}$ and $f \in \mathcal{C}_{c}(\mathbb{R})$. Then there is a sequence $\left\{g_{n}\right\}$ in $\mathcal{C}_{c}(\mathbb{R})$ which converges to $\mathfrak{F}^{*} M_{\mathcal{X}_{K}} \mathfrak{F} f$ with respect to the $L^{2}$-norm. Since,

$$
\begin{aligned}
& \xi \otimes g_{n} \underset{n \rightarrow \infty}{\longrightarrow} P_{K}(\xi \otimes f) \text { and } \\
&\left\|\pi_{\sigma}(x)\left(\xi \otimes g_{n}\right)-\pi_{\sigma}(x)\left(\xi \otimes g_{m}\right)\right\|^{2}=\int\left\|x\left(g_{n}(s)-g_{m}(s)\right) \Delta^{i s} \xi\right\|^{2} d s \\
& \leqq(\text { by }(5.5)) \int\left\|x_{0}\left(g_{n}(s)-g_{m}(s)\right) \Delta^{i s} \xi\right\|^{2} d s \\
&=\int\left\|x_{0}\left(g_{n}(s)-g_{m}(s)\right) \xi\right\|^{2} d s \\
&=\left\|x_{0} \xi\right\|^{2}\left\|g_{n}-g_{m}\right\|_{2}^{2} \underset{n, m \rightarrow \infty}{\longrightarrow} 0, \forall x \in \mathcal{M},
\end{aligned}
$$

it follows from (5.6) that $P_{K}(\xi \otimes f) \in D(\mathcal{M}, G, \sigma)$, which implies

$$
P_{K}\left(\mathcal{D} \otimes \mathcal{C}_{c}(\mathbb{R})\right) \subset D(\mathcal{M}, \mathbb{R}, \sigma) .
$$

Furthermore, for every $x \in \mathcal{M}, \xi_{k} \in \mathcal{D}$ and $f_{k} \in \mathcal{C}_{c}(\mathbb{R})$, we have

$$
\begin{aligned}
\| \pi_{\sigma}(x) P_{K} & \left(\sum_{k} \xi_{k} \otimes f_{k}\right)\left\|^{2}=\right\| \sum_{k}\left(\mathfrak{F}^{*} M_{\mathcal{X}_{K}} \mathfrak{F} f_{k}\right)(s) \Delta^{-i s} x \Delta^{i s} \xi_{k} \|^{2} \\
& =\int\left\|x \sum_{k}\left(\mathfrak{F}^{*} M_{\mathcal{X}_{K}} \mathfrak{F} f_{k}\right)(s) \Delta^{i s} \xi_{k}\right\|^{2} d s \\
& \leqq(\operatorname{by}(5.5)) \int\left\|x_{0} \sum_{k}\left(\mathfrak{F}^{*} M_{\mathcal{X}_{K}} \mathfrak{F} f_{k}\right)(s) \xi_{k}\right\|^{2} d s \\
& =\int \sum_{k, j}\left(\left(\mathfrak{F}^{*} M_{\mathcal{X}_{K}} \mathfrak{F} f_{k}\right)(s) x_{0} \xi_{k} \mid\left(\mathfrak{F}^{*} M_{\mathcal{X}_{\mathcal{M}}} \mathfrak{F} f_{j}\right)(s) x_{0} \xi_{j}\right) d s \\
& =\sum_{k, j}\left(x_{0} \xi_{k} \mid x_{0} \xi_{j}\right)\left(\mathfrak{F}^{*} M_{\mathcal{X}_{K}} \mathfrak{F} f_{k} \mid \mathfrak{F}^{*} M_{\mathcal{X}_{K}} \mathfrak{F} f_{j}\right)
\end{aligned}
$$




$$
\begin{aligned}
& =\sum_{k, j}\left(x_{0} \xi_{k} \mid x_{0} \xi_{j}\right) \int_{K}\left(\mathfrak{F} f_{k}\right)(s) \overline{\left(\mathfrak{F} f_{j}\right)(s)} d s \\
& =\int_{K}\left\|\sum_{k}\left(\mathfrak{F} f_{k}\right)(s) x_{0} \xi_{k}\right\|^{2} d s \\
& \leqq \int\left\|\sum_{k}\left(\mathfrak{F} f_{k}\right)(s) x_{0} \xi_{k}\right\|^{2} d s \\
& =\sum_{k, j}\left(x_{0} \xi_{k} \mid x_{0} \xi_{j}\right)\left(\mathfrak{F} f_{k} \mid \mathfrak{F} f_{j}\right) \\
& =\sum_{k, j}\left(x_{0} \xi_{k} \mid x_{0} \xi_{j}\right)\left(f_{k} \mid f_{j}\right) \\
& =\left\|\sum_{k} x_{0} \xi_{k} \otimes f_{k}\right\|^{2} \\
& =\left\|\pi_{\sigma}\left(x_{0}\right)\left(\sum_{k} \xi_{k} \otimes f_{k}\right)\right\|^{2},
\end{aligned}
$$

which by (5.6) and (5.7) implies that $P_{K} D(\mathcal{M}, \mathbb{R}, \sigma) \subset D(\mathcal{M}, \mathbb{R}, \sigma)$ and by Lemma 5.5 that $P_{K} \in \mathcal{M} \underset{\sigma}{\stackrel{G W^{*}}{x}} \mathbb{R}$.

Now, according to Theorem 5.8, it remains only to show that $\tau$ is semifinite. We put

$$
\begin{aligned}
& \mathfrak{N}_{\tau}^{0}=\left\{X \in \mathcal{M} \underset{\sigma}{G W^{*}} \mathbb{R} ; \tau\left(X^{\dagger} X\right)<\infty\right\}, \\
& \mathfrak{N}_{\tau}=\left\{X \in \mathfrak{N}_{\tau}^{0} ; A X \in \mathfrak{N}_{\tau}^{0}, \forall A \in \mathcal{M} \underset{\sigma}{G W^{*}} \mathbb{R}\right\} .
\end{aligned}
$$

Then we have $P_{K} \in \mathfrak{N}_{\tau}$, for each compact subset $K$ of $\mathbb{R}$. Indeed, take any compact subset $K_{1}$ of $\mathbb{R}$ with $K_{1} \supset K$. Then by Lemma 5.6 (1), for any $A \in$ $\mathcal{M} \underset{\sigma}{\stackrel{G W^{*}}{\rtimes}} \mathbb{R}$, we obtain

$$
\tau_{K_{1}}\left(\left(P_{K} A\right)^{\dagger}\left(P_{K} A\right)\right)=\left\|P_{K} A \xi_{K_{1}}\right\|^{2}=\left\|P_{K_{1}} A^{\dagger} \xi_{K}\right\|^{2} .
$$

Hence, we conclude that

$$
\begin{aligned}
& \tau\left(\left(P_{K} A\right)^{\dagger}\left(P_{K} A\right)\right)=\left\|A^{\dagger} \xi_{K}\right\|^{2} \text { and } \\
& \tau\left(\left(P_{K} A\right)^{\dagger}\left(P_{K} A\right)\right)=\tau\left(\left(A P_{K}\right)\left(A P_{K}\right)^{\dagger}\right)=\left\|A \xi_{K}\right\|^{2},
\end{aligned}
$$

which yields $P_{K} \in \mathfrak{N}_{\tau}$. Furthermore, $P_{K} \uparrow I$ by Lemma 5.5. Hence, it follows that $\mathfrak{N}_{\tau}$ is strongly dense in $\mathcal{M} \underset{\sigma}{\underset{\rtimes}{\rtimes}} \mathbb{R}$, i.e., $\tau$ is semifinite. This completes the proof.

Corollary 5.10. Let $\mathcal{D}$ be a Fréchet space equipped with the graph topology $t_{\mathcal{M}^{\sigma}}$ defined by $\left\{\|\cdot\|_{x} ; x \in \mathcal{M}_{\sigma}\right\}$. In particular, let $\mathcal{D}=\bigcap_{n=1}^{\infty} \mathcal{D}\left(h^{n}\right)$, where $h$ is a positive self-adjoint operator such that $h \uparrow \mathcal{D} \in \mathcal{M}^{\sigma}$. Then $P_{K} \in \mathcal{M} \underset{\sigma}{\underset{x}{G}} \mathbb{R}$, 
for each compact subset $K$ of $\mathbb{R}$ and $\tau$ is a faithful normal semifinite trace on $\left(\mathcal{M} \underset{\sigma}{\stackrel{G W^{*}}{\rtimes}} \mathbb{R}\right)_{+}$

Proof. This is proved by applying the closed graph theorem and Theorem 5.9.

Example 5.11. Let $\left\{\xi_{n}\right\}_{n=0}^{\infty}$ be the orthonormal basis in the Hilbert space $L^{2}(\mathbb{R})$ consisting of all normalized Hermite functions. Let $q$ and $p$ be the position and the momentum operators on the Schwartz space $\mathcal{S}(\mathbb{R})$, respectively, and let $h$ be the number operator. That is,

$$
h=\frac{1}{2}(q+i p)(q-i p)=\sum_{n=0}^{\infty}(n+1) \xi_{n} \otimes \overline{\xi_{n}},
$$

where $\xi_{n} \otimes \overline{\xi_{n}}$ stands for the operator $\xi \longmapsto\left(\xi \mid \xi_{n}\right) \xi_{n}$. Then

$$
\mathcal{S}(\mathbb{R})=\bigcap_{n=0}^{\infty} \mathcal{D}\left(\bar{h}^{n}\right) .
$$

Let $\mathcal{A}$ be the $O^{*}$-algebra on $\mathcal{S}(\mathbb{R})$, generated by the position operator $q$, the momentum operator $p$, and all bounded functions of $h$.

Denote by $L^{2}(\mathbb{R}) \bar{\otimes} \overline{L^{2}(\mathbb{R})}$ the Hilbert space of all Hilbert-Schmidt operators on $L^{2}(\mathbb{R})$ with inner product $\langle x \mid y\rangle=\operatorname{tr}\left(y^{*} x\right), x, y \in L^{2}(\mathbb{R}) \bar{\otimes} \overline{L^{2}(\mathbb{R})}$. Put

$$
\begin{aligned}
\mathcal{S}(\mathbb{R}) \widetilde{\otimes} \overline{L^{2}(\mathbb{R})} & =\left\{x \in L^{2}(\mathbb{R}) \bar{\otimes} \overline{L^{2}(\mathbb{R})} ; x L^{2}(\mathbb{R}) \subset \mathcal{S}(\mathbb{R})\right\}, \\
\pi(a) x & =a x, \quad a \in \mathcal{A}, \quad x \in \mathcal{S}(\mathbb{R}) \widetilde{\otimes} \overline{L^{2}(\mathbb{R})} .
\end{aligned}
$$

Then $\pi$ is a self-adjoint $*$-representation of $\mathcal{A}$ on $\mathcal{S}(\mathbb{R}) \widetilde{\otimes} \overline{L^{2}(\mathbb{R})}$ such that

$$
\begin{gathered}
\pi(\mathcal{A})_{w}^{\prime}=\pi^{\prime}\left(\mathcal{B}\left(L^{2}(\mathbb{R})\right)\right):=\left\{\pi^{\prime}(a) ; a \in \mathcal{B}\left(L^{2}(\mathbb{R})\right)\right\}, \\
\left(\pi(\mathcal{A})_{w}^{\prime}\right)^{\prime}=\pi^{\prime \prime}\left(\mathcal{B}\left(L^{2}(\mathbb{R})\right)\right):=\left\{\pi^{\prime \prime}(a) ; \quad a \in \mathcal{B}\left(L^{2}(\mathbb{R})\right)\right\},
\end{gathered}
$$

where $\pi^{\prime}(a) x=x a, \pi^{\prime \prime}(a) x=a x, a \in \mathcal{B}\left(L^{2}(\mathbb{R})\right), x \in L^{2}(\mathbb{R}) \bar{\otimes} \overline{L^{2}(\mathbb{R})}$.

Now, for each $\beta>0$, put

$$
\Omega_{\beta}=\sum_{n=0}^{\infty} e^{-n \beta} \xi_{n} \bar{\otimes} \overline{\xi_{n}} .
$$

Then $\Omega_{\beta}$ is a standard vector for the self-adjoint $O^{*}$-algebra $\mathcal{M}:=\pi(\mathcal{A})$ on $\mathcal{S}(\mathbb{R}) \widetilde{\otimes} \overline{L^{2}(\mathbb{R})}$ and

$$
\sigma_{t}^{\Omega_{\beta}}(\pi(x))=\pi\left(\Omega_{\beta}^{i t} x \Omega_{\beta}^{-i t}\right), x \in \mathcal{A}, t \in \mathbb{R}
$$

(see [13, Example 2.4.24]). Furthermore, by (5.8) the essentially self-adjoint operator $\pi(h)$ is contained in $\mathcal{M}^{\sigma^{\Omega_{\beta}}}$ and by $(5.9), \mathcal{S}(\mathbb{R}) \widetilde{\otimes} \overline{L^{2}(\mathbb{R})}=\bigcap_{n=0}^{\infty} \mathcal{D}\left(\overline{\pi(h)}^{n}\right)$. Thus, $\left(\mathcal{M}, \Omega_{\beta}, \sigma^{\Omega_{\beta}}\right)$ satisfies the conditions of Corollary 5.10. Hence, $P_{K} \in \mathcal{M} \underset{\sigma^{\Omega_{\beta}}}{\stackrel{G W^{*}}{*}}$ $\mathbb{R}$, for each compact subset $K$ of $\mathbb{R}$ and $\tau$ is a faithful normal semifinite trace on $\left(\mathcal{M} \underset{\sigma^{\Omega}}{\stackrel{G W^{*}}{\rtimes}} \mathbb{R}\right)_{+}$ 
Also, for the maximal $O^{*}$-algebra $\mathcal{L}^{\dagger}(\mathcal{S}(\mathbb{R}))$ on $\mathcal{S}(\mathbb{R})$ instead of $\mathcal{A}$, the $*$-representation $\pi$, the standard vector $\Omega_{\beta}$ for the self-adjoint $O^{*}$-algebra $\pi\left(\mathcal{L}^{\dagger}(\mathcal{S}(\mathbb{R}))\right)$ on $\mathcal{S}(\mathbb{R}) \widetilde{\otimes} \overline{L^{2}(\mathbb{R})}$, and the modular automorphism group $\left\{\sigma_{t}^{\Omega_{\beta}}\right\}_{t \in \mathbb{R}}$ (for $\Omega_{\beta}$ ) may be defined similarly. Then it follows that $P_{K} \in \pi\left(\mathcal{L}^{\dagger}(\mathcal{S}(\mathbb{R}))\right) \underset{\sigma^{\Omega_{\beta}}}{\stackrel{G W^{*}}{\rtimes}} \mathbb{R}$, for each compact subset $K$ of $\mathbb{R}$ and that $\tau$ is a faithful normal semifinite trace on $\left(\pi\left(\mathcal{L}^{\dagger}(\mathcal{S}(\mathbb{R}))\right) \underset{\sigma^{\Omega_{\beta}}}{\stackrel{G W^{*}}{\rtimes}}\right.$ $\mathbb{R})_{+}$.

Finally we investigate the structure of type III-like $G W^{*}$-algebras. It is well known [29, Chapter XII, Theorem 1.1] that if the von Neumann algebra $\left(\mathcal{M}_{w}^{\prime}\right)^{\prime}$ is of type III, then $\left(\mathcal{M}_{w}^{\prime}\right)^{\prime} \rtimes \mathbb{R}$ is of type $\mathrm{II}_{\infty}$ and $\left(\mathcal{M}_{w}^{\prime}\right)^{\prime}$ is isomorphic to the crossed product $\left(\left(\mathcal{M}_{w}^{\prime}\right)^{\prime} \underset{\sigma}{x} \mathbb{R}\right) \underset{\sigma}{\rtimes} \underset{\mathbb{R}}{\sigma}$. We extend this result to the case of $G W^{*}$-algebras. Let $\mathcal{M}$ be a $G W^{*}$-algebra on $\mathcal{D}$ in $\mathcal{H}$. Suppose that the von Neumann algebra $\left(\mathcal{M}_{w}^{\prime}\right)^{\prime}$ is of type III. Then there exists a sequence $\left\{e_{n}\right\}, n \in \mathbb{N}$, of mutually orthogonal projections in $\left(\mathcal{M}_{w}^{\prime}\right)^{\prime}$, such that $e_{n} \sim \mathbb{I}$, for every $n \in \mathbb{N}$, and $\sum_{n=1}^{\infty} e_{n}=\mathbb{I I}[25$, Proposition 4.12]. Since, $e_{n} \sim \mathbb{I}$, for every $n \in \mathbb{N}$, there exists a sequence $\left\{v_{n}\right\}$, $n \in \mathbb{N}$, of partial isometries in $\left(\mathcal{M}_{w}^{\prime}\right)^{\prime}$, such that

$$
e_{n}=v_{n}^{*} v_{n} \text { and } \mathbb{I}=v_{n} v_{n}^{*}, \forall n \in \mathbb{N} .
$$

By [6, Theorem 5.3] we have the following

Lemma 5.12. Suppose that the graph topology $t_{\mathcal{M}}$ on $\mathcal{D}$ is defined by a sequence $\left\{\|\cdot\|_{t_{k}} ; t_{k} \in \mathcal{M}\right\}$ of seminorms such that $v_{n} \bar{t}_{k} \subset \bar{t}_{k} v_{n}$, for all $k, n \in \mathbb{N}$, where $\left\{v_{n}\right\}$ is as in (5.10). Then $\mathcal{M}$ is $*$-isomorphic to the $G W^{*}$-tensor product

$$
\mathcal{M}^{G W^{*}} \mathcal{B}\left(L^{2}(\mathbb{R})\right):=\left\{X \in \mathcal{L}^{\dagger}\left(\mathcal{D} \widetilde{\otimes} L^{2}(\mathbb{R})\right) ; \bar{X} \eta\left(\mathcal{M}_{w}^{\prime}\right)^{\prime}{ }^{W^{*}} \mathcal{B}\left(L^{2}(\mathbb{R})\right)\right\},
$$

where $\mathcal{D} \widetilde{\otimes} L^{2}(\mathbb{R}):=\bigcap_{x \in \mathcal{M}} \mathcal{D}(\overline{x \otimes 1})$.

Theorem 5.13. Let $\mathcal{M}$ be a $G W^{*}$-algebra on $\mathcal{D}$ in $\mathcal{H}$ with a standard vector $\xi_{0}$. Suppose that

(1) the von Neumann algebra $\left(\mathcal{M}_{w}^{\prime}\right)^{\prime}$ is of type III;

(2) $v_{n} \bar{t}_{k} \subset \bar{t}_{k} v_{n}$, for all $k, n \in \mathbb{N}$, where $\left\{v_{n}\right\}$ is as in (5.10) and $\left\{t_{k}\right\}$ is a sequence in $\mathcal{M}$ defining the graph topology $t_{\mathcal{M}}$.

Then $\left(\mathcal{M}_{w}^{\prime}\right)^{\prime} \rtimes \mathbb{R}$ is a von Neumann algebra of type $\mathrm{II}_{\infty}$ and $\mathcal{M}$ is isomorphic

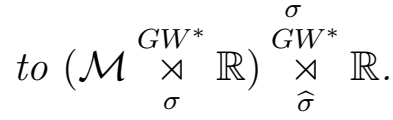

Proof. By Lemmas 4.5 and 4.6 we have

$$
W D(\mathcal{M}, \mathbb{R}, \sigma)=\bigcap_{x \in \mathcal{M}} \mathcal{D}(\overline{x \otimes 1})=\mathcal{D} \widetilde{\otimes} L^{2}(\mathbb{R}),
$$

where $W$ is the unitary operator on $L^{2}(\mathbb{R}, \mathcal{H})$ defined by

$$
(W \xi)(t)=\Delta^{i t} \xi(t), t \in \mathbb{R}, \xi \in L^{2}(\mathbb{R}, \mathcal{H}) .
$$


By Theorem 4.16 and Lemma 5.12, this implies

$$
\begin{aligned}
& \left(\mathcal{M} \underset{\sigma}{\stackrel{G W^{*}}{\rtimes}} \mathbb{R}\right) \underset{\widehat{\sigma}}{\stackrel{G W^{*}}{\rtimes}} \mathbb{R} \cong\left(\mathcal{M}_{w}^{\prime}\right)^{\prime} \underset{W D(\mathcal{M}, \mathbb{R}, \sigma)}{\stackrel{G W^{*}}{\otimes}} \mathcal{B}\left(L^{2}(\mathbb{R})\right) \\
& =\left\{X \in \mathcal{L}^{\dagger}(W D(\mathcal{M}, \mathbb{R}, \sigma)) ; \bar{X} \eta\left(\mathcal{M}_{w}^{\prime}\right)^{\prime}{ }^{W^{*}} \otimes \mathcal{B}\left(L^{2}(\mathbb{R})\right)\right\} \\
& =\left\{X \in \mathcal{L}^{\dagger}\left(\mathcal{D} \widetilde{\otimes} L^{2}(\mathbb{R})\right) ; \bar{X} \eta\left(\mathcal{M}_{w}^{\prime}\right)^{\prime}{ }^{W^{*}} \mathcal{B}\left(L^{2}(\mathbb{R})\right)\right\} \\
& =\mathcal{M}^{G W^{*}} \otimes \mathcal{B}\left(L^{2}(\mathbb{R})\right) \\
& \cong \mathcal{M} \text {. }
\end{aligned}
$$

This completes the proof.

Under the assumptions (1) and (2) of Theorem 5.13, $\left(\mathcal{M}_{w}^{\prime}\right)^{\prime}{ }_{\sigma} \mathbb{R}$ has a faithful normal semifinite trace, but we do not know whether the $G W^{*}$-algebra $\mathcal{M}_{\sigma}^{a W^{*}} \mathbb{R}$ has a faithful normal semifinite trace. By Theorems 5.9 and 5.13, we have the following

Theorem 5.14. Let $\mathcal{M}$ be a $G W^{*}$-algebra on $\mathcal{D}$ in $\mathcal{H}$ with a standard vector $\xi_{0}$. Suppose that

(1) the graph topologies $t_{\mathcal{M}}$ and $t_{\mathcal{M}^{\sigma}}$ coincide;

(2) the von Neumann algebra $\left(\mathcal{M}_{w}^{\prime}\right)^{\prime}$ is of type III;

(3) $v_{n} \overline{t_{k}} \subset \overline{t_{k}} v_{n}$, for all $n, k \in \mathbb{N}$, where $\left\{v_{n}\right\}$ is as in (5.10) and $\left\{t_{k}\right\}$ is a sequence in $\mathcal{M}$ defining the graph topology $t_{\mathcal{M}}$.

Then $\left(\mathcal{M}_{w}^{\prime}\right)^{\prime} \underset{\sigma}{\rtimes} \mathbb{R}$ is a von Neumann algebra of type $\mathrm{II}_{\infty}, \mathcal{M} \underset{\sigma}{G W^{*}} \mathbb{R}$ has a faithful

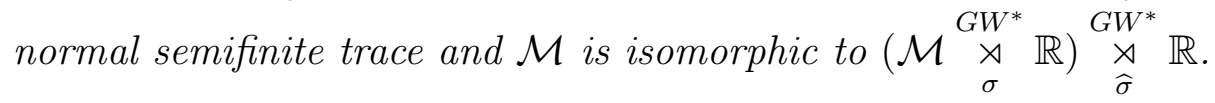

Remark 5.15. Under the assumptions of Theorem 5.14, we have

$$
\mathcal{D} \widetilde{\otimes} L^{2}(\mathbb{R})=W D(\mathcal{M}, \mathbb{R}, \sigma)=D(\mathcal{M}, \mathbb{R}, \sigma) .
$$

Indeed, take arbitrary $\xi \in \mathcal{D} \widetilde{\otimes} L^{2}(\mathbb{R})$ and $x \in \mathcal{M}$. Then there exist sequences $\left\{\xi_{k}\right\}$ and $\left\{f_{k}\right\}$ in $\mathcal{D}$ and $C_{c}(\mathbb{R})$ respectively, such that

$$
\sum_{k=1}^{n} \xi_{k} \otimes f_{k} \underset{n \rightarrow \infty}{\longrightarrow} \xi \text { and }\left(x_{0} \otimes 1\right)\left(\sum_{k=1}^{n} \xi_{k} \otimes f_{k}\right) \underset{n \rightarrow \infty}{\longrightarrow}\left(x_{0} \widetilde{\otimes} 1\right) \xi,
$$

where $x_{0}$ is as in (5.5); therefore,

$$
\begin{aligned}
\left\|\pi_{\sigma}(x) \sum_{k=n}^{m} \xi_{k} \otimes f_{k}\right\|^{2} & =\int\left\|x \Delta^{i s} \sum_{k=n}^{m} f_{k}(s) \xi_{k}\right\|^{2} d s \leqq \int\left\|x_{0} \sum_{k=n}^{m} f_{k}(s) \xi_{k}\right\|^{2} d s \\
& =\left\|\left(x_{0} \otimes 1\right)\left(\sum_{k=n}^{m} \xi_{k} \otimes f_{k}\right)\right\|_{n \rightarrow \infty}^{2} 0 .
\end{aligned}
$$

Hence, $\xi \in\left(\widetilde{\mathcal{Q} \widetilde{C_{c}}(\mathbb{R})}\right)^{t_{\pi_{\sigma}}}=D(\mathcal{M}, \mathbb{R}, \sigma)$ and $\mathcal{D} \widetilde{\otimes} L^{2}(\mathbb{R}) \subset D(\mathcal{M}, \mathbb{R}, \sigma)$, where the symbol $\left(\mathcal{D} \widetilde{\otimes C_{c}}(\mathbb{R})\right)^{t_{\pi_{\sigma}}}$ means completion with respect to the graph topology $t_{\pi_{\sigma}}$ on $\mathcal{D}\left(\pi_{\sigma}\right)$. The inverse inclusion $D(\mathcal{M}, \mathbb{R}, \sigma) \subset \mathcal{D} \widetilde{\otimes} L^{2}(\mathbb{R})$ follows from 
Theorem 5.14 (1) and the equality

$$
\left\|\pi_{\sigma}\left(x_{0}\right) \sum_{k=1}^{n} \xi_{k} \otimes f_{k}\right\|=\left\|\left(x_{0} \otimes 1\right) \sum_{k=1}^{n} \xi_{k} \otimes f_{k}\right\|,
$$

where $x_{0} \in \mathcal{M}^{\sigma}$ and $\sum_{k=1}^{n} \xi_{k} \otimes f_{k} \in \mathcal{D} \otimes C_{c}(\mathbb{R})$. Thus, we conclude that $D(\mathcal{M}, \mathbb{R}, \sigma)=\mathcal{D} \widetilde{\otimes} L^{2}(\mathbb{R})$.

By Theorems 5.13 and 5.14 , we now have the following

Corollary 5.16. Let $\mathcal{M}$ be a $G W^{*}$-algebra on $\mathcal{D}:=\bigcap_{n \in \mathbb{N}} \mathcal{D}\left(h^{n}\right)$ with a standard vector, where $h$ is a positive self-adjoint operator in $\mathcal{H}$ such that $h \uparrow_{\mathcal{D}} \in \mathcal{M}$. Suppose that $\left(\mathcal{M}_{w}^{\prime}\right)^{\prime}$ is a von Neumann algebra of type III and that $v_{n} h \subset h v_{n}$, for all $n \in \mathbb{N}$, where $\left\{v_{n}\right\}$ is a sequence of partial isometries in $\left(\mathcal{M}_{w}^{\prime}\right)^{\prime}$ satisfying (5.10). Then $\left(\mathcal{M}_{w}^{\prime}\right)^{\prime} \rtimes \mathbb{R}$ is a von Neumann algebra of type $\mathrm{II}_{\infty}$ and $\mathcal{M}$ is isomorphic to $\left(\mathcal{M} \underset{\sigma}{G W^{*}} \mathbb{R}\right) \underset{\widehat{\sigma}}{\stackrel{G W^{*}}{\rtimes}} \mathbb{R}$. Moreover, if $h \uparrow_{\mathcal{D}} \in \mathcal{M}^{\sigma}$, then $\mathcal{M} \underset{\sigma}{\stackrel{G W^{*}}{\rtimes}} \mathbb{R}$ has a faithful normal semifinite trace.

We now give an example of a $G W^{*}$-algebra satisfying the assumptions of Theorems 5.13 and 5.14 .

Example 5.17. Let $\mathcal{M}_{0}$ be a von Neumann algebra on $\mathcal{H}$ of type III with a cyclic and separating vector $\xi_{0}$. Let $\left\{e_{n}\right\}$ be a sequence of pairwise orthogonal projections in $\mathcal{M}_{0}$ such that $\sum_{n=1}^{\infty} e_{n}=\mathbb{I}$ and $e_{n} \sim \mathbb{I}$, for all $n \in \mathbb{N}$. Let $\left\{v_{n}\right\}$ be a sequence of partial isometries in $\mathcal{M}_{0}$ such that $e_{n}=v_{n}^{*} v_{n}$ and $\mathbb{I}=v_{n} v_{n}^{*}$, for all $n \in \mathbb{N}$. Suppose that $\left\{v_{n}\right\}^{\prime} \cap \mathcal{M}_{0}$ is a von Neumann algebra of infinite dimension. Then there exists a sequence $\left\{f_{n}\right\}$ of pairwise orthogonal projections in $\left\{v_{n}\right\}^{\prime} \cap \mathcal{M}_{0}$, such that $\sum_{n=1}^{\infty} f_{n}=\mathbb{I}$. Then since $\left\|\xi_{0}\right\|^{2}=\sum_{n=1}^{\infty}\left\|f_{n} \xi_{0}\right\|^{2}$, there exists a subsequence $\left\{f_{n_{k}}\right\}$ of $\left\{f_{n}\right\}$ such that $\left\|f_{n_{k}} \xi_{0}\right\| \leq e^{-k}$, for all $k \in \mathbb{N}$. We put

$$
\left\{\begin{aligned}
\mathcal{D}(h) & =\left\{\xi \in \mathcal{H} ; \sum_{k=1}^{\infty} k^{2}\left\|f_{n_{k}} \xi\right\|^{2}<\infty\right\} \\
h \xi & =\sum_{k=1}^{\infty} k f_{n_{k}} \xi, \xi \in \mathcal{D}(h) .
\end{aligned}\right.
$$

Then $h$ is a positive self-adjoint unbounded operator affiliated with $\left\{v_{n}\right\}^{\prime} \cap \mathcal{M}_{0}$. Furthermore, we define a $G W^{*}$-algebra $\mathcal{M}$ on $\mathcal{D}$ as follows:

$$
\left\{\begin{array}{l}
\mathcal{D}:=\mathcal{D}^{\infty}(h)=\left\{\xi \in \mathcal{H} ; \sum_{k=1}^{\infty} k^{l}\left\|f_{n_{k}} \xi\right\|^{2}<\infty, \forall l \in \mathbb{N}\right\} \\
\mathcal{M}:=\left\{x \in \mathcal{L}^{\dagger}(\mathcal{D}), \bar{x} \eta \mathcal{M}_{0}\right\} .
\end{array}\right.
$$

Then $\xi_{0} \in \mathcal{D}$ and $\mathcal{M}$ is a $G W^{*}$-algebra on $\mathcal{D}$ in $\mathcal{H}$ over $\mathcal{M}_{0}$ with standard vector $\xi_{0}$. Moreover, $v_{n} h^{m} \subset h^{m} v_{n}$, for all $n, m \in \mathbb{N}$. Thus, $\mathcal{M}$ satisfies all assumptions of Corollary 5.16. 
In case that $\left\{v_{n}\right\}^{\prime} \cap \mathcal{M}_{0}^{\sigma}$ is a von Neumann algebra of infinite dimension, we can similarly construct a positive self-adjoint unbounded operator affiliated with $\left\{v_{n}\right\}^{\prime} \cap \mathcal{M}_{0}^{\sigma}$ and a $G W^{*}$-algebra $\mathcal{M}$ on $\mathcal{D}^{\infty}(h)$ with a faithful normal semifinite trace such that $\mathcal{M}$ and $(\mathcal{M} \underset{\sigma}{\underset{\gamma}{\rtimes}} \mathbb{R}) \underset{\widehat{\sigma}}{\underset{W^{*}}{W^{*}}} \mathbb{R}$ are isomorphic.

Acknowledgements. The present work was performed at the Department of Mathematics, University of Athens, the Department of Applied Mathematics, University of Fukuoka and the Institute of Mathematics, Leipzig University. The authors thank the Departments that they have visited for the financial support and overwhelming hospitality. They also thank the referees for their comments.

\section{REFERENCES}

1. J.P. Antoine, A. Inoue H. Ogi and C. Trapani, Standard generalized vectors in the space of Hilbert-Schmidt operators, Ann. Inst. H. Poincaré Phys. Théor., 63 (1995), no. 2, 177-210.

2. J.P. Antoine, A. Inoue and C. Trapani, Partial $*$-Algebras and their Operator Realizations, Math. Appl. 553, Kluwer Academic, Dordrecht, 2002.

3. F. Bagarello, Algebras of unbounded operators and physical applications: a survey, Rev. Math. Phys. 19 (2007), no. 3, 231-272.

4. O. Bratelli and D.W. Robinson, Operator Algebras and Quantum Statistical Mechanics I and II, Springer Verlag, New York, Heidelberg, Berlin, 1979 and 1981.

5. A. Connes, Une classification des facteurs de type III, Ann. Sci. Èc. Norm. Supèr. (4) 6 (1973), 133-252.

6. M. Fragoulopoulou, A. Inoue and M. Weigt, Tensor products of unbounded operator algebras, Rocky Mountain J. Math. 44 (2014), no. 3, 895-912.

7. R. Haag, Local Quantum Physics, Springer-Verlag, Berlin, 1992.

8. A. Inoue, Unbounded genaralization of left Hilbert algebras, J. Funct. Anal. 34 (1979), no. 3, 339-362.

9. A. Inoue, Unbounded genaralization of left Hilbert algebras II, J. Funct. Anal. 35 (1980), no. 3, 230-250.

10. A. Inoue, Modular structure of algebras of unbounded operators, Math. Proc. Cambridge Philos. Soc. 111 (1992), no. 2, 369-386.

11. A. Inoue, Modular systems induced by trace functionals on algebras of unbounded operators, J. Math. Phys. 35 (1994), no. 1, 435-442.

12. A. Inoue, Standard systems for semifinite $O^{*}$-algebras, Proc. Amer. Math. Soc. 125 (1997), no. 11, 3303-3312.

13. A. Inoue, Tomita-Takesaki Theory in Algebras of Unbounded Operators, Lecture Notes Math. 1699, Springer-Verlag, 1998.

14. M. Joiţa, Crossed Products of Locally $C^{*}$-Algebras, Editura Academiei Române, Bucureşti, 2007.

15. G. Karpilovsky, The Algebraic Structure of Crossed Products, North-Holland, Amsterdam, 1987.

16. G. Lassner, Algebras of unbounded operators and quantum dynamics, Phys. A 124 (1984), no. 1-3, 471-480.

17. D.S. Passman, Infinite Crossed Products, Academic Press, Boston, Vol. 135, 1989.

18. G.K. Pedersen, $C^{*}-$ Algebras and their Automorphism Groups, Academic Press, London, 1979.

19. S. Sakai, Operator Algebras in Dynamical Systems, Cambridge Univ. Press, Cambridge, 1991.

20. Yu. Savchuk and K. Schmüdgen, Unbounded induced representations of $*$-algebras, Algebr. Represent. Theory 16 (2013), no. 2, 309-376. 
21. K. Schmüdgen, Unbounded Operator Algebras and Representation Theory, BirkhäuserVerlag, Basel, 1990.

22. K. Schmüdgen and E. Wagner, Hilbert space representations of cross product algebras, J. Funct. Anal. 200 (2003), no. 2, 451-493.

23. K. Schmüdgen and E. Wagner, Hilbert space representations of cross product algebras. II, Algebr. Represent. Theory 9 (2006), no. 5, 431-464.

24. S. Strătilă, Modular Theory in Operator Algebras, Abacus Press, Tunbridge Wells, England, 1981.

25. S. Strătilă, L. Zsidó, Lectures on von Naumann Algebras, Abacus Press, Tunbridge Wells, England, 1979.

26. S.J. Summers, Tomita-Takesaki Modular Theory, Encyclopedia Math. Phys., edited by J.-P. Francoise, G. Naber and T.S. Tsun, (Elsevier), 5 (2006), 251-257.

27. M. Takesaki, Tomita's theory of modular Hilbert algebras and its applications, Lecture Notes in Mathematics 128, Springer-Verlag, 1970.

28. M. Takesaki, Duality for crossed products and the structure of von Neumann algebras of type III, Acta Math. 131 (1973), 249-310.

29. M. Takesaki, Theory of Operator Algebras II, Springer-Verlag, New York-HeidelbergBerlin, 2003.

30. W. Thirring, A Course in Mathematical Physics 4: Quantum Mechanics of Large Systems, Springer-Verlag, Wien, 1983.

31. T. Turumaru, Crossed products of operator algebras, Tôhoku Math. J. (2) 10 (1958), 355365.

32. A. Van Daele, Continuous crossed products and Type III von Neumann algebras, Cambridge Univ. Press, Cambridge, 1978.

33. D.P. William, Crossed products of $C^{*}$-algebras, Mathematical Surveys and Monographs, Vol. 134, 2007.

34. G. Zeller-Meier, Produits croisés d'une $C^{*}$-algèbre par une groupe d'automorphismes, J. Math. Pures Appl. (9) 47 (1968), 101-239.

${ }^{1}$ Department of Mathematics, University of Athens, Panepistimiopolis, Athens 15784, GREECE.

E-mail address: fragoulop@math.uoa.gr.

2 Department of Applied Mathematics, University of Fukuoka, Fukuoka 8140180, JAPAN.

E-mail address: a-inoue@fukuoka-u.ac.jp

3 Mathematisches Institut, Universität Leipzig, Postfach 1009 20, D-04009 LEIPZig, GERMANY.

E-mail address: kuersten@math.uni-leipzig.de 